NASA Contractor Report 4096

\title{
An Analysis of the Crossover Between Local and Massive Separation on Airfoils
}

M. Barnett and J. E. Carter

CONTRACT NAS1-16585

SEPTEMBER 1987

\section{N/SN}


NASA Contractor Report 4096

\section{An Analysis of the Crossover Between Local and Massive Separation on Airfoils}

M. Barnett and J. E. Carter

United Technologies Research Center

East Hartford, Connecticut

Prepared for

Langley Research Center

under Contract NAS1-16585

\section{Nush}

National Aeronautics

and Space Administration

Scientific and Technical Information Office 


\section{TABLE OF CONTENTS}

SUMMARY . . . . . . . . . . . . . . . . . . . . 1

ACKNOWLEDGMENTS . . . . . . . . . . . . . . . . . . . . 2

LIST OF SYMBOLS . . . . . . . . . . . . . . . . . . . . . . . 3

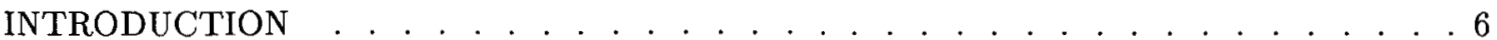

Theoretical Basis for a Model of Stall . . . . . . . . . . . . . . . . . . . . . . 7

PROBLEM DESCRIPTION AND FORMULATION . . . . . . . . . . . . . . . . . 14

Triple-Deck Theory . . . . . . . . . . . . . . . . . . . . . . . . . . . . . 14

Interacting Boundary-Layer Theory . . . . . . . . . . . . . . . . . . . . 17

NUMERICAL METHOD . . . . . . . . . . . . . . . . . . . . 23

Viscous Equations . . . . . . . . . . . . . . . . . . . . . 23

Inviscid Equations . . . . . . . . . . . . . . . . . . . . 26

Interacting Boundary-Layer Equations . . . . . . . . . . . . . . . . . . . 29

DISCUSSION OF RESULTS . . . . . . . . . . . . . . . . . . . . . . . . . . 31

Triple-Deck Analysis . . . . . . . . . . . . . . . . . . . . . . . . . . . . . 31

Interacting Boundary-Layer Analysis . . . . . . . . . . . . . . . . . 32

'Turbulence Effects . . . . . . . . . . . . . . . . . . . . . 37

Boundary-Layer Control . . . . . . . . . . . . . . . . . . . . 39

CONCLUDING REMARKS . . . . . . . . . . . . . . . . . . . . . 44

Future Directions . . . . . . . . . . . . . . . . . . . . . . . 45

REFERENCES .......................... . . . . . 46

APPENDIX A: SOLUTION OF THE TRIPLE-DECK PROBLEM . . . . . . . . . . . 49

APPENDIX B: TURBULENCE MODEL . . . . . . . . . . . . . . . . . . . . . . 51

APPENDIX C: BOUNDARY-LAYER EQUATION COEFFICIENTS . . . . . . . . . . 53

APPENDIX D: BOUNDARY-LAYER EQUATION SOLUTION . . . . . . . . . . . . . 54

APPENDIX E: INVISCID INTEGRAL REPRESENTATION COEFFICIENTS . . . . . 57

FIGURES . . . . . . . . . . . . . . . . . . . . . . . . . 61 


\section{SUMMARY}

Massive separation on airfoils operating at high Reynolds number is an important problem to the aerodynamicist, since its onset generally determines the limiting performance of an airfoil, and it can lead to serious problems related to aircraft control as well as turbomachinery operation. In the present study, the phenomenon of crossover between local separation and massive separation on realistic airfoil geometries induced by airfoil thickness is investigated for low speed (incompressible) flow. The problem is studied both for the asymptotic limit of infinite Reynolds number using triple-deck theory, and for finite Reynolds number using interacting boundary-layer theory.

Numerical results are presented which follow the evolution of the flow as it develops from a mildly separated state to one dominated by the massively separated flow structure as the thickness of the airfoil geometry is systematically increased. The results of the triple-deck and the interacting boundary-layer analyses of the two airfoils which are considered are found to be in qualitative agreement.

The effect of turbulence upon the evolution of the flow is considered, and the impact is significant, with the principal effect being the suppression of the onset of separation. A turbulent massively separated solution is presented for flow past a circular cylinder, and the calculated surface pressure distribution is found to agree well with experimental data when the proper eddy pressure level is specified.

Finally, the effect of surface suction and injection for boundary-layer control is considered. Application of mild surface suction is shown to eliminate massive separation in cases which would otherwise be stalled, and tangential injection, represented here by a specified slip velocity along the surface, is shown to reduce or eliminate separation.

The approach which has been developed as part of the present effort shows that interacting boundarylayer theory provides a valuable tool for the analysis of boundary-layer separation up to and beyond stall. Another important conclusion of the present study is that interacting boundary-layer theory provides an efficient tool for the analysis of the effect of turbulence and boundary-layer control upon separated viscous flow. 


\section{ACKNOWLEDGEMENTS}

The authors would like to acknowledge the valuable contributions of Dr. M. J. Werle of UTRC and Profs. A. P. Rothmayer of Iowa State University and F. T. Smith of University College London to this study. In addition, the authors note that the circular cylinder calculation and the drag and boundary-layer control analyses were performed with the support of the United Technologies Corporation. 


\section{LIST OF SYMBOLS}

Latin

$a_{0}, a_{1}, \ldots a_{4} \quad$ Airfoil profile coefficients, Eq. (11)

$A, A^{+} \quad$ Coefficients in turbulence model, Eq. (B.2b)

$\bar{A}, \bar{B}, \ldots \bar{H} \quad$ Coefficients of discrete boundary-layer momentum equation, Eq. (45)

$A_{o}, B_{o}, \ldots E_{o} \quad$ Coefficients of discrete boundary-layer momentum equation at wake centerline, Eq. (47b)

$b \quad$ Coefficient of free streamline growth, Eq. (10)

$\widetilde{C}, \widetilde{D} \quad$ Coefficients in inviscid equation, Eq. (57c)

$C_{D} \quad$ Drag coefficient, Eq. (68)

$C_{f}, C_{p} \quad$ Skin friction and pressure coefficients

$C_{0}, C_{1} \quad$ Coefficient for displacement thickness growth, Eqs. (E.9b) and (E.14)

$D_{i j} \quad$ Influence coefficient for discretized inviscid equations, Eqs. (55b) and (60)

$f$

Airfoil shape function, Eq. (11), or distance from baseline to body or wake centerline, Fig. 3

F

Transformed boundary-layer tangential velocity, Eq. (20)

$\bar{g}$

Coefficient in inviscid equation, Eq. (56)

$h, \widetilde{h} \quad$ Unscaled, scaled airfoil thickness parameter

$i, j \quad$ Streamwise and normal mesh indices, Figs. 4 and 5

I Term in inviscid equation for massive separation model, Eq. (36c)

$I_{a}, I_{b} \quad$ Integrals in triple-deck analysis, Eqs. (A.3a) and (A3.b)

$L \quad$ Reference length

LSI Local strong-interaction

MS Massive separation

$n$

Scaled normal distance from baseline curve, Eq. (12b)

$\tilde{N}$

Pressure gradient term in turbulence model, Eq. (B.2c)

$p \quad$ Pressure

$P \quad$ Inviscid coefficient, Eq. (59b)

$P, Q \quad$ Coefficients of discrete boundary-layer continuity equation, Eq. (39) 


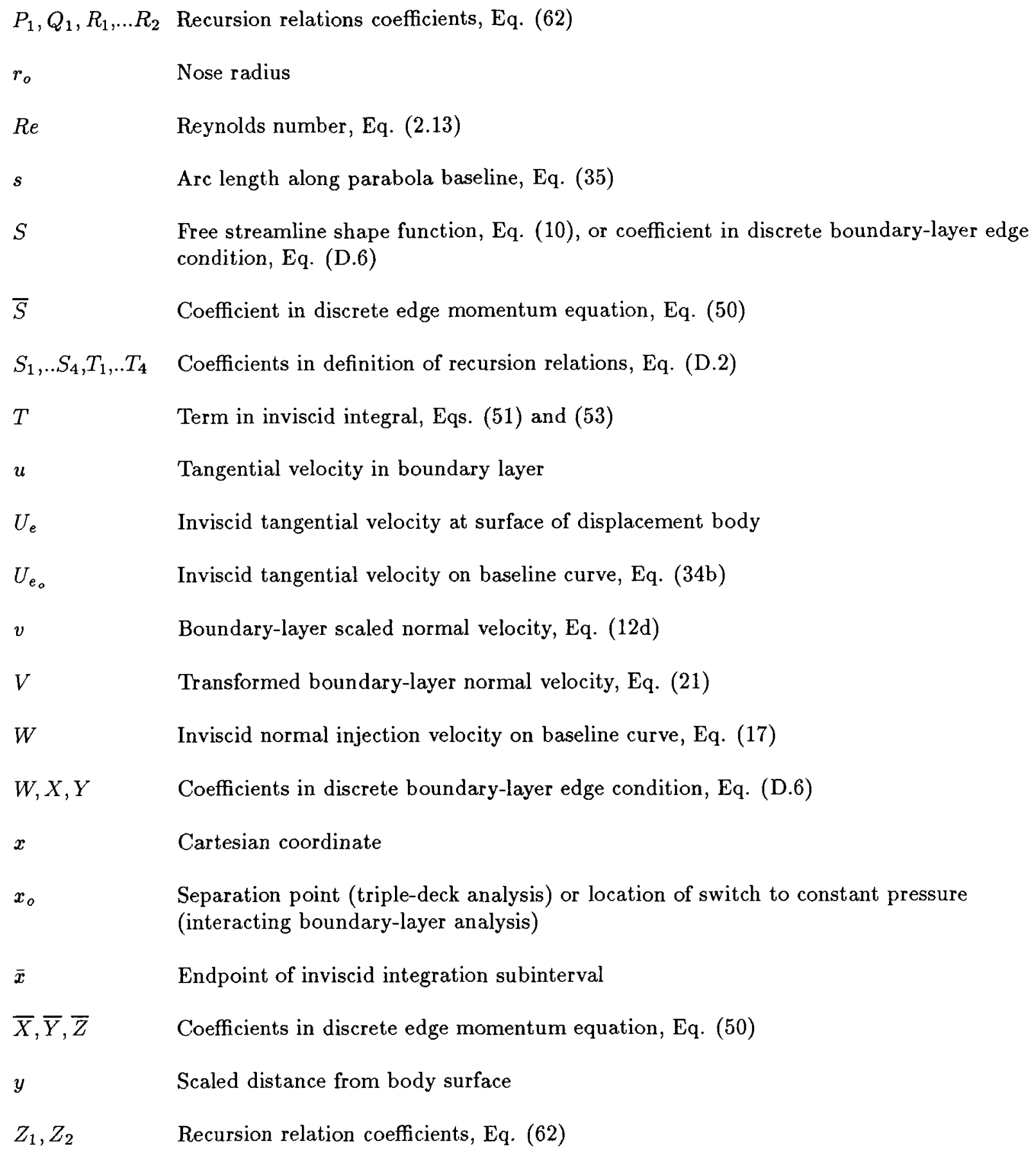




\section{Greek}

$\alpha$

$\beta_{\circ}$

$\beta_{1}$

$\delta^{*}$

$\Delta \eta, \Delta \xi$

$\epsilon$

$\bar{\epsilon}$

$\tilde{\epsilon}$

$\theta$

$\tilde{\theta}$

$\lambda$

$\mu$

$\mu_{t}$

$\rho$

$\xi, \eta$

$\bar{\xi}$

$\omega$

Subscripts

$i, j$

$w$

$\xi, \eta$

$\infty$

Superscripts

$+,-$

Upper, lower finite-difference cell

Dimensional, or lagged (guessed) quantity

Derivative with respect to independent variable

Triple-deck parameter, Eqs. (1) and (9)

Boundary-layer displacement thickness, Eq. (29)

Small parameter, Eq. (2)

Effective viscosity

Offset distance, Eq. (40)

Boundary-layer momentum thickness, Eq. (B.4c)

Weighting factor, Eq. (43)

Scaled upstream skin friction, Eq. (1)

Viscosity coefficient

Effective total viscosity coefficient, Eq. (B.1)

Density

Endpoint of inviscid integration subinterval

FLARE coefficient, Eq. (38)

Evaluated at location $i, j$

Evaluated at wall or in wake

Partial derivative in given coordinate direction

Evaluated at freestream conditions

Pressure gradient parameter for flow past baseline curve, Eq. (24)

Pressure gradient parameter for flow past displacement body, Eq. (24)

Normal, tangential distance between boundary-layer mesh points

Transformed boundary-layer (G örtler) tangential, normal coordinates, Eqs. (18) and (19) 


\section{INTRODUCTION}

The problem considered in the present study is the way in which the structure of the flow past a symmetric airfoil evolves from one which is fully attached when the airfoil is very thin to one for which the flow is massively separated, as the airfoil thickness increases. An understanding of the phenomenon of massive separation is important, since its onset generally corresponds to the limit of the effective range of operation of an airfoil. Beyond a simple reduction in efficiency, massive separation (stall) can lead to conditions which compromise the safe operation of an aircraft. One example is the phenomenon of tip stall, where a wing at incidence to the freestream flow experiences locally stalled flow near one of the wingtips. The associated loss of lift generates a large rolling moment on the vehicle which can induce a hazardous spin. In turbomachinery applications, stall which occurs on blades within an engine can lead a reduction in the efficiency of the engine and, in the worst case, to a loss of power, which again has the potential for creating a dangerous condition. In addition, with the recent interest in highly maneuverable aircraft, which operate at or near stalled conditions, an understanding of the massive separation phenomenon becomes increasingly important for the efficient design of such vehicles.

The present effort focuses on the fundamental phenomenon of steady, massive separation for symmetric non-lifting airfoils by analyzing the process by which the crossover from locally separated to massively separated flow occurs as the airfoil thickness is increased. The understanding gained through consideration of the symmetric problem will be useful in helping to understand massive separation induced by increasing the angle of attack of an airfoil through stall. Restricting the analysis to steady separation eliminates the need to predict the complex way in which the flow develops into the massive separation structure thereby allowing the present focus to be placed on the final steady state flow structure. Finally, the present study serves to lay the foundation for the future development of methods of analysis which can be applied to lifting airfoils.

The analysis which is employed herein is developed by assuming that boundary-layer theory applies in many cases up to and beyond the initial onset of massive separation; this assumption is supported by a number of theoretical studies which will be discussed later in this section. As a consequence of the boundarylayer assumption, relatively simple models have been developed for the analysis of massive separation on airfoils. The knowledge gained from these simple models can be utilized to assist in the development of algorithms relying upon the Navier-Stokes equations to represent stall phenomenon.

Two theoretical approaches are employed in this study to calculate massively separated flow over airfoils. In the first approach, the problem is viewed in the asymptotic limit of infinite Reynolds number by utilizing triple-deck theory, in conjunction with the generalized Cheng-Rott (Ref. 1) thin-airfoil theory for mixed boundary-value problems. The Sychev-Smith (Ref. 2) massive separation model, which assumes a Kirchhoff eddy coupled to a triple-deck structure at the separation point, is applied. The second approach allows for finite Reynolds number effects by utilizing interacting boundary-layer theory, wherein instead of explicitly inserting the triple-deck structure at separation as done in the asymptotic approach, the boundary-layer equations are solved allowing for strong interaction between the boundary layer and the entire inviscid flow. The same inviscid model applied in the triple-deck analysis is utilized to calculate finite Reynolds number massively separated flow, but within the framework of an interacting boundary-layer numerical approach. Finite Reynolds number small scale local separation is also calculated in the interacting boundary-layer analysis, using the usual form of the incompressible thin-airfoil integral equation to represent the inviscid flow.

Triple-deck and interacting boundary-layer calculations are performed for two selected airfoil geometries for which the airfoil thickness is systematically varied in order to determine the way in which the flow becomes massively separated after initially experiencing small-scale local separation. The interacting boundary-layer procedure utilized in the present study to analyze the phenomenon of the finite Reynolds number crossover between local and massive separation is based upon that developed by Rothmayer and Davis (Ref. 3).

The methods of analysis developed herein are shown to be very useful for predicting the crossover behavior occurring for realistic airfoils. In particular, the crossover behavior predicted by both the triple-deck and interacting boundary-layer analyses are in qualitative agreement for the realistic airfoils considered here. In addition, the present interacting boundary-layer analysis has been applied to the crossover problem in flows with turbulence and in cases where boundary-layer control (suction and injection) is applied. Interacting 
boundary-layer theory is shown to be an efficient tool for the analysis of the crossover between local and massive separation on airfoils.

\section{Theoretical Basis for a Model of Stall}

Since boundary-layer theory provides the foundation for this investigation, important insights into the theoretical basis for the model of stall which is applied herein can be obtained by considering the evolutionary development of those aspects of the theory which are relevant to the present study. As shall be shown, the present approach is a natural extension of the evolving capability of boundary-layer theory.

The development of boundary-layer theory from its inception about eighty years ago has followed a slow but methodical path leading to a growing understanding of the phenomenon of boundary-layer separation. In comparison with the first six decades of the history of boundary-layer theory, the last twenty years have seen a quantum leap in the level of understanding of the physics associated with laminar flow separation, buit theie is still the need for considerably more innovative work before the phenomenon of boundary-layer separation can be considered to be well in hand. In the discussion which follows, which is summarized in Table 1, the fundamental developments in boundary-layer theory which have had the greatest impact on the still evolving understanding of boundary-layer separation are discussed. In each row of Table 1 the principal developments are illustrated. The first column shows the class of problems which is considered at each stage of the chronology, and lists the principal contributors in each area and the time frame of the initial developments in those areas. In the second and third columns, the framework in which boundary-layer theory has been applied in the asymptotic (infinite Reynolds number) limit and at finite Reynolds number, respectively, is noted at each stage in the chronology.

\section{Attached Flow}

The chronology begins with the first row of Table 1, where the genesis of boundary-layer theory is illustrated. Boundary-layer theory has its origins at the beginning of this century, with Prandtl's initial contribution in 1904 (Ref. 4).

Starting with the complete Navier-Stokes equations, Prandtl showed through an order-of-magnitude analysis that, in the limit of infinite Reynolds number, the first-order equations which result are the inviscid (Euler) form of the Navier-Stokes equations. This set of equations had been known in Prandtl's time to result in discrepancies between theoretical predictions and the experimentally observed behavior of real flows, which could be traced to the neglect of viscous effects. The presence of very small viscosity can cause the behavior of the flow to be greatly altered from that of a purely inviscid flow, and this is due to the formation of a "boundary layer" in the neighborhood of a solid boundary. The boundary layer forms because the fluid adheres to a solid surface (in a continuum sense), which is referred to as the "no-slip" condition.

The mathematical nature of the Euler equations does not permit the satisfaction of the no-slip condition at a solid surface. In order to satisfy the no-slip condition, boundary-layer theory postulates that a thin boundary layer develops between the outer inviscid flow and the body surface within which diffusion is important, and through which the inviscid surface velocity is reduced to zero at the body surface. Mathematically, the boundary-layer equations are developed from an order-of-magnitude analysis of the Navier-Stokes equations by retaining the next higher-order set of equations beyond the Euler equations, and taking the infinite Reynolds number limit. Upon matching the flow variables at the interface between the outer inviscid flow and the boundary layer, which in the limit of infinite Reynolds number is at the body surface, Prandtl's classical boundary-layer theory results. As the theory is described above, it is strictly an asymptotic theory applicable in the limit of infinite Reynolds number.

An important and restrictive feature of classical boundary-layer theory is the assumption that a hierarchy exists between the viscous and inviscid regions of the flow wherein the effect of the viscous flow upon the inviscid flow is higher order. The hierarchical nature of the flow is utilized to develop the finite Reynolds number application of classical boundary-layer theory, which is sometimes referred to as "weak interaction theory". In a typical application using the weak interaction approach, the inviscid surface pressure distribution is utilized to calculate the boundary-layer flow properties, in particular the displacement thickness. The displacement thickness represents the amount by which the original body has been "thickened" by the 
presence of the boundary layer, and is used to generate the body which is effectively "seen" by the inviscid flow, which consists of the original body plus the displacement thickness, that is, the "displacement body". The inviscid flow over the new effective body (and its viscous wake) is then recomputed yielding a new pressure distribution. If desired, this newly obtained pressure distribution can be utilized to recompute the boundary-layer properties and update the displacement body. This procedure can be repeated in an iterative fashion until a converged solution is (hopefully) obtained. One central point to note is that it is generally possible to extend classical boundary-layer theory, and its application to finite Reynolds number flow, to turbulent flow with the inclusion of a model for the turbulence.

TABLE 1

BOUNDARY-LAYER THEORY CHRONOLOGY

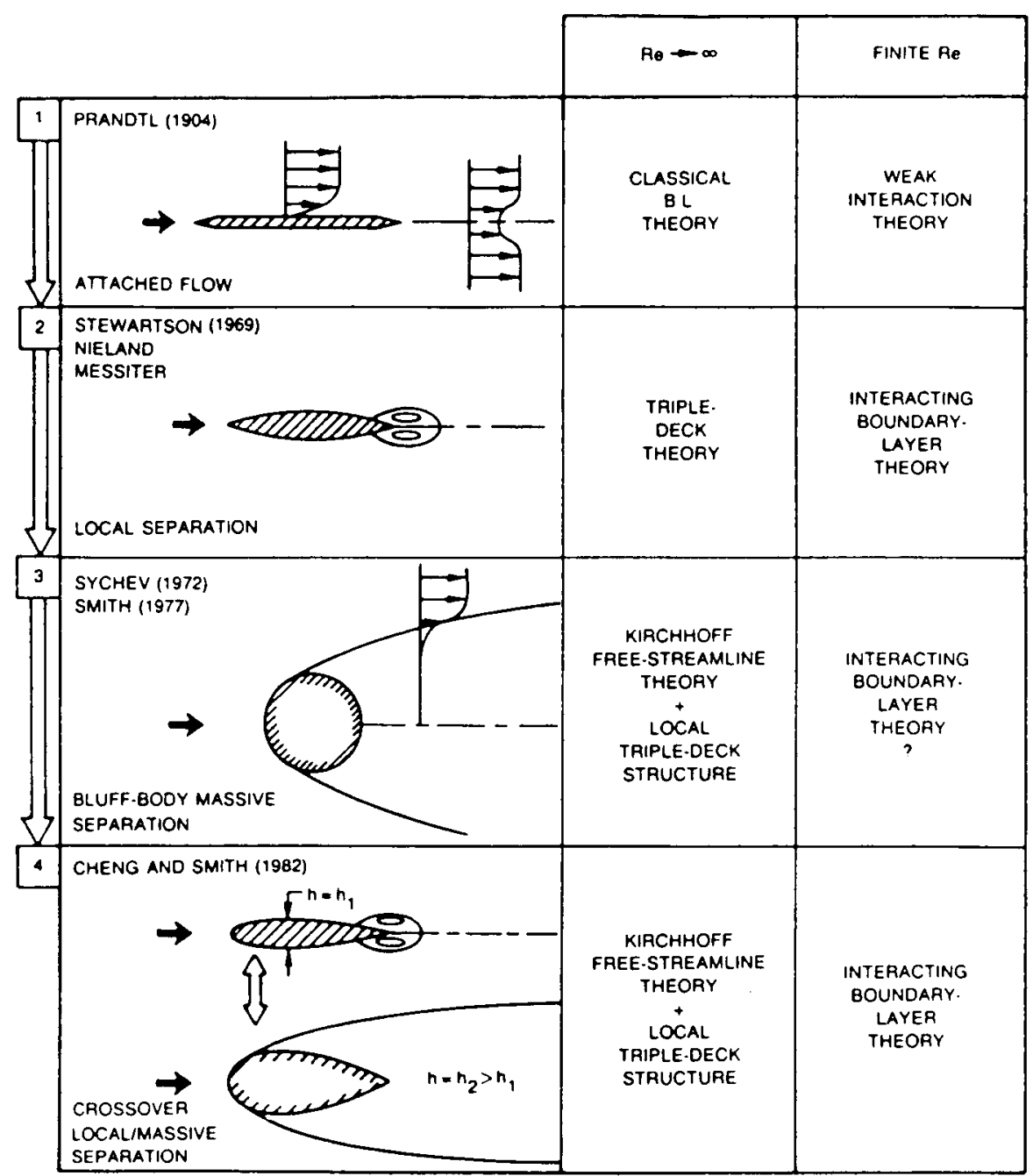

Local Separation

As is well known, in a large number of cases the weak interaction procedure described above ultimately terminates when the boundary layer encounters what is known as the Goldstein singularity when an apparent separation point is approached. The fundamental reason for the emergence of the Goldstein singularity is the lack of a mechanism whereby the effect of the viscous flow upon the inviscid flow can be raised to first order, as usually occurs, for example, when a boundary layer separates from a solid surface. 
A number of years elapsed between the development of Prandtl's classical boundary-layer theory and the developments of the late 1960's which led to a theoretical understanding of the mechanism which permits the "strong" interaction between the viscous boundary layer and the outer inviscid flow which is required to circumvent the appearance of the Goldstein singularity (second row of Table 1). This strong interaction is characteristic of flow in the vicinity of a separation point and heralds the breakdown of the hierarchy assumed previously between the viscous and inviscid flow regions in the weak interaction theory. The formal theory resulting from the work on strong viscous-inviscid interaction has become known as "triple-deck theory", in reference to the three tiered structure which arises in the boundary-layer and nearby inviscid flow when the interaction between the viscous flow and the inviscid flow becomes strong. The principal developers of this theory in the late 1960's were Neiland, Stewartson and Messiter (Refs. 5-7), although others played an important role.

Triple-deck theory owes much to the work of Lighthill (Ref. 8) in the 1950's, who studied the mechanism whereby information can propagate upstream through the boundary layer, in an effort to explain experimental data which showed that that the boundary layer can respond upstream of the location of an imposed disturbance, even when the inviscid flow is entirely supersonic. The existance of upstream propagation of information in a supersonic flow is in direct contradiction to the mathematical nature of the equations which govern such flows, since the supersonic inviscid flow is governed by hyperbolic equations and the boundary layer is governed by parabolic equations.

Triple-deck theory is an asymptotic theory derived, as Prandtl derived classical boundary-layer theory, from the Navier-Stokes equations in the asymptotic limit of infinite Reynolds number. The theory results from the application of the appropriate matching conditions for the asymptotic behavior of the flow variables upstream and downstream as well as matching in the direction normal to the body surface. Triple-deck theory removes the Goldstein singularity by permitting the viscous and inviscid flows to simultaneously adjust, allowing a smooth passage of the solution through the point of boundary-layer separation.

Even before triple-deck theory had been developed, analytical techniques were developed and applied to finite Reynolds number flow problems in which strong interactions exist between the viscous boundary layer and the outer inviscid flow. The development of triple-deck theory gave those procedures, collectively known as "interacting boundary-layer theory", a firm theoretical basis in the asymptotic setting of infinite Reynolds number. Interacting boundary-layer theory can be viewed as the reasonable finite Reynolds number analog to the rational infinite Reynolds number asymptotic theory, triple-deck theory.

As with weak interaction theory, extensions to turbulent flow of interacting boundary-layer theory have generally been found to yield reasonable results (within the limitation of the turbulence model utilized), even if the scalings which apply are no longer those of the laminar triple deck.

\section{Bluff-Body Massive Separation}

The next reference point in the present boundary-layer chronology, represented in the third row of Table 1 , is the work on bluff-body massive separation which followed closely the development of triple-deck theory. In 1972 Sychev published a paper in which he proposed a structure to explain the mechanism whereby a laminar boundary layer can break away from a smooth surface resulting in massive separation (Ref. 9). A century earlier Kirchhoff (Ref. 10) had contributed free streamline theory, an inviscid theory, in an effort to model the phenomenon of bluff body separation. In this model the free streamlines are assumed to be boundaries across which there is a discontinuity in the velocity, from freestream velocity on the external side to zero velocity on the internal side; the free streamlines bound the so-called "dead-air region" behind the bluff body within which the fluid is assumed to be very nearly static with respect to the body.

Free streamline theory alone is not sufficient to close the problem of massive separation, for it is not a consistent model as it does not properly account for the role of viscous effects near the separation site. Sychev's contribution was the proposal of a mechanism which, by coupling Kirchhoff's free streamline theory with the assumption that a triple-deck structure arises locally in the vicinity of the separation point, allows for a consistent model for both the viscous and inviscid flows, at least on the body scale (i.e., on a length scale comparable to the airfoil chord), for massive separation. Thus Sychev formulated a mathematical model for the phenomenon of massive separation.

Smith, in a landmark paper in 1977 (Ref. 2), addressed the critical questions of existence and uniqueness 
of a solution to the mathematical problem which Sychev posed (Ref. 9). By using a numerical approach, Smith showed fairly conclusively that a solution exists to Sychev's problem and that it is unique. Smith's numerical results were further verified by the later calculations of Korolev (Ref. 11) and Van Dommelen and Shen (Ref. 12). Smith worked out details of the embedding of triple-deck theory into the Kirchhoff free streamline model in a paper published in 1979 (Ref. 13). Thus a possible structure describing the phenomenon of massive separation from bluff bodies had been shown to exist. Inherent in that description is the assumption that the internal structure of the eddy downstream of separation is rather benign, that is, it has no significant impact on the flow behavior on the scale of the body $(\mathrm{x}=O(1))$.

In the Sychev-Smith bluff-body massive separation model, the separated flow region is represented as a constant pressure region extending, in the limit of infinite Reynolds number, infinitely far downstream of the separation point. The major deficiency in this model is the lack of a mechanism to account for closure of the eddy (Ref. 14). Since publication of his 1977 paper (Ref. 2), Smith has considered the structure of the eddy, including closure, and he has found that the structure may not be as benign as assumed in the original theory incorporating the Kirchhoff eddy model. However, the correct behavior of the flow on the body scale is believed to be captured by the Sychev-Smith model.

Although Kirchhoff free-streamline theory has been considered in the work discussed above, another classical inviscid model for the eddy structure is the Prandtl-Batchelor model, wherein the massively separated flow region behind the body is postulated to consist principally of a pair of inviscid constant vorticity eddies. This model thus far lacks any inviscid solutions for smooth bodies, and there is currently no experimental or numerical evidence in its favor. However, it is notable that the latest proposed structure of a massively separated laminar flow given by Smith (Ref. 14) incorporates the Prandtl-Batchelor structure in the downstream portion of the eddy, which Smith postulates is a region of nearly constant vorticity, while near the body the eddy boundary grows in a parabolic (Kirchhoff-like) manner. Thus elements of both the Kirchhoff eddy (on the body scale) and the Prandtl-Batchelor eddy (on the larger eddy scale) are present in the structure of a massively separated flow recently proposed by Smith (Ref. 14). The structure which is postulated in Ref. 14 is the first one which has been proposed for which it appears possible, in an asymptotic sense, to match all of the various regions of the eddy, from breakaway on the body surface to eddy closure far downstream. However, it remains to be proven that a massive separation solution exists which has all of the properties of that proposed structure. One encouraging sign is found in the low Reynolds number Navier-Stokes results obtained by Fornberg (Ref. 15) for flow past a circular cylinder, which show a tendency toward some of the features of the massively separated flow structure postulated by Smith (Ref. 14). Fornberg's results are discussed further below.

An important observation to be made regarding the Sychev-Smith bluff-body massive separation theory is that the triple-deck structure arising at separation is a local structure and as such it is essentially independent of the geometry of the body being considered. The location of the separation point is a function of the body geometry, however. The triple-deck structure in the vicinity of the separation point will retain its local character independent of the form of the downstream eddy; therefore whatever model is used for the eddy, essentially the same triple-deck structure will be embedded within it.

With regard to the determination of a structure for massive separation which is consistent from the separation point through closure of the eddy, the model for internal flows has a firmer basis. Smith (Ref. 16) has shown that the Kirchhoff model of the eddy gives a consistent account of massive separation in a cascade. In such flows the closure of the eddy occurs through a viscous-inviscid interaction, as opposed to the principally inviscid mechanism acting in an external flow. Thus Smith has determined the first fully consistent high Reynolds number structure for a massively separated flow, namely that occurring in a cascade of airfoils.

The problem of bluff-body massive separation at high (but finite) Reynolds number is at present far from being well in hand for external flows. In fact for the classic case of laminar flow past a circular cylinder, reliable numerical calculations recently obtained by Fornberg (Ref. 15) with symmetry imposed to force a steady state solution of the Navier-Stokes equations, are only available up to a Reynolds number of 600 , far from the high Reynolds number regime. However, even at a Reynolds number of 600 , eddy closure occurs at about 85 cylinder radii downstream of the body. In the structure of the eddy proposed recently by Smith (Ref. 14), the eddy dimensions are $O(\mathrm{Re})$ length by $O(\mathrm{Re})$ width with nonuniform vorticity immediately behind the body generated by a reentrant jet returning upstream along the wake centerline and emanating 
from the closure at the rear of the massive eddy. The calculation of such a massive and complicated structure at high Reynolds number clearly poses a monumental task, since the flow on the scale of the massive eddy would have to be computed at the same time as resolving the very small spatial scales associated with the triple-deck structure in the immediate vicinity of separation.

As will be shown later in this study, it is possible to address problems in high Reynolds number bluffbody massive separation using interacting boundary-layer theory, however there are some aspects of such an approach which need to be addressed before an interacting boundary-layer model for this phenomenon can be considered to be reasonable. Because of the questionable ability of interacting boundary-layer theory to handle the bluff-body massive separation problem at present, the finite Reynolds number entry in the third row of Table 1 is accompanied by a question mark.

\section{Crossover Between Local and Massive Separation}

The last area of the boundary-layer chronoiogyy, represented in the fourth row of Table 1 , is recent work which addresses the problem which is considered in the present study, namely the crossover process between local laminar separation on thin bodies and laminar massive separation occurring for flow past bluff bodies. In the paper by Cheng and Smith (Ref. 17), the crossover problem is considered for flow at infinite Reynolds number. The problem was analyzed in terms of a scaled thickness parameter which is given by the physical thickness multiplied by a function of the Reynolds number. Increasing (or decreasing) this parameter is therefore equivalent to increasing (or decreasing) either the body thickness or the Reynolds number. The fundamental assumption which is made in Ref. 17 is that the Sychev-Smith model for bluff-body massive separation applies essentially unaltered to thin bodies, once massive separation has occurred. That is, a local triple-deck structure, the precise form of which was determined by Smith (Ref. 2), arises in the vicinity of the separation point, and the flow downstream of separation is viewed as consisting of a constant pressure Kirchhoff eddy with parabolic growth far downstream. Using this model, the evolution of the separation structure as it changes from the local form occurring when the airfoil thickness parameter is small to the massive separation structure present when the thickness parameter is sufficiently increased is studied in Ref. 17.

An important finding of the triple-deck analysis which was performed in Ref. 17 is that the crossover between local and massive separation occurs when the airfoil thickness is $O\left(R e^{-1 / 16}\right)$, at which point the disturbance to the inviscid flow due to the local triple-deck structure at separation is of the same order of magnitude as the disturbance induced by the airfoil itself. Cheng and Smith (Ref. 17) examined the way in which the Kirchhoff eddy, of large $\left(O\left(R e^{3 / 4}\right)\right)$ length, collapses to an eddy of $O(1)$ length as the airfoil thickness parameter decreases, or conversely, how the eddy grows from a local bubble to the massively separated form as the thickness parameter increases. Airfoils with different trailing-edge geometries, either cusped or wedge-shaped, are considered in Ref. 17, and significant qualitative differences in the crossover behavior are predicted by the asymptotic theory. In particular, some very interesting phenomena can arise when the airfoil has a cusped trailing edge.

The qualitative behavior described in Ref. 17 for the two different trailing-edge geometries is discussed below, and is illustrated in Figs. 1 and 2, which are adapted from Ref. 17. In Figs. 1 and 2, the abscissa represents the location of the separation and reattachment (or wake closure) points, $x_{s}$ and $x_{r}$, respectively, measured from the airfoil leading edge, and the ordinate represents the scaled thickness parameter, $\widetilde{h}$, which is a measure of the airfoil thickness at fixed Reynolds number, or the Reynolds number at fixed airfoil thickness.

First consider an airfoil which has a wedge-shaped trailing edge for which the crossover process is illustrated in Fig. 1. For a wedge-shaped trailing-edge airfoil, increasing the thickness parameter causes the flow to initially separate at some non-zero value of the parameter, equal to $\widetilde{h}_{s}$. Continuing to increase $\widetilde{h}$ causes the separation bubble to grow from a small $\left(O\left(R e^{-3 / 8}\right)\right)$ length bubble at the trailing edge to an $O(1)$ length until a critical value of the thickness parameter, $\widetilde{h}_{c}$, dependent on the airfoil geometry, is reached. If the thickness parameter is increased slightly beyond this critical value, the eddy "inflates" becoming much longer and thicker, eventually $O(\mathrm{Re})$ by $O(\mathrm{Re})$, according to Smith (Ref. 14).

If instead of increasing the airfoil thickness parameter from a value $\tilde{h}_{\tilde{h}}<\tilde{h}_{c}$ for which a small scale local separation bubble exists, the airfoil thickness decreases from a value $\tilde{h}>\widetilde{h}_{c}$ for which the flow is 
massively separated, the eddy deflates following in reverse exactly the same path as when the eddy inflates as the thickness parameter increases through $\widehat{h}_{c}$. The initial point at which the parabolically growing free streamlines collapse to the symmetry line at downstream infinity as the airfoil thickness parameter decreases has been referred to as "cut-off" by Cheng and Smith (Ref. 17). The cut-off behavior for a wedge-shaped trailing edge occurs in a continuous and steady state fashion, and is unique. That is, given a value of the airfoil thickness parameter, a unique location is predicted for the separation and reattachment points.

In contrast to the behavior described above for a wedge-shaped trailing edge, a cusped trailing-edge airfoil displays significantly more complicated behavior than the wedge trailing-edge geometry as the thickness parameter is varied, with the crossover from local to massive separation occurring in a discontinuous fashion, leading to phenomena such as hysteresis and non-uniqueness which are not observed for wedgeshaped trailing-edge airfoils. The typical sort of behavior predicted for cusped trailing-edge airfoils by the asymptotic theory is illustrated in Fig. 2.

In an effort to describe the behavior which is possible for cusped trailing-edge airfoils, the separation and reattachment point locations are presented in Fig. 2 as a function of the airfoil thickness parameter. The airfoil thickness parameter is initially assumed to have the value $\widetilde{h}_{1}$, for which the flow is massively separated. If $\widetilde{h}$ is gradually decreased from $\widetilde{h}_{1}$, the massive eddy slowly shrinks until the points denoted "2" on the figure are reached. A decrease in the thickness parameter slightly below the value at " 2 " forces the separation and reattachment points to jump discontinuously to the points labeled " 3 ", where the flow is no longer massively separated, the eddy having collapsed to a localized separated region. A further reduction in $\vec{h}$ causes the bubble to collapse to a point on the airfoil surface upstream of the trailing edge when $h$ has the value $\widetilde{h}_{s}$. If, instead of decreasing the thickness parameter from its value at " 3 ", $\widetilde{h}$ is increased, the local bubble will grow in extent until the points labeled " 4 " are reached. An increase in $\tilde{h}$ slightly beyond the value at "4" again forces a discontinuous jump, this time from a locally to a massively separated flow, to the points labeled "5". Thus a path for hysteresis can be set up going around the loop 2-3-4-5-2 in Fig. 2. In addition, the possibility exists that for a cusped trailing-edge airfoil, attached flow can be maintained for an airfoil thickness up to $O(1)$. The possible existence of nonuniqueness is indicated in Fig. 2 for values of $\widetilde{h}$ corresponding to the region between the points labeled 2 and 5 , where both a local and a massive separation solution can exist, as well as by the possibility of an attached flow solution which may exist for the entire range of airfoil thicknesses up to $O(1)$. Cheng and Smith (Ref. 17) also point out that the crossover from the local to the massive separation structure, that is, going from points 2 to 3 or from 4 to 5 , probably occurs in an unsteady fashion for cusped trailing-edge airfoils.

Cheng (Refs. 18 and 19) has considered infinite Reynolds number laminar separation from asymmetric airfoils and airfoils at angle of attack. Further intriguing phenomena such as symmetry breaking, that is, the existence of asymmetric massive separation for a symmetric airfoil at zero angle of attack, have been predicted. In addition, Rothmayer and Smith (Ref. 20) have considered the crossover from massive separation to an attached flow in a cascade of airfoils using the same basic asymptotic approach described above, but adapted to the cascade problem. The principal contributions of that study are the demonstration of the importance of local geometry effects in determining the location of the separation point and the properties associated with the cut-off phenomenon, as well as the possible occurrence of multiple regions of hysteresis in cascades. The effect of cascade spacing has been shown in Ref. 20 to have a strong influence on massive separation phenomena.

The finite Reynolds number application of the asymptotic analysis of Cheng and Smith (Ref. 17) has been carried out by Rothmayer and Davis (Ref. 3), using interacting boundary-layer theory in conjunction with the assumption that the massive eddy is of the Kirchhoff (constant pressure) form, to study the crossover process on model airfoil geometries. The only change to the "standard" interacting boundary-layer theory approach for incompressible flow over thin airfoils is to replace the usual Cauchy integral representing the inviscid flow with the Cheng-Rott integral for mixed boundary-value problems (Ref. 1). An important feature of this approach to the massive separation problem is that the Cheng-Rott integral need only span a finite region extending from the airfoil leading edge to a location just downstream of the separation point, as opposed to the semi-infinite region over which the Cauchy integral must be applied. Rothmayer and Davis (Ref. 3) have observed many of the same phenomena at finite Reynolds number which were predicted by Cheng and Smith using the asymptotic theory (Ref. 17), including non-uniqueness and an indication of the unsteady crossover between the local and massive separation structures for cusped trailing-edge airfoils. 
The evolution of boundary-layer theory is seen above to have followed a logical progression which has allowed the consideration of successively more complex phenomena associated with boundary-layer separation. In particular, the analysis of the complicated means by which the crossover between local and massive separation occurs has recently become possible. The principal objective of the present effort is to extend the procedure developed by Rothmayer and Davis (Ref. 3) for the analysis of the crossover process to permit the investigation of crossover for realistic airfoil geometries, and to include the effects of turbulence and boundary-layer control. 


\section{PROBLEM DESCRIPTION AND FORMULATION}

The phenomenon of crossover between local separation and massive separation which occurs as the thickness of an airfoil is increased is investigated in the present study for laminar incompressible flows by utilizing two different approaches. The first approach employed is to consider the problem in the asymptotic limit of infinite Reynolds number. The theory which applies in this limit is known as Triple-Deck Theory. The second approach utilized allows consideration of the problem at finite (but large) values of the Reynolds number. The theory which is applied for the finite Reynolds number analysis is known as Interacting Boundary-Layer Theory. By employing these two theories in concert, one can hopefully obtain a more thorough understanding of the crossover phenomenon, as the knowledge of the limiting behavior of a physical phenomenon often aids in understanding the behavior when the limiting parameter, in this case the Reynolds number, is far from its asymptotic value. The present analyses are developed for application to symmetric airfoils operating at zero angle of attack.

\section{Triple-Deck Theory}

One of the goals of the present study is to determine the behavior of the separated flow structure as a function of airfoil thickness for realistic airfoils. In order to accomplish this goal, the asymptotic behavior of the separation point location has been determined by utilizing triple-deck theory, following the analysis presented by Cheng and Smith (Ref. 17). The results of the triple-deck analysis will provide guidance in interpreting the finite Reynolds number behavior which will be determined later using interacting boundarylayer theory.

Two fundamental assumptions are made in the asymptotic analysis with regard to the structure of a massively separated flow. The first is that the eddy which arises downstream of the boundary-layer breakaway point contains a weak recirculating current, and therefore the pressure is assumed to be a constant throughout the eddy. This model for the eddy is consistent with Kirchhoff's (Ref. 10) free streamline theory, which assumes that the eddy is open. The second assumption follows that adopted by Sychev (Ref. 9), whereby separation is assumed to occur within a triple-deck structure. The reasoning which lead to the introduction of the latter assumption will be described below.

Let us consider the impact upon the results of classical boundary-layer theory of varying the assumed location of the breakaway point, which will be denoted by $x_{0}$ in this discussion. The pressure gradient imposed upon the boundary layer is assumed to be that which results from the inviscid (free streamline) theory (e.g., see Ref. 21). In the discussion which follows, the body is assumed to be symmetric and bluff, e.g., a circular cylinder, and $x$ is measured along the body surface from the leading edge stagnation point.

The behavior of the free streamline downstream of the separation point is discussed first, in order to determine the limitations on the possible location of the separation point due to purely inviscid considerations. The furthest possible upstream location of the separation point, based upon free streamline theory, is the location designated $x_{s}$; if the separation point is upstream of $x_{s}$ the free streamlines will cut through the body surface, which is clearly not physically plausible. In addition, if the separation point is assumed to be downstream of the point designated here as $x_{c}\left(x_{c}>x_{s}\right)$, then the symetrically disposed free streamlines will intersect one another at a finite distance downstream of the body, which also is not physically plausible. The conclusion which is reached from purely inviscid considerations is that the separation point must be located between the two points $x_{s}$ and $x_{c}$.

Purely inviscid considerations determined from free streamline theory impose upstream and downstream limits on the location of the separation point. The pressure gradient which the inviscid flow imposes upon the boundary layer, in conjunction with classical boundary-layer theory, will hopefully be sufficient to determine the precise location of the separation point. The pressure gradient immediately upstream of the separation point is of the form $d p / d x \sim-k\left(x_{o}-x\right)^{-1 / 2}$, where $k$ is a nonpositive constant which is a function of the assumed location of separation. The furthest possible upstream location of the separation point determined from inviscid considerations is at $x_{o}=x_{s}$; this point is known as the smooth separation point. The smooth separation point corresponds to the location at which $k$ is equal to zero, that is, the pressure gradient is zero at $x_{s}$. Since boundary-layer separation must occur in an adverse pressure gradient, the separation point cannot be at $x_{s}$. If the separation point is assumed to occur aft of $x_{s}$, where $k<0$, an infinitely 
adverse pressure gradient will exist at $x_{o}$. The pressure gradient just upstream of $x_{o}$ is extremely adverse, and therefore classical boundary-layer theory will predict that separation will occur ahead of the assumed separation point at $x_{o}$. Thus, viscous considerations do not permit separation to occur in the region in which the separation point must be located based upon inviscid considerations.

A paradoxical situation is seen to arise when free streamline theory for the inviscid flow is coupled to classical boundary-layer theory, since any point on the body surface at which separation is assumed to occur leads to an incompatibility between the inviscid and the viscous theories. In order to resolve this apparent paradox, Sychev (Ref. 9) proposed that a triple-deck structure at the separation point smooths out the infinitely adverse pressure gradient predicted by the inviscid theory for $x_{0}>x_{s}$, and permits the boundary layer to pass smoothly through the separation point by allowing for a locally strong interaction between the inviscid and the viscous flows. Within Sychev's proposed structure, the pressure upstream of the separation point behaves according to Eq. (1), which contains a dependence upon the Reynolds number through the definition of $\epsilon$, Eq. (2). The dependence is such that as the Reynolds number approaches the asymptotic limit, $x_{0} \rightarrow x_{s}$. Thus the proposed structure provides a means to overcome the paradox described above, and provides a mechanism by which Reynolds number dependence is introduced into the massive separation problem thereby affecting the location of the separation point.

The laminar separation criterion on the pressure immediately upstream of the separation point was proposed by Sychev (Ref. 9) to be of the form

$$
p-p_{e} \sim-\alpha \lambda^{9 / 8} \epsilon^{1 / 2}\left(x_{0}-x\right)^{1 / 2}
$$

where $x$ is the arc length measured along the surface from the stagnation point and is normalized by a characteristic length scale, typically the airfoil chord, $x_{o}$ is the separation point location, $p$ is the pressure, normalized by twice the freestream dynamic pressure $\rho U_{\infty}^{2}, p_{e}$ is the (constant) pressure in the eddy downstream of separation, $\lambda$ is the wall shear at $x=x_{0}$ which is evaluated using classical (weak-interaction) boundary-layer theory, and $\alpha$ is the positive constant determined by Smith (Ref. 2) to have the value 0.44 . The parameter $\epsilon$ is given in terms of the Reynolds number,

$$
\epsilon=R e^{-1 / 8}=\left(\frac{\rho U_{\infty} L}{\mu}\right)^{-1 / 8}
$$

and becomes vanishingly small in the asymptotic limit, as $R e \rightarrow \infty$. The separation criterion (Eq. (1)), although determined for bluff body massive separation, applies to thinner bodies as well. Therefore Eq. (1) forms the basis for the asymptotic analysis of massive separation on realistic symmetric airfoils which follows.

The triple-deck analysis is based upon the assumption that the massively separated flow structure on the body scale $(x=O(1))$ can be modeled for slender bodies by utilizing thin-airfoil theory. The coordinate system for the triple-deck analysis has its origin at the airfoil leading edge with the $x$-axis oriented along the chord line, and $x$ increasing in the direction of the trailing edge, and the $y$-axis is normal to the oncoming uniform flow, positive in the upward direction. The coordinates $(x, y)$ are nondimensionalized by the airfoil chord. The separation point is located at the point $x_{o}$ where $0<x_{o}<1$. Upstream of the separation point the body shape is specified, and it is represented by normal injection through the $x$-axis. The body shape is written in the form

$$
y=R e^{-1 / 16} \tilde{h} f(x)
$$

where the function $f(x)$ represents the body profile, and $\tilde{h}$ is the airfoil thickness parameter which is $O(1)$, so that the physical airfoil thickness is $O\left(R e^{-1 / 16}\right)$; thus the airfoil is thin when the Reynolds number is large.

Downstream of $x_{0}$ the assumption is made that the eddy is of the Kirchhoff form and the pressure is a constant, equal to the freestream value, within the eddy and along the free streamline. The assumption of constant pressure along with thin-airfoil theory implies that downstream of the separation point a constant value of the tangential velocity is specified along the $x$-axis. In addition, it is assumed that the free streamline shape is given by 


$$
y=R e^{-1 / 16} S(x)
$$

where the quantity $S(x)$ is $O(1)$ so that the free streamline is slender when the Reynolds number is large. The resultant thin-airfoil problem is of a mixed boundary-value type, for which the form of the solution has been determined by Cheng and Rott (Ref. 1).

The crucial element needed to complete the statement of the problem is the inclusion of the triple-deck separation criterion, Eq. (1), which is applied at the point $x_{o}$, with $p_{e}$ equal to $p_{\infty}$. Utilizing complex-variable theory leads to the following mathematical statement of the problem,

$$
\widetilde{p}(x, 0)+i S^{\prime}(x)=\frac{i}{\pi}\left(x-x_{o}\right)^{1 / 2} \int_{0}^{x_{o}} \frac{\widetilde{h} f^{\prime}(s)}{\left(x_{o}-s\right)^{1 / 2}(x-s)} d s
$$

where $\tilde{p}$ is the $O(1)$ scaled pressure related to the nondimensional physical pressure by

$$
p=R e^{-1 / 16} \tilde{p}
$$

and' denotes differentiation with respect to the independent variable. Solving for the real part of this expression for $x<x_{o}$ yields the following expression for the pressure,

$$
\widetilde{p}(x, 0)=-\frac{\widetilde{h}}{\pi}\left(x_{o}-x\right)^{1 / 2} \int_{0}^{x_{o}} \frac{f^{\prime}(s)}{\left(x_{o}-s\right)^{1 / 2}(x-s)} d s, \quad x<x_{o}
$$

and solving for the imaginary part for $x>x_{0}$ results in the derivative of the free streamline shape,

$$
S^{\prime}(x)=\frac{\tilde{h}}{\pi}\left(x-x_{0}\right)^{1 / 2} \int_{0}^{x_{0}} \frac{f^{\prime}(s)}{\left(x_{0}-s\right)^{1 / 2}(x-s)} d s, \quad x>x_{0}
$$

By applying the triple-deck criterion for the behavior of the pressure at the separation point given by Eq. (1) to Eq. (7) the following condition for determining the position of separation is obtained

$$
\int_{0}^{x_{o}} \frac{f^{\prime}(s)}{\left(x_{o}-s\right)^{3 / 2}} d s=\frac{\alpha \lambda^{9 / 8} \pi}{x_{o}^{9 / 16} \tilde{h}}
$$

From Eq. (8) it can be seen that as $x \rightarrow \infty$

$$
S(x) \sim b x^{1 / 2}, b=\frac{2 \tilde{h}}{\pi} \int_{0}^{x_{\circ}} \frac{f^{\prime}(s)}{\left(x_{o}-s\right)^{1 / 2}} d s
$$

that is, far downstream the eddy grows parabolically. There is generally a value of $x_{0}<1$ for which $b$ is equal to zero; this value, designated $x_{o_{c}}$, corresponds to the phenomenon which Cheng and Smith refer to as "cut-off", which they discuss in detail in Ref. 17, and which was described briefly in the Introduction above. For values of $x_{o}>x_{o_{c}}, b<0$, and for $x_{o}<x_{o_{c}}, b>0$. The massive separation model given by $\mathrm{Eq}$. (9) only applies for $b>0$. When $b<0$ the massive separation structure no longer exists, the flow having crossed over to an entirely different flow structure with the eddy dimensions significantly diminished from those applying when the flow is massively separated.

The behavior of the separation point as a function of the airfoil thickness parameter, $\widetilde{h}$, is sought. It is easier in practice to solve Eq. (9) as an inverse problem by specifying $x_{0}$ and obtaining the corresponding value of $\widetilde{h}$. One reason for the use of an inverse procedure is the possiblity of nonuniqueness in the functional dependence of $x_{0}$ on $\widetilde{h}$, which is easily determined by solving in an inverse fashion, since the dependence of $\widetilde{h}$ upon $x_{0}$ is unique. The reader is referred to the detailed discussion of the nonuniqueness/bifurcation phenomenon which is provided in Ref. 17; the present discussion will be limited to a brief summary of some of the relevant conclusions of that study.

In the study of Cheng and Smith (Ref. 17), certain airfoil profiles were found to give rise to nonuniqueness/bifurcation in the behavior of the separation point as a function of the airfoil thickness parameter. In particular, airfoils with cusped trailing edges, as opposed to wedge-shaped trailing edges, were found to yield bifurcation. Rothmayer (Ref. 22) discusses the possibility that a more general criterion for nonuniqueness 
than the presence of a cusped trailing edge exists, and demonstrates that the crossover properties (between local and massive separation) tends to be governed by the local curvature of the airfoil surface in the vicinity of the separation point, rather than simply by whether the trailing edge is cusped or wedge-shaped. Based upon the discussion and the results which were presented in Ref. 22 , it appears that an inflection point in the airfoil profile near the trailing edge is sufficient to induce nonuniqueness and/or hysteresis in many airfoils; this issue will be interrogated further in the present study.

The asymptotic analysis is carried out in the present investigation by assuming that the airfoil profile function $f(x)$ is specified to be of the particular form

$$
f(x)=a_{0} x^{1 / 2}+a_{1} x+a_{2} x^{2}+a_{3} x^{3}+a_{4} x^{4}
$$

where the coefficients $a_{0}$ through $a_{4}$ are determined for each airfoil under consideration. The expression given by Eq. (11) corresponds to the exact analytical form of the NACA 4 and 5 digit series airfoil thickness distributions, and has been found herein to give a good representation of other airfoil thickness distributions when utilized in a least-squares curve fit procedure using discrete data given for the airfoil profile. Assuming $f(x)$ to be written in the form of Eq. (11) allows Eqs. (9) and (10) to be solved analytically. The solutions to these equations are given in Appendix $\mathrm{A}$. It is notable that since the analytical function given by $\mathrm{Eq}$. (11) provides a good fit to many general airfoil thickness distributions, and leads to an exact analytical integration of Eq. (9), the result provided in Appendix A is very useful for solving the asymptotic symmetric massive separation problem in a very straightforward manner for many symmetric airfoil geometries, once they are fit to the profile function given by Eq. (11).

\section{Interacting Boundary-Layer Theory}

Central to the interacting boundary-layer approach is the assumption that when the flow Reynolds number is sufficiently high, the flowfield can be subdivided into two regions, an outer inviscid region and an inner viscous region. These two regions are coupled through the mutual interaction existing between them, which is induced by the displacement thickness effect of the viscous flow upon the inviscid flow, and the simultaneous modification of the viscous flow by changes in the inviscid flow. In interacting boundary-layer theory, the effect of the viscous and inviscid regions of the flow upon one another is assumed to be of equal order of magnitude, in contrast with classical boundary-layer theory, where the effect of the viscous flow upon the inviscid flow is assumed to be weak, that is, of higher order.

The simultaneous influence of one region of the flow upon the other which is present in the strong interaction problem must be reflected in the numerical solution procedure which is developed to solve for flows which contain regions of strong viscous-inviscid interaction. The basic approach usually involves replacement of the specification of the pressure with specification of the displacement thickness or the skin friction in the boundary-layer calculation, i.e., an "inverse" boundary-layer calculation procedure is employed. The use of an inverse boundary-layer procedure eliminates the possible occurrence of the Goldstein singularity, which would terminate "direct" boundary-layer calculations, i.e., calculations in which the pressure is specified.

Another issue which must be addressed is the method used to couple the inviscid solution technique to the viscous solution procedure in a manner which honors the non-hierarchical nature of a strong viscousinviscid interaction. Several different procedures which accomplish this goal have been developed and some representative examples of successful coupling techniques are given in Refs. 23-26.

\section{Viscous Flow Model}

The viscous flow at high Reynolds number is confined to thin shear layers which are present on the surface of a body, along the boundary of a separated flow region and in the wake behind a finite body. These shear layers are in general dominated by diffusion in a single direction; diffusion is negligible normal to that direction. The equations which govern this type of viscous flow are the boundary-layer equations developed by Prandtl (Ref. 4). In the present study, the incompressible boundary-layer equations are written in a coordinate system which is located a small distance from the body surface or wake centerline (but within the shear layer). This "shear-layer" or "baseline" coordinate system was introduced by Werle and Verdon (Ref. 27 ), and its principal use is to permit better alignment of the boundary-layer coordinate system with the 
shear layer than is obtained with a coordinate system which is body-oriented. Proper choice of the shearlayer coordinates leads to honoring the boundary-layer approximations more accurately, since neglecting diffusion along a properly chosen base curve should be more accurate than doing so along the tangent to the body surface, particularly when the flow is separated. The introduction of a baseline coordinate system leads to one difficulty however, and that is that the body surface does not lie along a constant coordinate curve. Fortunately, this difficulty is easily overcome by applying the Prandtl transposition theorem.

In the following discussion, an asterisk denotes a dimensional quantity. The independent and dependent variables appearing in the boundary-layer equations are nondimensionalized and the Prandtl transposition theorem is applied simultaneously to yield

$$
s=s^{*} / L^{*}, n=\left(y^{*}-f^{*}\right) \sqrt{R e} / L^{*}, u=u^{*} / U_{\infty}^{*}, v=\left(w^{*}-\frac{d f^{*}}{d x^{*}} u^{*}\right) \frac{\sqrt{R e}}{U_{\infty}^{*}}
$$

and

$$
p=\frac{p^{*}-p_{\infty}^{*}}{\rho^{*} U_{\infty}^{* 2}}
$$

where $s^{*}$ is the distance along the baseline coordinate curve measured from the leading edge, $x^{*}$ is the distance along the horizontal axis which is aligned with the oncoming uniform flow and has its origin at the leading edge, $n^{*}$ is measured from the body surface, $y^{*}$ is measured from the base surface (both along a line normal to the base surface) and $f^{*}$ is the distance from the base curve to the body surface along the normal to the base curve (positive in the direction of the outward normal); the nondimensionalized $f$ is scaled by the square root of the Reynolds number in the same way that $n$ is defined. In addition, $L^{*}$ is the reference length (taken to be the airfoil chord in the present study), the subscript $\infty$ indicates the freestream value and the Reynolds number, Re, is defined by

$$
R e=\frac{\rho^{*} U_{\infty}^{*} L^{*}}{\mu^{*}}
$$

The transposition theorem appears in the definition of the normal velocity, $v$, and the normal coordinate, $n$. The quantity $w^{*}$ is the physical velocity normal to the $x$-axis. Refer to Fig. 3 for an illustration of the coordinate definitions and the shear-layer coordinate.

Utilizing the above variables, the boundary-layer equations for the conservation of mass and momentum assume the identical form as when they are written in body oriented coordinates. Since the equations can be found in many standard works (e.g., Schlichting (Ref. 28)), they will not be repeated here.

In addition to the governing equations, boundary conditions must be prescribed. On a solid surface no slip is usually assumed and normal injection is allowed,

$$
u(x, 0)=0
$$

and

$$
v(x, 0)=v_{w}(x)
$$

where $v_{w}(x)$ is the specified distribution of the surface injection velocity. If a nonzero slip velocity is introduced, the right-hand side of Eq. (14a) is set to the appropriate value of $u$. On a symmetric wake centerline symmetry is imposed on the $u$-component of velocity,

$$
\frac{\partial u}{\partial z}(x, 0)=0
$$

where $z$ is oriented normal to the $x$-axis, and the wake centerline is a streamline so that the normal velocity is zero,

$$
w(x, 0)=0
$$

At the outer edge of the boundary layer the tangential velocity approaches the inviscid velocity at the displacement body surface, 


$$
u(x, n) \rightarrow U_{e}(x) \text { as } n \rightarrow \infty
$$

From the matching condition at the outer edge (see Van Dyke (Ref. 29)) it can be shown that, with surface injection,

$$
v-v_{w}-n v_{n} \rightarrow \frac{d}{d x}\left(U_{e} \delta^{*}\right) \text { as } n \rightarrow \infty
$$

where $\delta^{*}$ is the displacement thickness measured from the body surface. This condition is more convenient to use for the purpose of coupling the viscous and inviscid equations than that given by Eq. (15), as shall be seen later. In addition, the matching conditions provide the dimensionless inviscid surface normal injection velocity, $W(x, 0)$, which is applied along the base coordinate curve,

$$
W(x, \hat{0})=\frac{1}{\sqrt{R e}} \frac{d}{d x}\left[U_{e}\left(\delta^{*}+f\right)\right]
$$

In order to provide more efficient and potentially more accurate solutions to the boundary-layer equations, similarity type variables are introduced in place of the physical variables. Because similarity variables take advantage of the locally similar nature of the flow, their use minimizes streamwise gradients. In addition, similarity variables account for the growth of a laminar boundary layer thereby allowing the use of a fixed number of grid points in the normal direction throughout the entire flowfield. The similarity variables utilized in the present study are a modified form of the Görtler variables. Let $U_{e_{0}}$ be the inviscid surface speed along the baseline coordinate surface. The independent transformed variables are defined by

$$
\xi=\int_{0}^{s} U_{e_{0}}(t) d t
$$

and

$$
\eta=\frac{U_{e_{o}}(s)}{\sqrt{2 \xi}} n
$$

where $s$ is the arc length measured along the baseline curve from the leading edge. Defining transformed velocities by

$$
u=U_{e_{o}} F
$$

and

$$
v=\frac{U_{e_{o}}}{\sqrt{2 \xi}} V-\frac{\partial \eta}{\partial s} \sqrt{2 \xi} F
$$

where $V$ is a similarity velocity function, the continuity and momentum equations in transformed variables become

Continuity:

$$
V_{\eta}+F+2 \xi F_{\xi}=0
$$

and

Momentum:

$$
\left(\bar{\epsilon} F_{\eta}\right)_{\eta}-V F_{\eta}+\beta_{1}-\beta_{o} F^{2}-2 \xi F F_{\xi}=0
$$

where $\beta_{o}$ and $\beta_{1}$ are defined by

$$
\beta_{o}=\frac{2 \xi}{U_{e_{o}}} \frac{d U_{e_{o}}}{d \xi}
$$

and 


$$
\beta_{1}=\frac{2 \xi}{U_{e_{o}}^{2}} U_{e} \frac{d U_{e}}{d \xi}
$$

The quantity $\beta_{0}$ is the pressure gradient parameter for the inviscid flow over the baseline coordinate surface and $\beta_{1}$ is the pressure gradient parameter for the interacted flow; $U_{e}$ is the inviscid surface speed for flow past the "effective" body, that is, the body thickened by the viscous displacement thickness. The momentum equation has been written in a general form which allows for the introduction of turbulence through the term $\bar{\epsilon}=1+\epsilon$ where here $\epsilon$ is the turbulent eddy viscosity coefficient, which is zero for laminar flow, and is defined for turbulent flow in Appendix B.

The boundary conditions must also be transformed into similarity variables; the solid surface boundary conditions become

$$
F(\xi, 0)=0
$$

and

$$
V(\xi, 0)=V_{w}(\xi)
$$

where the transformed surface injection velocity is determined from the physical variable using Eq. (21) evaluated at the surface. As before, if a nonzero slip velocity is assumed at the surface, $F$ is set to the appropriate value there. The wake centerline boundary conditions transform to

$$
\frac{\partial F}{\partial \eta}(\xi, 0)=0
$$

and

$$
V(\xi, 0)=0
$$

The edge conditions equivalent to Eqs. (15) and (16) become

$$
F \rightarrow U_{e} / U_{e_{o}}
$$

and

$$
V-\eta V_{\eta} \rightarrow \sqrt{2 \xi} \frac{d}{d \xi}\left(U_{e} \delta^{*}\right)+V_{w}
$$

which are evaluated at the edge value of $\eta, \eta_{\max }$. The displacement thickness, $\delta^{*}$, is defined in transformed variables by

$$
\delta^{*}=\frac{\sqrt{2 \xi}}{U_{e_{o}}} \int_{0}^{\infty}\left(1-\frac{U_{e_{o}}}{U_{e}} F\right) d \eta
$$

\section{Inviscid Flow Model}

The inviscid flow is modeled in the present investigation by assuming that the flow can be represented using thin-airfoil theory. Two inviscid models are developed, the first is applicable to attached flows and flows with relatively small separation bubbles, that is, flows for which localized regions of strong interaction are present. This model will be referred to as the Local Strong-Interaction (LSI) model. The second model is applicable to configurations for which massive separation occurs and will be referred to as the Massive Separation (MS) model.

In the local strong-interaction model, classical thin-airfoil theory for incompressible flow is employed. In the following discussion it will assumed for clarity that the baseline coordinate surface is the $x$-axis, which is aligned with the oncoming freestream flow. This assumption will be relaxed subsequently. For the thin-airfoil analysis the assumption is made that boundary conditions at the airfoil surface can be transferred to the baseline coordinate surface. The condition for the inviscid surface injection velocity $W(x, 0)$ which is applied 
along the $x$-axis is given by Eq. (17). With this condition and the inviscid flowfield governed by thin-airfoil theory, $W(x, 0)$ is related to $U_{e}(x)$ by the Cauchy principal value integral (see Ashley and Landahl (Ref. $30)$ ),

$$
U_{e}(x)=1+\frac{1}{\pi} \int_{0}^{\infty} \frac{W(t, 0)}{x-t} d t
$$

which is written to reflect the fact that the displacement thickness is zero upstream of the leading edge at $x=0$.

In the massive separation model the inviscid flow is divided into two regions. The first is the region extending from the leading edge to a prescribed point on the airfoil surface at $x=x_{0}\left(0<x_{0}<1\right)$ over which the displacement body is prescribed. In the second region, where $x>x_{o}$, the pressure is assumed to be a constant, which corresponds to the Kirchhoff eddy. Effectively, the inviscid normal injection velocity is prescribed in the upstream region and the tangential velocity is prescribed in the downstream region. Cheng and Kott (Ref. 1) have developed a generalized method for mixed boundary-value problems in thinairfoil theory which is applicable to this problem. Rothmayer and Davis (Ref. 3) have shown how to apply this generalized method to the massive separation problem and they obtained the inviscid expression which applies in this case, relating the inviscid surface speed on the displacement body to the normal injection velocity distribution along the $x$-axis. The condition which applies is

$$
U_{e}(x)=1+\frac{\sqrt{x_{0}-x}}{\pi} \int_{0}^{x_{0}} \frac{W(t, 0)}{(x-t) \sqrt{x_{0}-t}} d t, x<x_{0}
$$

which reflects the fact that the displacement thickness is zero upstream of the leading edge. Note that as $x_{0} \rightarrow \infty$, Eq. (31) reduces to Eq. (30), which is the standard thin-airfoil equation for the edge velocity. Recalling that $W(x, 0)$ is given by Eq. (17), it has been shown (Ref. 3 ) that the method of Cheng and Rott (Ref. 1) leads to the following expression valid for $x>x_{o}$ :

$$
\frac{d}{d x}\left[U_{e}\left(\delta^{*}+f\right)\right]=\frac{\sqrt{x-x_{0}}}{\pi} \int_{0}^{x_{0}} \frac{\frac{d}{d t}\left[U_{e}\left(\delta^{*}+f\right)\right]}{(x-t) \sqrt{x_{o}-t}} d t
$$

One of the goals of the present study is to apply the interacting boundary-layer analysis to realistic airfoils. Because most realistic airfoils have blunt leading edges, thin-airfoil theory cannot be applied in its usual form, since the theory is not valid locally near the leading edge of a blunt-nosed airfoil. In order to remedy this shortfall of thin-airfoil theory, instead of assuming that the flow is a small perturbation to a uniform stream (i.e., flow past a flat plate), the assumption will be made that the flow over a blunt-nosed airfoil can be represented as a small perturbation to the flow past a parabola which has the same nose radius as the airfoil under consideration, which is accomplished by choosing the baseline coordinate curve to be the osculating parabola to the nose of the airfoil. The inviscid flow about a parabola can be solved analytically by using conformal mapping to map the parabola to a stagnation plane, which was the approach employed by Rothmayer (Ref. 22) to calculate the massively separated flow past elliptical cylinders using interacting boundary-layer theory. In the present study the parabola mapping is applied, in addition to the massive separation calculations, to the local strong-interaction calculations in order to permit the use of interacting boundary-layer theory in conjunction with thin-airfoil theory for calculations of symmetric flow past realistic airfoils for which the flow is attached or mildly separated. Rothmayer presents a procedure which can be applied to solve a more general class of airfoil problems within the context of the Cauchy integral (Ref. 22).

The details of the mapping are contained in Ref. 22, hence only the final results will be shown here. The osculating parabola to an airfoil of nose radius $r_{o}$ is defined by the equation

$$
y=\sqrt{2 r_{o} x}
$$

Mapping this parabola from the complex $z$-plane $(z=x+i y)$ to the $\bar{\xi}$-axis of the stagnation plane $(\zeta=\bar{\xi}+i \bar{\eta})$ results in the following expressions for the pertinent variables needed for the interacting boundary-layer equations:

$$
U_{e_{o}}(\xi)=\left[\frac{2 \xi}{2 \xi+r_{o}}\right]^{1 / 2}
$$


and

$$
\beta_{o}=\frac{r_{o}}{2 \xi+r_{o}}
$$

For a parabola, the value of the Görtler variable $\xi$ is equal to the value of the Cartesian $x$-coordinate at that location. For a given value of $\xi$ (equal to $x$ ), the value of $s$, the arc length measured along the parabola from the leading edge, is given by

$$
s=\sqrt{\xi\left(\xi+\frac{r_{o}}{2}\right)}+\frac{r_{o}}{2} \ln \left[\sqrt{\frac{2 \xi}{r_{o}}+1}+\sqrt{\frac{2 \xi}{r_{o}}}\right]
$$

Because the parabola has been unfolded in the $\zeta$-plane, in the case where massive separation occurs there are two free streamlines, extending to $\bar{\xi}= \pm \infty$. In order to account for this, the method of Cheng and Rott (Ref. 1) must be modified. Rothmayer presents the resulting massive separation inviscid integrals, which have the following form after transforming back to the $z$-plane (Ref. 22):

$$
U_{e}(\xi)=U_{e_{o}}(\xi)\left[1+\frac{\sqrt{\xi_{o}-\xi}}{\pi} \int_{0}^{\xi_{o}} \frac{W(\xi, 0)}{(\xi-t) \sqrt{\xi_{o}-t}} d t+I(\xi)\right], 0<\xi<\xi_{0}
$$

and

$$
\frac{d}{d \xi}\left[U_{e}\left(\delta^{*}+f\right)\right]=\frac{\sqrt{\xi-\xi_{0}}}{\pi} \int_{0}^{\xi_{0}} \frac{\frac{d}{d t}\left[U_{e}\left(\delta^{*}+f\right)\right]}{(\xi-t) \sqrt{\xi_{o}-t}} d t+I(\xi), \xi>\xi_{0}
$$

where $I(\xi)$ is defined by

$$
I(\xi)=\frac{1}{\pi}\left[U_{e_{0}}\left(1+\frac{r_{o}}{2 \xi}\right)^{1 / 2}-1\right]\left[\frac{\pi}{2}+\sin ^{-1}\left(\frac{2 \xi}{\xi_{o}}-1\right)\right] U_{e_{o}}(\xi)
$$

where $U_{e}$, is the imposed constant velocity (i.e., pressure) in the massive separation eddy. Note that if $U_{e}$, is equal to unity (the freestream value), $I(\xi)$ does not vanish. The term $I(\xi)$ arises because the pressure is not equal to the freestream value at $\xi=\xi_{o}$; the freestream pressure is only achieved asymptotically along the parabola as $\xi \rightarrow \infty$. Note that these equations were developed in Ref. 22 with the assumption that $r_{o} \ll \xi_{o}$ , so that strictly speaking these equations are limited to thin, blunt leading-edge airfoils.

A procedure similar to that described above has been implemented in the present analysis to permit the application of the Cauchy integral, Eq. (30), to blunt leading-edge airfoils. Proceeding as was done for the massive separation integral, the Cauchy integral is transformed to

$$
U_{e}(\xi)=U_{e_{o}}(\xi)\left[1+\frac{1}{\pi} \int_{0}^{\infty} \frac{\frac{d}{d t}\left[U_{e}\left(\delta^{*}+f\right)\right]}{\xi-t} d t\right]
$$

where once again the base flow is assumed to be the flow past the osculating parabola. 


\section{NUMERICAL METHOD}

Having presented the equations which govern the viscous and inviscid flows, the numerical procedures which are employed to solve the equations will be discussed next. The method of solution for the viscous equations, wherein a finite-difference approach is utilized, is considered first. The viscous solution procedure for both the local strong-interaction and the massive separation problems is identical. The inviscid numerical methods are developed from the appropriate governing equation, Eq. (30) for the local strong-interaction problem, and Eq. (31) for the massive separation problem. An important consideration in the numerical solution of the interacting boundary-layer equations is the method by which the viscous and the inviscid flow equations are coupled. The coupling technique is discussed after the numerical approaches for each of the two regions are presented below.

\section{Viscous Equations}

The partial differential equations governing the viscous flow, (22) and (23), and the boundary conditions, (26) and (28), are solved using a finite-difference approach. The momentum equation (22) is recast by substituting from the continuity equation (23) to eliminate the $\xi$-derivative in the former equation. With the momentum equation in modified form, only the continuity equation contains a derivative with respect to $\xi$. Therefore, if the $\eta$-derivatives are all discretized to second-order accuracy, a completely second-order accurate scheme can be achieved by only considering the continuity equation.

The form of the momentum equation which results after substitution from the continuity equation is the following:

$$
\left(\bar{\epsilon} F_{\eta}\right)_{\eta}-V F_{\eta}+\omega F V_{\eta}+\beta_{1}+\left(\omega-\beta_{o}\right) F^{2}=0
$$

The factor $\omega$ has been introduced to allow for the application of the FLARE approximation (Ref. 31) to stabilize the equations in regions of reversed flow. If the use of FLARE is desired, then $\omega$ is set equal to zero wherever $F$ is negative, otherwise $\omega$ is equal to unity. It has been found, however, that because the $\xi$-derivative does not appear explicitly in the momentum equation, the equations can often be marched into the separated flow region, even if the flow is massively separated, with $\omega$ set equal to unity. In the present local strong-interaction calculations, FLARE has been found to ultimately be necessary to stabilize the solution when the separated region becomes very large, while in the massive separation calculations, where the solution is generally terminated a short distance aft of the separation point, the use of FLARE is usually unnecessary. Upwind differencing of the $\xi$-derivatives in the momentum equation has been attempted in the local strong-interaction procedure by reverting to the momentum equation in its form prior to substitution from the continuity equation. For mildly separated flows, both FLARE and upwinding were found to give results which were very similar, however when the separation becomes very severe, with bubble lengths on the order of the airfoil chord, upwinding has been found to not be sufficient to stabilize the numerical scheme, while the FLARE approximation permits such cases to be converged. The difficulties encountered in converging separated solutions which employ upwinding were unexpected, and should be interrogated further in the future.

In order to discretize the boundary-layer equations, the finite-difference mesh must first be defined. In Fig. 4, a typical computational molecule is illustrated with $i$ and $j$ denoting the indices in the $\xi$ - and $\eta$ directions, respectively. For the purpose of developing a general scheme, the grid spacing in the $\eta$-direction is assumed to vary. The scheme developed herein will be first-order accurate in $\Delta \xi$ and second-order accurate in $\Delta \eta$. If turbulence is included, the scheme will become first-order accurate in $\Delta \eta$ unless $\Delta \eta$ is constant or varying sufficiently slowly, or unless the eddy viscosity coefficient is a constant in the $\eta$-direction.

The continuity equation, (22), is discretized first, at the point $j+1 / 2$, which yields an expression of the form

$$
V_{i, j-1}=V_{i, j}+P_{j}\left(F_{i, j}+F_{i, j-1}\right)-Q_{j}
$$

The coefficients $P_{j}$ and $Q_{j}$ are defined in Appendix C.

Discretization of the momentum equation in the form (38) is considered next. The momentum equation must be considered carefully in order to obtain second-order accuracy on a grid with arbitrarily varying 
$\Delta \eta$. The assumption is made that only three grid points in the $\eta$-direction are to be employed in the finitedifference molecule. The most restrictive condition for second-order accuracy can be shown to arise from the first term in Eq. (38), the second derivative. An analysis to determine a second-order accurate representation for this term leads to the conclusion that, for an arbitarily varying $\Delta \eta$ grid, the second derivative must be evaluated at an "offset" point which does not coincide with the grid point $j$. The offset point is located a distance $\tilde{\epsilon}$ away from $j$, where $\tilde{\epsilon}$ is positive when the offset is in the direction of $j+1$. The value of the quantity $\tilde{\epsilon}$ is determined from the expression

$$
\tilde{\epsilon}=\frac{\Delta \eta^{+}-\Delta \eta^{-}}{3}
$$

where $\Delta \eta^{+}=\left(\eta_{j+1}-\eta_{j}\right)$ and $\Delta \eta^{-}$is defined in a like manner by decrementing $j$ by one in the expression for $\Delta \eta^{+}$. The resulting expression for the second derivative is

$$
\left(\bar{\epsilon} F_{\eta}\right)_{\eta}=\frac{2}{\Delta \eta^{+}+\Delta \eta^{-}}\left[\frac{\bar{\epsilon}_{j+1 / 2}}{\Delta \eta^{+}}\left(F_{i, j+1}-F_{i, j}\right)-\frac{\bar{\epsilon}_{j-1 / 2}}{\Delta \eta^{-}}\left(F_{i, j}-F_{i, j-1}\right)\right]
$$

Second-order accuracy is achievable on a variable $\Delta \eta$ grid if $\bar{\epsilon}$ is a constant, i.e., either the flow is laminar or the eddy-viscosity coefficient is constant, or if the rate of variation of $\Delta \eta$ is small (formally, if $\Delta \eta^{+} / \Delta \eta^{-}-1=$ $O(\Delta \eta)$ ). For an arbitrarily varying grid spacing, it is not possible to obtain a second-order accurate expression for the second derivative with a variable coefficient $\bar{\epsilon}$ involving only three grid points. Most of the calculations performed herein have been for laminar flow, and the turbulent calculations have been carried out on grids which satisfy the small rate-of-variation criterion noted above, hence all of the results which are presented here are second-order accurate in $\Delta \eta$.

All remaining terms in the momentum equation, which consist of first derivative and function evaluations, must be evaluated at the offset point to maintain second-order accuracy in $\Delta \eta$, which is accomplished by writing all terms at the midpoint of each of the two cells, i.e., at $j \pm 1 / 2$ and then adding the expressions obtained for each cell in an appropriately weighted manner. The result for each of the terms at $j+1 / 2$ is as follows,

$$
\begin{gathered}
\left.V F_{\eta}\right|_{i, j+1 / 2}=\frac{1}{2 \Delta \eta^{+}}\left(V_{i, j+1}+V_{i, j}\right)\left(F_{i, j+1}-F_{i, j}\right) \\
\left.F V_{\eta}\right|_{i, j+1 / 2}=\frac{1}{2 \Delta \eta^{+}}\left(F_{i, j+1}+F_{i, j}\right)\left(V_{i, j+1}-V_{i, j}\right) \\
F_{i, j+1 / 2}^{2}=\frac{1}{2}\left(F_{i, j+1}^{2}+F_{i, j}^{2}\right)
\end{gathered}
$$

Similar forms are obtained at $j-1 / 2$ by decrementing $j$ by one in Eqs. (41b-d). After evaluating all of the terms in the momentum equation at $j \pm 1 / 2$, the upper and lower cell expressions are added in order to obtain the second-order accurate evaluation at the offset point. The form which results for a typical term evaluated at the offset point, for example, $V F_{\eta}$, is

$$
V F_{\eta}=\left.\tilde{\theta} V F_{\eta}\right|_{i, j+1 / 2}+\left.(1-\tilde{\theta}) V F_{\eta}\right|_{i, j-1 / 2}
$$

where the weighting function, $\tilde{\theta}$, is given by

$$
\tilde{\theta}=\frac{\Delta \eta^{-}+2 \widetilde{\epsilon}}{\Delta \eta^{-}+\Delta \eta^{+}}
$$

All of the terms are written at the offset point by using the weighting function.

Finally, the nonlinear terms in the momentum equation must be linearized, which is accomplished by assuming that any nonlinear term, which can be represented in the general form $U V$, can be linearized as

$$
U V=U^{*} V+U V^{*}-U^{*} V^{*}
$$


where the asterisk denotes a "guessed" value of the quantity, which will come either from the previous station $(i-1)$ or from the previous iteration. Since the interacting boundary-layer method requires global iteration, in the present procedure the guessed value of a quantity at $(i, j)$ is always that from the previous global iteration at the same $(i, j)$ location, except during the first global sweep, when the guessed value is that from the previous station, i.e., $(i-1, j)$.

After discretization and linearization, all of the common terms in Eq. (38) are collected to yield an equation of the form

$$
\bar{A}_{j} F_{i, j-1}+\bar{B}_{j} F_{i, j}+\bar{C}_{j} F_{i, j+1}+\bar{D}_{j} V_{i, j-1}+\bar{E}_{j} V_{i, j}+\bar{F}_{j} V_{i, j+1}+\bar{G}_{j} \beta_{1 i}=\bar{H}_{j}
$$

where the coefficients are defined in Appendix C.

The boundary conditions must also be written in discrete form. At a solid surface, where the index $j$ is equal to one, the boundary conditions, given by Eq. (26), are written as

$$
F_{i, 1}=0
$$

and

$$
V_{i, 1}=V_{w}\left(\xi_{i}\right)
$$

At a symmetric wake centerline, where $V=0$, symmetry and antisymmetry conditions on $F$ and $V$, respectively, are employed in place of Eq. (26c) (forcing satisfaction of (26c)) within the momentum equation written at the centerline, $j=1$. With the index of the point below the centerline denoted by $j=-2$,

$$
F_{i,-2}=F_{i, 2} \quad \text { and } \quad V_{i,-2}=-V_{i, 2}
$$

resulting in the following form for the momentum equation after linearization,

$$
A_{o} F_{i, 1}+B_{o} F_{i, 2}+C_{o} V_{i, 2}+D_{o} \beta_{1,}=E_{o}
$$

where the coefficients are defined in Appendix D. At the outer boundary, where $j$ is equal to $N J$, the boundary condition given by Eq. (28), is discretized to obtain

$$
\begin{aligned}
& V_{i, N J}-\eta_{N J}\left(V_{i, N J}-V_{i, N J-1}\right) /\left(\eta_{N J}-\eta_{N J-1}\right) \\
= & \sqrt{2 \xi_{i}}\left[\left(U_{e} \delta^{*}\right)_{i}-\left(U_{e} \delta^{*}\right)_{i-1}\right] /\left(\xi_{i}-\xi_{i-1}\right)+V_{w}\left(\xi_{i}\right)
\end{aligned}
$$

Note that the term $V_{\eta}$ is evaluated exactly since $V$ behaves linearly at the edge of the boundary layer. The definition of $F, \mathrm{Eq}$. (20), will also be employed at the edge of the boundary layer, where

$$
F_{i, N J}=U_{e_{i}} / U_{e_{o_{i}}}
$$

The momentum equation evaluated at the edge of the boundary layer is utilized to close the system of equations. Since $F_{\eta}$ and $\left(\bar{\epsilon} F_{\eta}\right)_{\eta}$ go to zero at the edge of the boundary layer, using Eq. (49) the momentum equation evaluated there can be shown to be equivalent to the definition of $\beta_{1}$. Thus the finite-difference form of Eq. (38), evaluated at the edge of the boundary layer, is used to provide the final relation needed for closure, and it is written in discrete form, after linearization, as

$$
\bar{W} F_{i, N J}+\bar{X} V_{i, N J}+\bar{Y} V_{i, N J-1}+\beta_{1_{i}}=\bar{S}
$$

where the coefficients are defined in Appendix D.

The set of finite-difference relations given by Eqs. (39), (45), and (46)-(50) provide a balance between the number of equations and the number of unknowns only if the displacement thickness at $i$ is known. In the present numerical approach, however, $\delta_{i}^{*}$ is unknown, and the final relation needed to close the system of finite difference equations will be obtained from the appropriate inviscid relation, either Eq. (30) or Eq. $(31)$. 
The system of equations which results from the procedure outlined above can be written in the form of a tridiagonal matrix, the solution for which can be efficiently obtained using recursion relations. The solution procedure will be described following the discretization of the inviscid governing equations, which is outlined below.

\section{Inviscid Equations}

The equations governing the inviscid flow for each of the solution techniques (local strong-interaction and massive separation) must be discretized to permit their application within the interacting boundary-layer approach. The appropriate discrete inviscid equation is coupled to the boundary-layer equations to yield an efficient system of finite-difference equations which can be solved in a relatively straightforward manner.

\section{Local Strong-Interaction Procedure}

Discretization of the Cauchy integral, which is used in the local strong-interaction solution procedure, is first considered. The discretization is carried out for the form given by Eq. (30); the discrete form of Eq. (37) is virtually identical, except that in the latter case the discretization is performed in terms of the Görtler variable and, on the left-hand side, $U_{e}$ is replaced by $U_{e} / U_{e_{o}}$.

The solution domain for Eq. (30) is generally divided into three regions; the first region extends from $x=0$ to $x=x_{l}$, the second spans the region between $x_{t}$ and $x_{F}$, and the final region extends from $x_{F}$ to $x=\infty$. The strong interaction is assumed to be confined to the region $x \subset\left(x_{I}, x_{F}\right)$; the other two regions are assumed to be weak-interaction regions for which the behavior of the displacement thickness is either available in a known analytic form or obtained from a separate numerical solution. In the present investigation, since all of the bodies considered have blunt leading edges, the strong-interaction region will begin at the leading-edge stagnation point, i.e., $x_{I}=0$. Therefore, only the downstream weak-interaction region is included in the present analysis.

The variable $T$ is introduced and defined by

$$
T(x)=R e^{-1 / 2} U_{e}\left\{\delta^{*}(x)+f(x)\right\}
$$

so that Eq. (30) can be written at $x=x_{i}$ as

$$
U_{e}\left(x_{i}\right)=1+\frac{1}{\pi} \int_{0}^{\infty} \frac{T^{\prime}(t)}{x_{i}-t} d t
$$

Rothmayer (Ref. 22) found that unless $T$ is rewritten by replacing $U_{e} f$ by $U_{e_{o}} f$, difficulties could arise in the numerics. There is no inconsistency introduced by the substitution, since it is consistent with the order of approximation introduced by the use of thin airfoil theory, hence $T$ is redefined as

$$
T(x)=R e^{-1 / 2}\left\{U_{e} \delta^{*}(x)+U_{e_{o}} f(x)\right\}
$$

to avoid the associated numerical difficulty.

For now, consider the integral spanning $\left(x_{I}, x_{F}\right)$. Integration by parts of Eq. (52), taking care with the treatment of the singular point at $x-t=0$, yields

$$
\int_{x_{I}}^{x_{F}} \frac{T^{\prime}(t)}{x_{i}-t} d t=\frac{T\left(x_{F}\right)}{x_{i}-x_{F}}-\frac{T\left(x_{I}\right)}{x_{i}-x_{I}}-\int_{x_{I}}^{x_{F}} T(t) d\left(\frac{1}{x_{i}-t}\right)
$$

This is similar to the integration by parts carried out by Davis and Werle (Ref. 26). The strong-interaction region is subdivided into subintervals, as shown in Fig. 5, within each of which $T$ is assumed to be a constant. The points at $x_{j}$ correspond to the boundary-layer solution points, and those at $\bar{x}_{j}$ are at the midpoints between the $x_{j}$, i.e., $\bar{x}_{j}=\left(x_{j}+x_{j-1}\right) / 2$. Each inviscid subinterval spans the region $x \subset\left(\bar{x}_{j-1}, \bar{x}_{j}\right)$. Note that the first boundary-layer solution point is at $x_{1}$ and the last is at $x_{I E}$, while the inviscid strong-interaction integral spans a slightly larger domain, starting and ending half a subinterval beyond the viscous calculation region. The last term in Eq. (54) is written as a summation over each individual subinterval with the assumption of constant $T$ within each interval to yield 


$$
\int_{x_{I}}^{x_{F}} T(t) d\left(\frac{1}{x_{i}-t}\right)=\sum_{j=1}^{I E} T_{j} D_{i j}
$$

where $T_{j} D_{i j}$ represents the integral over the $j$ th element

$$
D_{i j}=\frac{1}{x_{i}-\bar{x}_{j+1}}-\frac{1}{x_{i}-\bar{x}_{j}}
$$

The above form correctly accounts for evaluation of the Cauchy principal value of the integral (See Ref. 22).

Defining the qunatity $\bar{g}_{i}$ by the expression

$$
\bar{g}_{i}=1+\frac{1}{\pi}\left[\frac{T\left(x_{F}\right)}{x_{i}-x_{F}}-\frac{T\left(x_{I}\right)}{x_{i}-x_{I}}+\int_{0}^{x_{i}} \frac{T^{\prime}(t)}{x_{i}-t} d t+\int_{x_{F}}^{m} \frac{T^{\prime}(i)}{x_{i}-t} d t\right]
$$

Eq. (52) can be written (with $U_{e_{i}}=U_{e}\left(x_{i}\right)$ ) in the form

$$
U_{e_{i}}=\bar{g}_{i}+\frac{1}{\pi} \sum_{j=1}^{I E} T_{j} D_{i j}
$$

The form which $\bar{g}_{i}$ has for the different far wake displacement thickness distributions appropriate to laminar and turbulent wakes is given in Appendix E. In the present study, since $x_{I}=0$, the second and third terms on the right-hand side of (56) are zero; the second term is equal to zero since $T(0)=0$. This expression can be rewritten to isolate the contribution to the displacement thickness distribution at $i$ as

$$
U_{e_{i}}=\bar{g}_{i}+\frac{1}{\pi} \sum_{\substack{j=1 \\ j \neq i}}^{I E} T_{j} D_{i j}+\frac{1}{\pi \sqrt{R e}}\left[\left(U_{e} \delta^{*}\right)_{i}+\left(U_{e_{o}} f\right)_{i}\right] D_{i i}
$$

which can be written in compact form as

$$
U_{e_{i}}=\widetilde{C}_{i}+\widetilde{D}_{i}\left(U_{e} \delta^{*}\right)_{i}
$$

for which the coefficients $\widetilde{C}$ and $\widetilde{D}$ are easily obtained from Eq. (57b), and are given in Appendix E.

Equation $(57 \mathrm{c})$ is the necessary additional relation which was sought earlier in order to close the viscous finite-difference set of equations.

As indicated earlier, Eq. (57c) must be modified slightly for a blunt-nosed body to reflect the differences between Eqs. (30) and (37). As mentioned above, the modifications consist of replacing $U_{e}$ by $U_{e} / U_{e_{o}}$ on the left-hand side and substituting the streamwise Görtler variable $\xi$ for $x$.

In practice, for the general case in which the upstream and downstream weak-interaction regions are present, within which the displacement thickness has an assumed analytic behavior, the two stronginteraction region endpoint values of $T$ appearing in the first two terms of Eq. (54) are used to evaluate any unknown constants which appear in the analytic expressions for the displacement thickness distribution in the weak-interaction regions. In order to justify determining the displacement thickness distributions in the weak-interaction regions from the strong-interaction solution results at the endpoints, it is required that the boundaries of the strong-interaction region be placed sufficiently far upstream and downstream of the actual location of the localized strong viscous-inviscid interaction. The procedure described above absorbs the displacement thickness effects upon the strong-interaction region from the upstream and downstream weak-interaction regions into the first and last points of the latter region. The implementation of the above technique is manifested in the numerics through modification of the definitions of the $D_{i j}$ coefficients for $j=1,2$ and $j=I E-1, I E$. 


\section{Massive Separation Procedure}

The inviscid integral appropriate to the massive separation model employed in the present study, Eq. (31), is now considered. The procedure described below is applicable to sharp leading-edge geometries. In order to discretize the blunt leading-edge form of (31), namely (36a), the modifications are straightforward, hence only the discretization of Eq. (31) will be described in detail.

As before, the definition (53) is used to rewrite (31) in the form

$$
U_{e}\left(x_{i}\right)=1+\frac{1}{\pi} \int_{0}^{x_{0}} \frac{T^{\prime}(t)}{\left(x_{i}-t\right) \sqrt{x_{o}-t}} d t, x<x_{o}
$$

The integral has two singularities instead of the one present in Eq. (30); the first singularity is at $t=x_{i}$ and the second is at $t=x_{0}$. The first singularity is treated in a similar manner to that used in evaluating Eq. (30) to extract the Cauchy principal value, while the second requires special treatment. In the present investigation, the procedure developed by Rothmayer and Davis (Ref. 3) is followed very closely. They began by analytically extracting the singularity at $x_{o}$ by writing

$$
U_{e_{i}}=1+\frac{\sqrt{x_{o}-x_{i}}}{\pi} \int_{0}^{x_{o}} \frac{T^{\prime}(t)-P^{\prime}(t)}{\left(x_{i}-t\right) \sqrt{x_{o}-t}} d t+\frac{T^{\prime}\left(x_{o}\right)}{\pi} \ln \left[\frac{x_{o}^{1 / 2}-\left(x_{o}-x_{i}\right)^{1 / 2}}{x_{o}^{1 / 2}+\left(x_{o}-x_{i}\right)^{1 / 2}}\right], x<x_{o}
$$

where

$$
P(x)=T\left(x_{o}\right)+\left(x-x_{o}\right) T^{\prime}\left(x_{o}\right)
$$

Following Rothmayer and Davis (Ref. 3), the values of $T\left(x_{o}\right)$ and $T^{\prime}\left(x_{o}\right)$ are obtained by using second-order accurate extrapolations from the three solution stations ahead of $x_{0}$. In addition, Eq. (36b) can be integrated to give

$$
\left(U_{e} \delta^{*}\right)_{i}=R e^{1 / 2} T\left(x_{0}\right)-f_{i}+\frac{2 \sqrt{R e}}{\pi} \int_{0}^{x_{0}} \frac{T^{\prime}(t)}{\sqrt{x_{o}-t}}\left\{\sqrt{x_{0}-x_{i}}-\sqrt{x_{o}-t} \tan ^{-1}\left(\frac{x_{i}-x_{o}}{x_{o}-t}\right)^{1 / 2}\right\} d t, x_{i}>x_{0}
$$

Equation (59c) is not employed within the interacting boundary-layer calculation; it is only used after the solution is converged to determine the distribution of the displacement thickness downstream of $x_{0}$. The numerical solution of Eq. (59c) will be discussed in Appendix E.

The discretization of the Cheng-Rott integral is performed in much the same manner as was done for the Cauchy integral. The Cheng-Rott integral is discretized over a grid subdivided as in Fig. 5 and $U_{e_{i}}$ is again written in the form of Eq. (57c), after isolating the contribution to the inviscid integral from the displacement thickness at $i$. In the massive separation procedure, the "influence coefficients", $D_{i j}$ are given by

$$
D_{i j}=\frac{1}{\left(x_{i}-\bar{x}_{j-1}\right)\left(x_{o}-\bar{x}_{j-1}\right)^{1 / 2}}-\frac{1}{\left(x_{i}-\bar{x}_{j}\right)\left(x_{o}-\bar{x}_{j}\right)^{1 / 2}}
$$

where the integral (59a) is written as

$$
U_{e_{i}}=\bar{g}_{i}+\frac{\left(x_{o}-x_{i}\right)^{1 / 2}}{\pi} \sum_{j=1}^{M}\left(T_{j}-P_{j}\right) D_{i j}
$$

and $M$ is the index of the last boundary-layer solution station upstream of $x_{0}$. The term $\bar{g}_{i}$ and the coefficients $\widetilde{C}_{i}$ and $\widetilde{D}_{i}$ are given in Appendix E. 


\section{Interacting Boundary-Layer Equations}

The combined viscous and inviscid equations are referred to as the interacting boundary-layer equations. The inviscid equations must be coupled to the viscous equations in some fashion which will lead to solutions which converge upon global iteration of the complete system. Two numerical methods which have been successfully applied to couple the equations for local-strong interaction calculations are the so-called semiinverse method of Carter (Ref. 24) and the quasi-simultaneous methods of the Veldman-Davis (Refs. 25 and 26) type.

The semi-inverse method provides a simple technique with which to couple the viscous and inviscid flow regions. The quasi-simultaneous procedure, which was developed after the introduction of the semi-inverse method, has been shown to generally be the more preferable of the two approaches, because the quasisimultaneous procedure tends to converge more rapidly due to its more implicit nature, as well as being in general more robust; the latter is an especially important consideration when separated flows are being calculated. For the reasons cited above, the quasi-simultañeous approach is utilized in the present study.

The numerical implementation of the quasi-simultaneous coupling procedure which has been adopted for both the local strong-interaction and the massive separation models can be described in the same way. The two relations used in coupling the viscous and inviscid flows are given by Eqs. (48) for the boundary-layer equations and $(57 \mathrm{c})$ for the inviscid equations. Between these relations the quantity $\left(U_{e} \delta^{*}\right)_{i}$ is eliminated and Eq. (49) is then used to express $U_{e_{i}}$ in terms of $F_{i, N J}$. The complete set of algebraic relations for the discretized interacting boundary-layer equations is straightforwardly solved through the use of the appropriately chosen recursion relations.

The recursion relations are postulated to be of the form

$$
F_{i, j}=Z_{1 j}+P_{1 j} F_{i, j+1}+Q_{1 j} V_{i, j+1}+R_{1 j} \beta_{1 i}
$$

and

$$
V_{i, j}=Z_{2 j}+P_{2 j} F_{i, j+1}+Q_{2 j} V_{i, j+1}+R_{2 j} \beta_{1,}
$$

The computation of the recursion relations is started at the body surface or wake centerline at $j=1$ using the appropriate boundary conditions on $F$ and $V$. On a solid surface the no-slip condition on $F$ given by Eq. (46a) implies that all of the recursion relation coefficients in Eq. (62a) are zero there. If a slip condition is applied, then all of the coefficients are zero except for $Z_{1_{1}}$, which is set equal to the specified value of the slip velocity. The surface injection condition on $V$ implies that $Z_{2_{1}}=V_{w}(\xi)$ and the remaining coefficients are zero at the surface. On a wake centerline $V$ is equal to zero so that all of the coefficients in (62b) are zero. The coefficients of (62a) are immediately determined from Eq. (47b), since the latter can be manipulated into the form of Eq. (62a). The discrete forms of the continuity and momentum equations in the mesh interior can be manipulated through substitution to obtain the form of the recursion relation coefficients there; they are defined in terms of the coefficients of the governing equations in Appendix D.

Once the recursion relation coefficients have been determined by sweeping from the boundary at $j=1$, the edge and coupling relations, Eqs. (48), (49), (50) and (57c) are solved to determine $\beta_{1_{i}}$ and the remaining unknowns at the boundary-layer edge, including the displacement thickness and inviscid edge velocity. The details of the procedure at the edge of the boundary layer, which is where the coupling between the viscous and inviscid flows is accomplished, are provided in Appendix D. Once the unknowns at the edge of the boundary layer have been determined, the recursion relations are employed to solve for $F_{i, j}$ and $V_{i, j}$ by sweeping from the edge of the boundary layer at $j=N J$ to the lower boundary at $j=1$.

The viscous-inviscid interaction solution is determined by globally iterating upon the displacement thickness distribution starting with an initial guess for the distribution. Each spatial sweep of the boundarylayer equations is handled in a parabolic-like manner, marching the equations from an initial boundary-layer profile, which is generally a similarity profile. The maximum fractional change in the displacement thickness is monitored from one global iteration to the next until it falls below some preset tolerance, usually $10^{-5}$ for the present calculations, at which point the calculation is considered to be converged, and is terminated.

For all of the airfoils considered in the present investigation, stretched grids are employed in both the streamwise and normal directions. The streamwise stretching is employed to resolve both the leading-edge 
region of the blunt-nosed airfoils and, in the local strong-interaction calculations, the trailing-edge/nearwake region. The stretching uscd is a simple geometric one in which the stretching coefficients are precisely determined to give the desired number of grid points within a specified region with the stepsize at one end of the region specified. The stretching in the normal direction is also geometric, with the stepsize near the wall specified to allow the lower deck of the triple deck to be captured in the regions of strong viscousinviscid interaction in laminar calculations, and to resolve the laminar sublayer in turbulent boundary-layer calculations. 


\section{DISCUSSION OF RESULTS}

In the present study, results have been obtained to demonstrate the ability to predict, using interacting boundary-layer theory, the crossover process which occurs between a local airfoil trailing-edge separation bubble and the massive separation structure which develops as the thickness of a symmetric airfoil is increased in a laminar flow at finite Reynolds number. In addition to the interacting boundary-layer results, asymptotic (infinite Reynolds number) results have been obtained using a triple-deck analysis. The results of the two analyses are compared to determine the extent to which the triple-deck and interacting boundary-layer theory predictions are in qualitative agreement. The effects of turbulence and boundary-layer control on the crossover process have also been investigated.

Both the triple-deck and the interacting boundary-layer analyses have been applied to two realistic airfoil geometries, the first of which corresponds to the NACA four digit series airfoil, NACA 00XX, where $X X$ represents the maximum thickness of the airfoil expressed in percent of chord. The thickness distribution of the NACA $00 X X$ airfoil has been modified slightly from the original distribution given by Abbott and Von Doenhoff (Ref. 32) to obtain closure at the trailing edge. The second airfoil corresponds to the thickness distribution of the Garabedian and Korn (GK) airfoil with the designation 70-10-13 (Ref. 33). The upper and lower surface coordinates are given in Ref. 33 as a discrete distribution of points. For this study these points have been used to determine the airfoil thickness distribution (i.e., the airfoil without camber), which has then been fit in a least-squares sense to a polynomial of the form of Eq. (11). The function describing the GK 70-10-13 airfoil is multiplied by a scaling factor which is used to generate a family of airfoils designated GK 70-10-XX, where XX again represents the maximum percent thickness to chord ratio. In addition, the effects of turbulence and boundary-layer control have been investigated for the NACA 00XX airfoil series.

\section{Triple-Deck Analysis}

Before considering the results of the calculations performed using the finite Reynolds number (interacting boundary-layer) approach, the behavior predicted by triple-deck theory at the asymptotic condition of infinite Reynolds number is considered. Specifically, using the technique described in the previous section for the solution of Eq. (9), the behavior of the separation point has been predicted for thicknesses beyond the critical value at which cut-off (wake inflation) occurs. For the asymptotic analysis, the airfoil thickness is scaled by the Reynolds number to yield a thickness parameter, $\tilde{h}$, given by the expression

$$
\widetilde{h}=R e^{1 / 16} h
$$

The first result shown is that obtained for the NACA 00XX airfoil. The airfoil geometry is given by the expression

$$
f(x)=h\left(1.4845 \sqrt{x}-0.6405 x-1.758 x^{2}+1.4215 x^{3}-0.5075 x^{4}\right)
$$

where $h$ is the maximum thickness of the airfoil. The profile of the NACA 0012 airfoil is presented in Fig. $6 \mathrm{a}$. The results are given in Fig. $6 \mathrm{~b}$ in terms of the scaled thickness parameter, $\tilde{h}$. This airfoil has a wedgeshaped trailing edge and is free of inflection points and therefore is expected to have unique behavior of the separation point, $x_{o}$, versus the airfoil thickness parameter, similar to the behavior sketched in Fig. 1. As seen in Fig. 6b, unique behavior is indeed predicted. As the airfoil thickness decreases, the separation point moves aft toward the trailing edge. The curve terminates at the value of $\widetilde{h}$ (equal $\widetilde{h}_{c}$ ) which corresponds to the collapse of the free streamline to the $x$-axis at downstream infinity (cut-off). Cut-off is determined to have occurred when the coefficient of the parabolic term in the downstream free streamline shape, ( $b$ in Eq. $(10)$ ), is equal to zero; for this airfoil cut-off occurs at a value of $\widetilde{h}$ equal to 0.093758 for which $x_{0}$ is equal to 0.64297 . Any further decrease of the airfoil thickness parameter causes the free streamline to cross the $x$-axis at a finite distance downstream, which is not a physically plausible solution. For thicknesses below $\widetilde{h}_{c}$, an analysis which accounts for the finite eddy length must be employed, such as that used by Rothmayer (Ref. 22).

The second case considered is the family of airfoils obtained by linearly scaling the profile of the uncambered Garabedian and Korn (GK) 70-10-13 airfoil (Ref. 33). The notation GK 70-10-XX as used here refers 
to a symmetric airfoil with percentage $\mathrm{XX}$ thickness to chord ratio. The equation describing this airfoil, obtained by performing a least-squares fit of the discrete data given in Ref. 33, is given by

$$
f(x)=h\left(1.42384 \sqrt{x}-0.800726 x+0.141386 x^{2}-2.49882 x^{3}+1.73432 x^{4}\right)
$$

The profile of the 13 percent thick airfoil shown in Fig. 7a displays the presence of an inflection point near the trailing edge. The existance of an inflection point introduces the possibility of non-uniqueness in the location of the separation point for a given value of the thickness parameter (Refs. 17 and 22), as illustrated in Fig. 2. Nonuniqueness has been found to occur for the GK airfoil, for which the separation point behavior as a function of the airfoil thickness parameter is shown in Fig. $7 \mathrm{~b}$. The nonunique behavior is not obvious on the scale of Fig. $7 \mathrm{~b}$, but becomes apparent in the expanded view shown in Fig. 7c, which illustrates the behavior of the separation point location near the cut-off value of $\widetilde{h}$. For this airfoil the value of $\widetilde{h}$ at cut-off is equal to 0.071946 and the corresponding location of the separation point, $x_{o}$, is equal to 0.60074 .

The way in which non-unique behavior is manifested for those airfoils in which it occurs can vary significantly. In order to illustrate this point, a third airfoil has been analyzed using the triple-deck analysis; this airfoil was not analyzed using the interacting boundary-layer procedure. The airfoil considered is a symmetric NACA 6-series airfoil; the NACA 65-010 thickness distribution given as discrete points in Ref. 22 was fit using a least-squares curve fit to obtain the polynomial equation

$$
f(x)=h\left(1.10714 \sqrt{x}-0.64158 x+2.11311 x^{2}-5.61493 x^{3}+3.03386 x^{4}\right)
$$

This expression was scaled to obtain a family of airfoils related to the NACA 65-010 airfoil by the thickness parameter, $\widetilde{h}$. Although it is not formally correct to refer to these as NACA $65-0 \mathrm{XX}$ airfoils, since different thickness distributions in the NACA 6-series are not obtained by direct scaling, the XX notation will be used here for convenience.

The profile of the 10 percent thick airfoil is shown in Fig. 8a, and the behavior of the separation point as the thickness parameter is varied is shown in Fig. $8 \mathrm{~b}$. The nonuniqueness is much more pronounced in this case than in the case of the GK 70-10-XX airfoil. In particular, instead of the nonunique behavior occurring when the separation point is in the vicinity of the trailing edge, it occurs as the separation point approaches the leading edge. In addition, the curve is seen in Fig. 8c, which is a detail of Fig. 8b near $x_{0}=0$, to reverse direction as the separation point moves very close to the leading edge. Fig. $8 \mathrm{c}$ shows that, for this airfoil geometry, the separation point moves infinitesimally close to the leading edge as the thickness parameter is increased. Eventually, as the airfoil thickness increases, consequently increasing the perturbation which the airfoil induces upon the global pressure field, the separation point will retreat away from the leading edge as the fixed magnitude $\left(O\left(R e^{-1 / 16}\right)\right)$ perturbation to the global pressure field due to the local triple-deck structure shrinks relative to the perturbation due to the airfoil and free streamline; the retreat from the leading edge occurs when the unscaled airfoil thickness is $O\left(R e^{-1 / 17}\right)$ (see Ref. 17). For the NACA 65-0XX airfoil the cut-off value of $\widetilde{h}$ is equal to 0.059234 , for which the corresponding value of $x_{o}$ is equal to 0.64855 .

Based upon the results displayed in Figs. $8 \mathrm{~b}$ and $8 \mathrm{c}$, the NACA $65-0 \mathrm{XX}$ airfoil is concluded to be a geometry which shows a clear possibility for a hysteresis loop similar to that sketched in Fig. 2.

A simple tool for determining the behavior of the separation point at the asymptotic condition of infinite Reynolds number, when the inviscid flow is assumed to be governed by the thin airfoil equations and the separation point behavior is assumed to be that determined by triple-deck theory, has been demonstrated for three realistic symmetric airfoil geometries. The existence of different types of behavior, which are dependent upon the particular airfoil geometry and include the possibilities of nonuniqueness and hysteresis, has been illustrated.

\section{Interacting-Boundary Layer Analysis}

The Interacting Boundary-Layer (IBL) analysis described in the previous section has been used to predict the way in which the flow undergoes crossover from local to massive separation, and to study the effects upon crossover of turbulence and the application of boundary-layer control.

The IBL calculations have been performed in two modes; the first mode is referred to as the "local stronginteraction" (LSI) mode and the second is referred to as the "massive separation" (MS) mode. In the local 
strong-interaction mode, cases with strong viscous-inviscid interaction, where the flow is either attached or separated locally (on the airfoil or in the vicinity of the trailing edge), are treated. In the massive separation mode the flow is assumed to have undergone crossover to a large-scale separated structure which is modeled using the Kirchhoff eddy model.

Both IBL calculation modes were verified prior to embarking upon the in-depth study discussed below. The LSI mode was first verified by calculating the flow over two configurations which have been analyzed in numerous previous studies. These two configurations are the finite flat plate, which has a local region of strong interaction in the neighborhood of the trailing edge, and the so-called Carter-Wornom trough (Ref. 34), which is essentially a depression on a flat plate which, under the appropriate conditions of Reynolds number and depth of the depression, produces a confined region of separated flow. Although not presented here, the results of the calculations performed for these geometries were in very good agreement with the results of other investigators who have considered these problems. The MS mode was not as easily verified, since very few calculations of this type have been performed to date. However the basic procedure was verified by comparing with results obtained previously by Rothmayer (Ref. 22). Agreement with those results, obtained using a very similar procedure to that employed here, was found to be excellent.

All of the airfoil results discussed below have been obtained using the blunt leading-edge form of the interaction procedures presented in the last section.

Two of the realistic airfoil geometries considered in the infinite Reynolds number (triple-deck) asymptotic analysis and discussed above have been analyzed using the finite Reynolds number (IBL) analysis, namely, the NACA 00XX and the Garabedian and Korn 70-10-XX airfoils. Both the local strong-interaction and the massive separation modes have been utilized to study the process by which a local separation bubble undergoes crossover to a massively separated eddy as the airfoil thickness is increased. In addition to the basic crossover analysis, the impact of turbulence and boundary-layer control upon flow with largescale separation is studied for the NACA 00XX airfoil. Unless otherwise noted, the calculations have been performed assuming laminar flow at a Reynolds number of $10^{6}$.

\section{NACA 00XX Airfoil Series}

The first airfoil which is considered is the NACA 00XX family described by Eq. (64), where the parameter $h$ represents the maximum airfoil thickness to chord ratio. Calculations have been performed using the LSI analysis with the airfoil thickness systematically increased until the approach to cut-off (wake inflation) is observed. Following wake inflation, the MS analysis is applied as the airfoil thickness is further increased.

The predicted behavior of the separation and reattachment points, $x_{s}$ and $x_{r}$, is shown in Fig. 9 as the airfoil thickness is varied. Starting with a one percent thick airfoil, $h$ is increased until incipient separation is observed at the trailing edge when $h=0.03$. Further increasing the airfoil thickness results in growth of the separation bubble about the trailing edge. As the thickness increases, the separation point migrates upstream towards the leading edge while the reattachment point retreats from the trailing edge at an increasing rate. The approach of the curve of the reattachment point versus $h$ to a vertical tangent signals the onset of wake inflation and the crossover from local to massive separation. In the present case, crossover occurs for a value of $h$ between 0.06 and 0.07 . For values of $h$ beyond 0.06 , the massive separation analysis has been applied. The results obtained using the MS analysis show that the separation point continues to move towards the leading edge as $h$ increases. Of particular importance to note in Fig. 9 is the apparently smooth merging of the behavior of the separation point in going from the local strong-interaction to the massive separation model, which is a manifestation of the continuous crossover from the local separation to the massive separation flow structure which is predicted by triple-deck theory for airfoil geometries free of inflection points, and having a wedge-shaped trailing edge.

The effects of varying the airfoil thickness upon the displacement body and the local aerodynamic properties, namely, $C_{p}$, the pressure coefficient, and $C_{f}$, the skin friction coefficient, are illustrated for the NACA $00 X X$ airfoil family in Figs. 10a-d. The skin friction coefficient is defined according to the relation

$$
C_{f}=\sqrt{R e} \frac{\left(\mu^{*} \frac{\partial u^{*}}{\partial n^{*}}\right)_{w a l l}}{\frac{1}{2} \rho_{\infty}^{*} U_{\infty}^{* 2}}=\frac{2 U_{e_{o}}^{2}}{\sqrt{2 \xi}} F_{\eta}(\xi, 0)
$$


In Fig. 10a the airfoil profiles and the associated displacement bodies are plotted for various values of the thickness parameter, $h$. The location of the separation point is also noted in each case. Recall that the results shown were obtained with the LSI analysis for $h \leq 0.06$ and with the MS analysis for $h>0.06$. First, note that as $h$ increases the displacement thickness increases, particularly in the vicinity of the trailing edge and in the downstream wake. As the value of $h$ increases through wake inflation $(0.06<h<0.07)$, the displacement body in the vicinity of the airfoil does not undergo any dramatic change in behavior. However, the length of the separated region has undergone a dramatic change after inflation, extending downstream to a distance on the order of the flow Reynolds number (according to the asymptotic theory), instead of an $O(1)$ distance prior to the onset of wake inflation (see Ref. 17). Another observation to be made with reference to Fig. 10a is the clear tendency of the displacement body towards parabolic growth downstream, seen especially for the NACA 0010 airfoil.

Figures $10 \mathrm{~b}$ and $10 \mathrm{c}$ illustrate the behavior of the pressure coefficient, $C_{p}$, as the airfoil thickness is varied. In Fig. 10b the pressure distributions for the calculations obtained using the LSI approach are shown along the airfoil and downstream to a distance of one chord length beyond the trailing edge. The development of a distinct pressure plateau is seen as the airfoil thickness increases to a value of 0.06 . Recall that the existance of a constant pressure region downstream of the separation point is the fundamental feature assumed in applying the Kirchhoff eddy model to the massive separation calculations; the emergence of a constant pressure region as the separation bubble grows is clearly demonstrated in the results obtained using the local strong-interaction procedure.

In Fig. 10c the pressure distributions are shown on the airfoil surface for both the local strong-interaction and massive separation calculations. As the airfoil thickness increases, the pressure coefficient distribution is observed to change in a smooth manner as the separated region undergoes wake inflation. The continuous change in character of the pressure distribution as the airfoil thickness increases through crossover is consistent with the smoothly varying growth of the displacement body seen in Fig. 8a when crossover occurs. The kink in the pressure distribution seen for some of the LSI results in the vicinity of $x=0.2$ is associated with the use of a numerical grid which is relatively coarse in that region, which was confirmed by performing a calculation which had a significantly finer grid in that region and for which a similar kink was not observed. The use of a coarse grid locally is dictated by the need for a very fine mesh near the leading and trailing edges, and the necessity that the total number of grid points be limited to a reasonable number, which results from the desire to keep computer storage and run times within reasonable limits.

The behavior of the skin friction coefficient, $C_{f}$, is shown in Fig. 10d. As noted for the pressure coefficient, the behavior of the skin friction coefficient distribution changes smoothly as the flow undergoes crossover from local to massive separation with an increase in airfoil thickness. The flow is attached along the entire airfoil for thicknesses below $h=0.03$. At $h=0.03$ incipient separation is observed at the trailing edge while above this thickness the separation bubble grows about the trailing edge until its length approaches 60 percent of chord when $h$ is equal to 0.06 . Massive separation is predicted for $h>0.06$ with the skin friction very close to zero throughout most of the separated region. Consistent with the nearly zero skin friction, the reversed flow velocities were found to be quite small in the separation bubble. This result is expected since the eddy is a nearly stagnant flow region in the Kirchhoff model.

\section{Drag Results}

An important parameter which is generally desired from airfoil calculations is the drag. The nondimensional drag coefficient is given by the expression

$$
C_{D}=\frac{D^{*}}{\frac{1}{2} \rho_{\infty}^{*} U_{\infty}^{* 2}}
$$

where $D^{*}$ is the dimensional drag force. The total drag coefficient, $C_{D}$, is the sum of two components, $C_{D_{p}}$, the drag due to pressure, and $C_{D_{f}}$, the drag due to the skin friction. The (dimensional) pressure drag can be written for a symmetric airfoil at zero angle of attack in the form

$$
D_{p}^{*}=2 \int_{0}^{1} p^{*} \frac{d f^{*}}{d x^{*}} d x^{*}
$$


where the pressure is evaluated at the airfoil surface and the airfoil geometry is given by the expression $y^{*}-f^{*}\left(x^{*}\right)=0$. An asterisk denotes a dimensional variable. The skin-friction drag is obtained from the following equation,

$$
D_{f}^{*}=2 \int_{0}^{1} \tau^{*}\left(x^{*}\right) d x^{*}
$$

where $\tau^{*}$ is the wall shear, $\mu^{*} \partial u^{*} / \partial n^{*}$. The airfoils are assumed to span $x=0$ to $x=1$ in writing Eqs. (69) and (70). These expressions are rewritten in terms of the appropriate nondimensional variables and numerically integrated using trapezoidal rule integration to obtain the final values of the components of the drag coefficient and the total drag coefficient.

Before discussing the particular behavior observed for the drag calculated for the NACA 00XX airfoil family, some remarks regarding the observed behavior of the drag coefficient as a function of the location chosen for the downstream boundary of the strong-interaction region in the LSI calculations are appropriate.

The downstream boundary location has been found to have a strong influence on the value of the drag which results from the numerical calculations. Downstream of the strong-interaction region, the displacement thickness is assumed to decay like $x^{-1 / 2}$; this asymptotic behavior is incorporated into the LSI calculation procedure to account for the effect of the displacement body distribution in the region outside of the calculation domain on the flow in the strong-interaction region. The LSI calculations were initially performed with the downstream boundary located one chord length aft of the trailing edge of the airfoil. The resulting trend in the drag coefficient as the airfoil thickness was varied was observed to be counter to the behavior which is expected. The mechanism which was found to be responsible for the anomalous behavior is the placement of the downstream boundary too close to the trailing edge of the airfoil.

The observation noted above leads to the conclusion that the drag, which is a global quantity, provides a useful parameter in the local strong-interaction calculations for determining when the downstream boundary has been located far enough away from the airfoil. In the present calculations, inspection of the local quantities such as pressure and skin friction gave no indication that the downstream boundary was placed too close to the airfoil.

The behavior of the drag obtained from the study of the NACA 00XX series airfoil is shown in Fig. 11. First note that at $h=0$, the flat-plate drag coefficient determined by Jobe and Burggraf (Ref. 35) using triple-deck theory is indicated. The remaining points are the numerically determined values obtained in the present study using the interacting boundary-layer approach. The drag coefficient is observed to approach the asymptotic flat-plate value very well as the thickness decreases towards zero. The drag increases with increasing airfoil thickness, doing so more rapidly as the thickness becomes larger. The contribution to the total drag from the skin-friction is nearly a constant as the thickness varies, while almost the entire change in the total drag is attributable to the variation in the pressure drag.

The curves of $C_{D}$ and $C_{D_{p}}$ versus $h$ are seen to be discontinuous in going from the LSI to the MS results between $h$ equal to 0.06 and $h$ equal to 0.07 . Based upon the continuous behavior of the separation point in going from the local to the massive separation structure, this discontinuity is unexpected. A strong clue to the cause of this behavior is provided by the fact that the behavior of the friction drag component is continuous as the crossover from local to massive separation occurs while the pressure drag component is discontinuous. The pressure distribution illustrated for the NACA 0006 airfoil in Fig. 10b shows that the level of the plateau pressure is higher than the freestream pressure. If the constant pressure in the eddy of the massively separated flow is in reality higher than the freestream value, the pressure drag would be higher than that predicted using the Kirchhoff eddy model, wherein the eddy pressure has been assumed to be equal to the freestream pressure. Thus, there is a strong possibility that the discontinuity in the behavior of the drag in switching from the LSI to the MS analysis is due to the assumption that the eddy pressure is equal to the freestream pressure, which gives no net contribution to the pressure drag from the separated flow region.

The broader conclusion with regard to the aforementioned anomalous behavior of the drag coefficient is that it is indicative of a deficiency in the Kirchhoff eddy model, which is too restrictive because of the requirement that the eddy pressure be imposed, instead of being obtained as part of the overall solution. Furthermore, the assumption of a constant eddy pressure is probably too restrictive; one would most likely have to allow for variations in the pressure along the eddy in order to develop a more realistic model for 
massively separated flow. Although the local structure in the vicinity of the body has been postulated to be close to that of a Kirchhoff eddy, the modifications of the body scale flow due to the larger scale flow encompassing the global eddy structure will most likely have to be accounted for in order to get a completely self-consistent model of the massively separated flow structure (Ref. 14).

It is expected that, as the Reynolds number for an interacting boundary-layer calculation is increased, the finite Reynolds number result should approach the infinite Reynolds number (triple-deck) result. All of the finite Reynolds number results presented above were calculated at a Reynolds number of $10^{6}$. In order to confirm that the asymptotic behavior is approached as the Reynolds number increases, further calculations have been performed for the NACA 00XX-series airfoil at a Reynolds number of $10^{7}$ using the MS analysis. In order to show the trend in the behavior, the finite Reynolds number results have been obtained over a limited range of airfoil thicknesses; it is not intended here to show the entire range of behavior for each Reynolds number.

The behavior of the separation point for the two finite Reynolds numbers, as well as the prediction obtained from the asymptotic analysis, is presented in Fig. 12, where the airfoil thickness is expressed in terms of the scaled parameter, $\widetilde{h}$ (Eq. (63)), to permit comparison with the behavior predicted by triple-deck theory. As the Reynolds number is increased, the locus of the separation point locations calculated using the finite Reynolds number MS approach is observed to tend towards the asymptotic result. However, it is obvious that convergence to the limiting asymptotic behavior as Re increases occurs very slowly. The slow approach to the asymptotic prediction for the behavior of the separation point is in contrast to the excellent agreement of the predictions of the finite Reynolds number LSI calculations with the result of the asymptotic theory for the flat-plate drag illustrated in Fig. 11. In the theory which applies to the latter case, the small parameter in the asymptotic expansion is $O\left(R e^{-1 / 2}\right)$, whereas in the asymptotic theory governing cut-off the small parameter is $O\left(R e^{-1 / 16}\right)$. The latter parameter approaches zero considerably more slowly than the former as $R e \rightarrow \infty$, which explains the much slower approach of the finite $R e$ cut-off behavior as comparec to the approach of the finite Reynolds number drag behavior to the asymptotic limit as the Reynolds numbes increases. Slow convergence of the finite Re results for cut-off to the predicted asymptotic behavior was alsc observed in Ref. 22.

\section{Garabedian and Korn 70-10-XX Airfoil Series}

The aerodynamic properties of the NACA 00XX series airfoil were expected, based upon triple-deck theory, to change in a continuous fashion as the airfoil thickness was increased through the critical value associated with wake inflation. The IBL analysis for that airfoil at a Reynolds number of $10^{6}$ yielded results which are generally consistent with the predictions of the asymptotic theory. The possibility of behavior which is not continuous (due to non-uniqueness and/or hysteresis) as the airfoil thickness increases through cut-off for certain types of airfoil profiles has been discussed in the previous section. This possibility has been shown to exist for an airfoil with an inflection point in the vicinity of the trailing edge (Ref. 22). In order to determine whether discontinuous behavior occurs at finite Reynolds numbers for a realistic airfoil, the scaled thickness distribution associated with the Garabedian and Korn (GK) 70-10-13 airfoil (Ref. 33), for which discontinuous behavior during crossover was predicted by the triple-deck analysis, has been considered. The airfoil is described by Eq. (65). The family of GK 70-10-XX airfoils was obtained by varying the coefficient $h$ in Eq. (65), where $h$ is the airfoil thickness to chord ratio. The interacting boundary-layer analysis was carried out in the same manner as described above for the NACA 00XX series, and the results are presented below.

The behavior of the separation and reattachment points, $x_{s}$ and $x_{r}$, is shown in Fig. 13. First consider the behavior calculated using the local strong-interaction analysis as the thickness is increased from an initially small value. The boundary layer remains attached to the airfoil until separation is first observed for $h$ equal to 0.05 . Note that initially the entire separation bubble forms upstream of the trailing edge, which is due to the inflection point in the airfoil profile ahead of the trailing edge. The inflection point induces an adverse pressure gradient locally which in turn provokes separation when the airfoil thickness becomes sufficiently large. The mechanism which induces separation in the presence of a geometrical inflection point is different than the mechanism which is present when the airfoil closes in a wedge-shaped trailing edge, where a strong singularity in the inviscid flow is present, which induces separation at the trailing edge. 
Once separation occurs on the GK airfoil, a very small increase in the airfoil thickness causes a considerable expansion of the the separation bubble in the streamwise direction, in contrast to the slow initial growth of the separated flow region which is observed for the NACA 00XX airfoil after the boundary layer first separates. In particular, in Fig. 13 note the extremely large downstream motion of the eddy closure point which occurs between values of $h$ equal to 0.052 and 0.053 for the GK airfoil.

Calculations have also been performed using the massive separation analysis starting with a 10 percent thick airfoil and decreasing the airfoil thickness to a minimum value of $h$ equal to 0.057 . Below this value the MS results indicate that the eddy has collapsed to the $x$-axis at a finite distance downstream of the trailing edge; i.e., cut-off has occurred.

The curves of $x_{s}$ versus $h$ obtained from the LSI and MS analyses and presented in Fig. 13 do not merge smoothly as cut-off is approached, in contrast with the smooth behavior observed previously for the NACA 00XX airfoil (cf. Fig. 9). The contrast between the behavior predicted for the NACA 00XX and the GK 70-10-XX airfoils by the finite Reynolds number analysis appears to be consistent with the contrast between the predictions of the asymptotic analysis, although it is unclear from these results precisely how the behavior predicted for the GK 70-10-XX airfoil by the triple-deck and IBL approaches is related (cf. Figs. 7 and 13). In any event, the lack of smooth merging of the results of the finite Reynolds number LSI and MS analyses appears to be related to the presence of an inflection point in the GK airfoil geometry, and is therefore consistent the prediction of triple-deck theory that airfoils which have inflectional profiles like that of the GK thickness distribution are not anticipated to undergo continuous crossover between local and massive separation (Refs. 17 and 22).

The effects of varying the airfoil thickness upon the computed displacement body distributions and local aerodynamic properties, namely the pressure coefficient and skin friction coefficient, calculated for the GK airfoil series are shown in Figs. 14a-c, respectively.

The GK airfoil profiles and the associated displacement bodies are shown in Fig. 14a, where the separation points are also indicated. Several observations are made based upon this figure. First, the displacement thickness at a given streamwise location in the downstream wake is seen to be nearly constant as $h$ increases through a value of 0.05 . However, the small increase in airfoil thickness from 0.050 to 0.053 is seen to provoke a very large change in the displacement thickness distribution in the wake and on the aft portion of the airfoil, which is associated with the enormous expansion of the separation bubble occurring between those two values of $h$ (see Fig. 13). Increasing the thickness of the airfoil from 0.053 to 0.057 , which requires switching from the local strong-interaction to the massive separation analysis, does not show the same rapid inflation of the eddy which is observed in going from a value of $h$ equal to 0.050 to 0.053 . The discontinuity in the rate of inflation of the eddy may be a further indication of the lack of continuous merging of the local and massive separation flow structures as the airfoil thickness increases through cut-off. Once the flow has crossed over to a massively separated state, further increasing the airfoil thickness leads to a smoothly increasing displacement body thickness downstream of separation.

In Fig. 14b the distribution of the pressure coefficient on the airfoil surface is shown for the various values of $h$ considered. Consistent with the increase in the extent of separation going from $h$ equal to 0.050 to 0.053 , a significant pressure plateau is observed in the latter case. The pressure distribution obtained from the massive separation calculation for a value of $h$ equal to 0.057 is very close to that for $h$ equal to 0.053 from the LSI analysis, with about the same extent predicted for the pressure plateau on the airfoil surface, which is consistent with the similar displacement body shapes predicted for these two cases. Increasing the airfoil thickness above 0.057 leads expansion of the pressure plateau upstream, consistent with the upstream movement of the separation point.

The skin friction distributions for the GK airfoil are presented in Fig. 14c. The approach to separation is observed, with a small separation bubble initially forming ahead of the trailing edge for the case of $h$ equal to 0.05 . The rapid upstream movement of the separation point as the thickness increases to 0.053 is clearly seen in this figure, as is the continuing tendency of the separation point to move further upstream as $h$ continues to increase.

\section{Turbulence Effects}

The effect of turbulence upon the onset of massive separation has been studied as part of the present 
effort. Turbulence is simulated through the use of an algebraic turbulence model, which is described in Appendix B. Two geometries are considered, namely, the NACA 00XX airfoil and a circular cylinder with a trailing edge splitter plate.

\section{NACA 00XX Airfoil}

The NACA 00XX series airfoil has been studied at a Reynolds number of $10^{6}$ in an attempt to calculate the crossover between local and massive separation occurring in the presence of a turbulent boundary layer. Transition to turbulence is assumed to occur instantaneously at a specified location on the airfoil surface. Since the present investigation into the effects of turbulence is only concerned with the qualitative aspects, the transition location has been arbitrarily specified to be at $x=0.35$ for all of the turbulent airfoil calculations.

In the laminar calculations performed in the LSI mode, the downstream wake displacement thickness is assumed to be of the Goldstein form, with $\delta^{*} \sim x^{-1 / 2}$. For a turbulent wake the displacement thickness also decays like $x^{-1 / 2}$, but the constant 0.664 in the Goldstein form (Appendix E, Eq. (E.9a)) is dependent upon the upstream solution; this issue is discussed in greater detail in Appendix E.

The present study of the effect of turbulence has been performed with the Reynolds number and transition location fixed at the values stated above, and the airfoil thickness to chord ratio has been varied up to a maximum value of 14 percent. At the maximum thickness the streamwise extent of the separation bubble predicted using the LSI analysis is less than 1 percent of chord, which is in contrast with the result of the calculation for laminar flow past a 6 percent thick airfoil, wherein a separation bubble with a length equal to approximately 60 percent of the airfoil chord has been predicted. For otherwise similar flow conditions, turbulence is known to suppress the onset of separation, forcing it to initially occur for a much more severe geometry (i.e., thickness) than when the flow is laminar. The present results serve to indicate the degree to which separation can be suppressed by turbulence.

Recall that the triple-deck analysis for laminar flow results in a thickness parameter, $\tilde{h}$, defined by Eq. (63), which involves the Reynolds number. Increasing $\widetilde{h}$ is equivalent to either increasing $h$ at a fixed value of $R e$ or increasing $R e$ at a fixed value of $h$. Massive separation can therefore be provoked, in laminar flow, by either increasing the airfoil thickness, which the bulk of this study has been concerned with, or by increasing the Reynolds number. Based upon this observation, the latter approach has been considered in an effort to obtain a massively separated turbulent flow solution for a reasonable value of the airfoil thickness.

In Fig. 15, the locations of the separation and reattachment points are shown as a function of the Reynolds number for the NACA 0014 airfoil, where instantaneous transition to turbulence has been imposed at $x=0.35$. It is observed that the separation bubble decreases in streamwise extent as the Reynolds number increases for a fixed value of $h$; the bubble vanishes between $R e$ equal to 1 and 2 million. The decreasing extent of the separation bubble in turbulent flow is in opposition to the trend predicted for laminar flow by triple-deck theory, as well as being opposite to the trend observed at finite Reynolds number using the present interacting boundary-layer approach for laminar flow (e.g., see Fig. 9). Of course, whether the flow is laminar or turbulent, increasing the airfoil thickness at a fixed value of the Reynolds number increases the streamwise extent of separation. The observed opposing trend in the behavior of the extent of separation as a function of the Reynolds number between laminar and turbulent flow is evidence that there must be a fundamental difference between the two types of flow in the local structure which arises in the vicinity of the separation point, which is not surprising. At the present time the nature of the local structure of a turbulent flow in the vicinity of separation is not well understood; until it is, the precise reason for the opposing trend in behavior discussed above will remain unexplained.

\section{Circular Cylinder}

No massively separated turbulent flow solutions have been obtained for the NACA 00XX series airfoil over the range of the thickness parameter considered. However, the expectation is that by increasing the airfoil thickness until it is $O(1)$, that is, until the airfoil becomes a bluff body, massively separated solutions should result. In order to demonstrate that a massively separated solution can be computed for a turbulent flow, instead of considering bluff airfoils, the transitional flow past a circular cylinder geometry has been calculated herein. The specific case considered is the flow past a circular cylinder with a downstream splitter 
plate, at a Reynolds number (based on diameter) of 14,500 , for which experimental data is available (Ref. 36). A configuration with a splitter plate has been chosen since the splitter plate suppresses the large scale unsteadiness associated with vortex shedding, hence making the experimental data more suitable for comparison with the present steady-state numerical results.

The massive eddy which is present in a bluff-body flow is generally observed in experiments to be very nearly a constant pressure region, where the pressure level is a function of the geometry and flow conditions. In the present massive separation model, the level of the pressure in the Kirchhoff eddy is a parameter which can be set, however it can not be determined as part of the solution. Therefore, the results of the circular cylinder calculations which are presented below have been obtained by specifying the eddy pressure to be the value measured experimentally; the specified value of the pressure coefficient is $C_{p}=-0.4465$.

An initial calculation has been performed assuming the flow to be completely laminar; the result of this calculation has then been used to determine the transition location for a subsequent turbulent calculation. The transitional calculation has been performed assuming that transition occurs instantaneously just aft of the location at which separation is predicted in the laminar calculation, which occurs at an angular distance of approximately 78.5 degrees around the cylinder measured from the forward stagnation point. The results of both calculations are presented in Fig. 16 along with the experimental data of Roshko (Ref. 36). Massive separation is predicted for both the laminar and the transitional flow. The two results are virtually identical until a value of $\theta$ of approximately 45 degrees, after which a slightly lower pressure is predicted for the turbulent flow. Both pressure distributions agree reasonably well with the experimental data. Note that good agreement with the experiment is obtained despite the fact that the present bluff-body calculations violate the restriction of the present thin-airfoil approach with respect to the ratio $\xi_{o} / r_{o}$, which should be large, as noted earlier, but is $O(1)$ in this case.

Several important conclusions are drawn from the results of the above calculations. The first conclusion is that the presence of a turbulent boundary layer, although it inhibits the initial onset of massive separation, does not prevent the occurrence of large-scale separated flow. Also, results have been obtained which demonstrate that it is possible to compute bluff-body massive separation with the present IBL approach for massively separated flow, however, the eddy pressure cannot be obtained as part of the solution, but must be specified in order to obtain reasonable results. Finally, with the correctly specified value of the eddy pressure, reasonable agreement with experimental data can be obtained despite the violation of the restriction with respect to the ratio $\xi_{o} / r_{o}$.

\section{Boundary-Layer Control}

The reason for studying massive separation is not only to satisfy our curiosity about this intriguing fluid dynamic phenomenon, but also to gain an understanding which will lead to our ability to control and ultimately to eliminate its occurrence. For the latter reason, a brief investigation into the impact of two forms of boundary-layer control, surface suction and tangential injection, upon the onset of massive separation in laminar flow has been undertaken as part of the present study.

\section{Surface Suction}

For the past decade there has been a considerable amount of research and development effort into the use of surface suction to maintain laminar flow over airfoils in order to improve their efficiency. The primary mechanism by which efficiency is improved is through the drag reduction achieved by maintaining a laminar boundary layer over as much of the wing as is possible, and the goal is generally to do so under cruise conditions. In the present study, the application of suction is instead considered under the far from optimal conditions associated with the onset of massive separation. The intent is to determine whether an airfoil which would ordinarily be stalled without the application of surface suction would remain so in the presence of mild surface suction, and to demonstrate a preliminary capability for the analysis of techniques for the control of boundary-layer separation.

The airfoil for which this analysis has been undertaken is the NACA 00XX airfoil, over which laminar flow is assumed, and the Reynolds number is taken to be $10^{6}$. All of the suction and injection calculations have been performed with the local strong-interaction analysis. Without suction, the crossover to massive 
separation had been found to occur in the thickness range between 6 percent and 7 percent. With this in mind, the 6 percent thick airfoil, NACA 0006, is considered first. The distribution of the surface normal injection velocity is assumed to be sinusoidal and is expressed in terms of the physical velocity as

$$
v_{w}(x)= \begin{cases}R e^{1 / 2} v_{w}(x)^{*} / U_{\infty}^{*}=a_{w} \sin \left(\pi \frac{x-x_{1}}{x_{2}-x_{1}}\right), & x_{1}<x<x_{2} \\ 0, & x \leq x_{1} \text { and } x \geq x_{2}\end{cases}
$$

where $a_{w}$ is a parameter which determines the magnitude of the normal injection and $x$ is the distance along the chord line ( $x$-axis) from the leading edge. The quantities $x_{1}$ and $x_{2}$ denote the starting and ending locations of the suction distribution; outside of the region bounded by $x_{1}$ and $x_{2}$ no suction is applied. Note that when $a_{w}$ is negative, suction is applied as opposed to injection when $a_{w}$ is positive. Also note that the magnitude of the dimensional normal velocity, $v_{w}^{*}$, is small, being of the same order of magnitude of the normal velocity within the boundary layer, $O(1 / \sqrt{R e})$. Expressed in terms of the Görtler variable for the normal velocity, the surface suction velocity is given by

$$
V_{w}=\frac{\sqrt{2 \xi}}{U_{e_{o}}} v_{w}
$$

The first case considered is for suction applied along the entire chord of the airfoil, i.e., $x_{1}=0$ and $x_{2}=1$. Two non-zero magnitudes for the suction parameter have been considered: $a_{w}=-0.5$ and $a_{w}=-1.0$. The suction distributions are presented in Fig. 17. The results for these two cases are compared with the results for the baseline case without suction in Figs. 18a-d.

The airfoil and the displacement body for the three cases are presented in Fig. 18a, along with the location of the separation point. A dramatic change is induced in the displacement body, particularly in the vicinity of the trailing edge, by the application of a small amount of surface suction applied over the full chord of the airfoil. Figs. $18 \mathrm{~b}$ and $18 \mathrm{c}$ show the surface pressure and skin friction distributions obtained for the corresponding surface suction distributions. The application of surface suction is seen in Fig. 18b to cause a dramatic decrease in the extent of the pressure plateau present in the trailing-edge region of the airfoil without suction, for which there is an extensive separation bubble. An increase in the amount of suction results in an increase in the peak pressure at the trailing edge and a further reduction in the extent of the pressure plateau, with the plateau almost entirely eliminated when $a_{w}=-1.0$. The pressure distribution is observed to become more "inviscid-like" (i.e., more singular in nature) as the displacement body becomes thinner with the increasing amount of suction. The skin friction distributions are seen in Fig. 18c to be strongly influenced by the amount of surface suction which is applied, with the skin friction generally increasing as the suction parameter $a_{w}$ increases. Fig. $18 \mathrm{~d}$ shows the behavior of the skin friction in the vicinity of the trailing edge, where the flow is observed to remain separated for both values of the suction parameter, with the length of the separated region decreasing as $a_{w}$ increases.

The total drag coefficient and its individual components are given in Table 2 below.

TABLE 2

Drag coefficients for NACA 0006 with full-chord suction

$\begin{array}{cccc}a_{w} & C_{D} \times 10^{3} & C_{D_{p}} \times 10^{3} & C_{D_{f}} \times 10^{3} \\ & & & \\ 0.0 & 4.654 & 2.354 & 2.300 \\ -0.5 & 4.560 & 1.262 & 3.298 \\ -1.0 & 5.482 & 1.184 & 4.299\end{array}$

The total drag initally undergoes a slight decrease, but then rises as the suction velocity is further increased. The increase in the total drag is clearly seen to be due to the pronounced increase in the friction drag accompanying the increasing suction velocity (Fig. 18c). The decrease in the pressure drag due to the decreasing extent of separation is not sufficient to counteract the increasing friction drag.

The goal of applying boundary-layer control is to improve the performance of the airfoil, which translates in the present study to a reduction in the drag. The use of full-chord suction is observed to be ineffective in 
achieving a significant reduction in the total drag because of the large penalty incurred due to the increase in the friction drag. Therefore, in order to achieve the desired improvement in the airfoil performance, a reduction in the pressure drag component is sought without a concomitant increase in the friction drag. In an attempt to determine if this is possible, additional calculations have been performed with suction applied along a slot which spans a small fraction of the airfoil chord, in contrast to the full-chord suction applied above.

The NACA 0006 airfoil is again considered for the slot suction calculations. The values of $x_{1}$ and $x_{2}$ defining the slot location have been chosen so as to bracket the predicted location of the separation point for the case where no suction is applied, namely, $x_{1}=0.75$ and $x_{2}=0.79$. Two values of the suction parameter $a_{w}$ are utilized, $a_{w}=-1.0$ and $a_{w}=-3.0$. The local distribution of the suction velocity for the two cases is shown in Fig. 19.

The airfoil and displacement body calculated for the slot suction cases are shown in Fig. 20a, along with the displacement body resulting from the calculation without suction. The effect of slot suction upon the displacement body is significant, however the displacement body is not reduced to the extent observed when full-chord suction is applied (cf. Fig. 18a). The pressure distributions are given in Fig. 20b, and show a reduction in the extent of the pressure plateau as the suction parameter is increased, although the plateau remains considerably more pronounced than in the case of full-chord suction (cf. Fig. 18b). The skin friction distributions in the vicinity of the trailing edge are given in Fig. 20c. Upstream of the trailing-edge region the distributions are almost unaffected by the application of slot suction, in contrast to the effect which full-chord suction has on the skin friction distribution over the entire chord of the airfoil. The skin friction initially increases near the slot, followed by a decrease and subsequent separation. The extent of separation is observed to decrease as the suction parameter increases.

In Table 3 below, the total drag coefficient and its individual components are given.

TABLE 3

Drag coefficients for NACA 0006 with slot suction

$\begin{array}{cccc}a_{w} & C_{D} \times 10^{3} & C_{D_{p}} \times 10^{3} & C_{D_{f}} \times 10^{3} \\ & & & \\ 0.0 & 4.654 & 2.354 & 2.300 \\ -1.0 & 3.705 & 1.331 & 2.373 \\ -3.0 & 3.852 & 1.338 & 2.514\end{array}$

As in the case of full-chord suction, the initial decrease in the total drag is followed by an increase as the suction parameter increases, however here the level of the drag is significantly lower than that observed with full-chord suction at comparable values of $a_{w}$. The lower total drag which results when slot suction is applied is due to the significantly smaller increase in the friction drag associated with slot suction as compared to full-chord suction, since in the former case the skin friction only increases locally, near the slot. At the same time, the pressure drag reduction is comparable to that obtained with full-chord suction, leading to an overall decrease in the drag coefficient. The improved drag reduction obtained by application of slot suction as opposed to full-chord suction is achieved with an order of magnitude less mass removed from the boundary layer; for the sinusoidal suction distribution used here, the ratio of mass removal of the full-chord to slot suction case is proportional to the ratio of the chord length to the slot length for a given value of $a_{w}$, which for the aforementioned cases is 25:1.

In order to further demonstrate the applicability of the present boundary-layer control analysis to separated flow problems, a case which has more extensive separation than is predicted for the NACA 0006 airfoil has been studied. The thickness of the NACA 0006 airfoil is below the critical value at which cut-off occurs, so that it is still in the class of locally separated flows, although the separation bubble is an appreciable percentage of the airfoil chord when no suction is applied. A significantly more severe case is represented by the NACA 0007 airfoil, since in this case the flow is predicted by the interacting boundary-layer analysis to be massively separated.

The parameters specified for the NACA 0007 calculation are as follows; the slot endpoints are at $x_{1}=.68$ and $x_{2}=.76$, which places the slot downstream of the separation point determined using the massive 
separation analysis with no suction, and the value of the suction parameter has been set to $a_{w}=-10$. The distribution of the suction velocity is given in Fig. 21. The results of the calculations with and without suction are compared below. The case without suction was calculated using the MS mode and the case with suction was calculated using the LSI mode.

The airfoil and displacement body for the cases with and without suction, along with the location of the separation point, are shown in Fig. 22a. The effect of suction is seen to be dramatic, with the massive separation eddy reduced to a local separation bubble with the application of mild slot suction. As expected, with the suppression of massive separation by the application of suction, the parabolic downstream growth of the displacement thickness which occurs in the massively separated case is no longer observed; instead, the displacement thickness decreases as the flow proceeds downstream.

The pressure and skin friction distributions are shown in Figs. 22b and 22c, respectively, for the NACA 0007 airfoil with and without slot suction. Without suction, the massive separation solution exhibits a pressure plateau extending over approximately the last 30 percent of the airfoil chord (Fig. 22b). The application of suction reduces the pressure plateau considerably and leads to a positive peak pressure in the vicinity of the trailing edge, near the bubble closure point. The skin friction distributions are shown in Fig. 22c. Upstream of aprroximately mid-chord, the skin friction is affected only slightly by the application of the slot suction. In the case without suction, separation occurs at about 60 percent of chord from the leading edge, while it is delayed until approximately 90 percent of chord with suction applied. A large increase in the skin friction is observed in the vicinity of the slot in the latter case, which is followed by a very rapid drop commencing immediately after the slot, and becoming less severe as the separation point is approached.

Because the NACA 0007 airfoil represents a more severe case than the NACA 0006 airfoil, it was found that a larger amount of mass removal from the boundary layer is necessary in the former case to unstall the airfoil and produce a locally separated flow.

The relative effect of the application of suction upon the drag is not presented for the NACA 0007 airfoil because the two calculations were performed using the two different IBL modes, since the case without suction is massively separated and the suction case has a local separation bubble. As discussed earlier with reference to Fig. 11, the drag results obtained using the two methods cannot be compared in a meaningful way unless the appropriate level of the eddy pressure is specified in the massive separation calculation, since the pressure cannot be predicted as part of the present massive separation technique. In the case of the massive separation calculation performed for the NACA 0007 airfoil, the eddy pressure was set to the freestream value, which is probably too low, hence the drag will be underpredicted in this case.

\section{Tangential Injection}

In addition to surface suction, another form of boundary-layer control which is sometimes used is the injection of a tangential jet of air along a portion of the airfoil surface. This technique is intended to take advantage of the so-called Coanda effect, whereby a tangentially injected jet of fluid tends to adhere to a curved surface. This phenomenon has been utilized by injecting air along the curved surface of a flap which is deflected downward. The jet of air and the ambient fluid entrained along with it are guided downward by the surface of the flap, and an additional component of lift is generated. The principal use of this technique has been in applications to short take-off and landing (STOL) aircraft.

Tangential injection like that described above can be simulated, albeit somewhat crudely, by applying a specified slip (or moving wall) boundary condition over some portion of the airfoil surface, which is what has been done in the present study. The boundary condition is modified by replacing the right-hand side of Eq. (26a) with the specified value of $F(\xi, 0)=F_{w}(\xi)$, which is implemented by setting the recursion relation coefficient $Z_{1}$ in Eq. (62a) to $F_{w}$ and the remaining coefficients in Eq. (62a) to zero at the surface $(j=1)$. For this investigation $F_{w}$ is given by

$$
F_{w}(\xi)= \begin{cases}a_{w} \sin \left[\pi\left(1-x / x_{1}\right)\right], & x>x_{1} \\ 0, & x \leq x_{1}\end{cases}
$$

so that the distribution is sinusoidal, being zero upstream of $x_{1}$ and at the trailing edge. This distribution was not chosen with the intention of simulating the distribution of the tangential velocity which might be found in a realistic case, but rather to demonstrate the capability for calculating such cases, and to illustrate 
the effect which tangential injection can have on a configuration for which extensive separation is observed without injection.

The airfoil chosen for this study is again the NACA 0006 airfoil, the Reynolds number is $10^{6}$ and the flow is assumed to be laminar. For the present calculations, $x_{1}$ has been chosen to be 0.75 and the injection parameter $a_{w}$ has been set to three different values, $a_{w}=0.05,0.15$ and 0.50 . The distributions of the surface tangential injection velocity are shown in Fig. 23.

The calculated displacement body is plotted along with the airfoil profile and the separation point in Fig. $24 \mathrm{a}$ for each of the cases. The application of tangential injection is seen to significantly reduce the thickness of the displacement body in the vicinity of the trailing edge. The corresponding pressure distributions are displayed in Fig. 24b. Increasing the injection parameter decreases the extent of the pressure plateau and increases the magnitude of the peak pressure in the trailing edge region. The skin friction distributions are shown in Fig. 24c. The slip condition at the surface causes an increase in the skin friction there. As expected, separation is suppressed by tangential injection.

In Table 4 below, the total drag coefficient and its individual components are given for the cases of tangential injection considered.

TABLE 4

Drag coefficients for NACA 0006 with tangential injection

$\begin{array}{cccc}a_{w} & C_{D} \times 10^{3} & C_{D_{p}} \times 10^{3} & C_{D_{f}} \times 10^{3} \\ & & & \\ 0.0 & 4.654 & 2.354 & 2.300 \\ 0.05 & 4.222 & 1.371 & 2.851 \\ 0.15 & 5.225 & 1.363 & 3.862 \\ 0.50 & 8.425 & 1.309 & 7.117\end{array}$

The pressure drag $C_{D_{p}}$ for all non-zero values of $a_{w}$ is approximately constant, and is considerably less than the friction drag calculated for the baseline case (without injection). The friction drag $C_{D_{J}}$ is affected significantly by the value of the injection parameter, increasing as $a_{w}$ increases. Because of the latter effect, the total drag $C_{D}$ is seen to decrease slightly over the baseline value for the smallest value of the injection parameter, but it is larger than the baseline value for the two largest values of $a_{w}$, since the lower value of $C_{D_{p}}$ cannot compensate for the large increase in $C_{D_{f}}$. 


\section{CONCLUDING REMARKS}

The principal goal of the present study has been to initiate an investigation of the crossover process by which the flow structure evolves from a locally separated to a massively separated flow on realistic airfoil geometries as the airfoil thickness is increased. An understanding of the phenomenon of massive separation is necessary in order to develop techniques for controlling or eliminating massive separation on airfoils in external and internal flow, and thereby improve the performance and extend the safe operating range of aircraft and turbomachinery.

The present analysis of the crossover process has been performed using two methods, one which applies for the asymptotic condition of infinite Reynolds number and utilizes triple-deck theory, and the second which is applicable to finite Reynolds number flow and employs interacting boundary-layer theory. The latter method utilizes two models for the inviscid flow. The first model, which employs the usual Cauchy integral for thin-airfoil theory, is used within the "local strong-interaction" analysis to calculate flows with a localized region of strong viscous-inviscid interaction including flows with a relatively small separation bubble. The second inviscid model, the Cheng-Rott generalized thin-airfoil integral, is utilized in the "massive separation" analysis and is based on the assumption of a constant pressure region downstream of the separation point.

The principal conclusion of the present study is that the interacting boundary-layer approach provides a useful and efficient method for the prediction of separated flows occurring on realistic airfoils. In particular, interacting boundary-layer theory has been demonstrated to be capable of analyzing the crossover process between local, small-scale separation and massive separation, and to thus provide a vehicle for the comprehensive analysis of airfoil aerodynamic performance. The utility of the present approach has been demonstrated by the relative ease with which the effects of turbulence and boundary-layer control on airfoil separation have been incorporated.

The interacting boundary-layer approach utilized in the present study has been developed to provide a reasonable finite Reynolds number theory which is firmly rooted in triple-deck theory, a rational asymptotic theory that applies in the limit of infinite Reynolds number. An important result of the present investigation has been the demonstration that qualitative agreement between the results of the triple-deck and the interacting boundary-layer analyses exists in the predictions for the crossover behavior occurring for realistic airfoils. A consequence of the observed qualitative agreement between the results of triple-deck and interacting boundary-layer theory is the recognition that the asymptotic theory can enhance the understanding of the crossover behavior which is predicted at finite Reynolds number by interacting boundary-layer theory. In addition, triple-deck theory provides the scalings that must be observed in generating mesh distributions for accurate numerical solutions of the finite Reynolds number crossover problem, whether by an interacting boundary-layer approach or a large scale computation using the Navier-Stokes equations, or some intermediate equation set. Since interacting boundary-layer theory provides efficient solutions, even on fine meshes, an analysis like that presented herein can be very helpful in providing guidance in the development of Navier-Stokes solution techniques for the investigation of massive separation and the crossover process, and can provide a basis of comparison for the results of such analyses.

A majority of the present study has been concerned with the crossover from local to massive separation when the flow is laminar. However, since most real boundary-layer flows are turbulent over at least a portion of the airfoil surface, it is important to consider the effect of turbulence upon the crossover process; thus turbulence has been introduced into the present interacting boundary-layer analysis. Turbulence has been verified to strongly suppress the initial onset of separation, thereby delaying stall considerably in comparison with a fully laminar flow. The extent of turbulent separation is observed to decrease as the Reynolds number increases for a fixed airfoil geometry, which is opposite to the trend that is observed for laminar separation.

The present analysis of the crossover process for realistic airfoils has verified the resistance of the boundary-layer to separation in the presence of turbulence. Calculations have been performed which demonstrate, however, that if the airfoil thickness is increased sufficiently, massive separation can occur even if the boundary layer is turbulent. The massively separated flow past a bluff body, namely, a circular cylinder with a trailing-edge splitter plate, has been calculated using the present finite Reynolds number analysis. Both laminar and transitional flow results have been obtained and they both agree reasonably well with the experimentally measured pressure distribution for that configuration, despite the fact that the present interacting boundary-layer analysis was not developed with the intention of being applied to massive separation 
on a bluff-body.

The analysis of the crossover from local to massive separation which has been carried out herein provides a means for better understanding the crossover phenomenon, but more importantly, the present interacting boundary-layer analysis has been demonstrated to be a useful tool with which to study methods for the control and elimination of massive separation. The present interacting boundary-layer analysis has been used to conduct a limited scope study of the effect of boundary-layer control, in the form of suction and tangential injection, upon separated flow and the crossover process. Boundary-layer control techniques have been shown to be effective in suppressing or eliminating both local and massive separation. In particular, the judicious application of slot suction has been shown to yield significant reductions in the drag in cases for which the flow has a large separated region prior to the application of boundary-layer control.

\section{Future Directions}

Several rccommendations for future directions are provided herein, based upon the conclusions of the present study. The present effort has resulted in the development of an efficient analytical tool with which separated flow over airfoils can be analyzed. Further development of various aspects of the present technique would expand the present capability to allow the analysis of a broader range of problems than can currently be addressed, and would permit more accurate modeling of the physical processes occurring in separated flows. Some of the areas which should be considered in future work are discussed below.

The Kirchoff eddy model utilized in the present analysis is a reasonable model for the local body scale flow, but does not provide a means for predicting the constant pressure level in the eddy, which currently has to be assumed or obtained from experimental data. It is evident that a better model for the eddy, in particular, one which would allow the eddy pressure to be determined as part of the solution, should be pursued.

The second suggested area for future study is in the development of a capability to treat lifting airfoils. The present study has been limited to non-lifting airfoils, while in practice massive separation is usually associated with operation of an airfoil at a high angle of attack. The capability to analyze lifting airfoils would make it possible, particularly in conjunction with the development of a better model for the eddy, to address realistic problems in massive separation.

It has been demonstrated that the effects of boundary-layer control can be studied for airfoils up to and beyond separation using the present interacting boundary-layer analysis; therefore, future research into the area of boundary-layer control techniques for the control of separation should exploit the efficient tool which the present approach represents.

The method of analysis which has been developed herein for the study of airfoil separation up to and beyond stall provides the foundation upon which it will be possible to build an analytical tool with the capability to treat increasingly more realistic problems in the future. 


\section{REFERENCES}

1. Cheng, H.K. and Rott, N., Generalizations of the Inversion Formula of Thin Airfoil Theory, J. Rat. Mech. An., Vol. 3, pp. 357-382, 1954.

2. Smith, F.T., The Laminar Separation of an Incompressible Fluid Streaming Past a Smooth Surface, Proc. R. Soc. Lond. A, 356, pp. 443-463, 1977.

3. Rothmayer, A.P. and Davis, R.T., Massive Separation and Dynamic Stall on a Cusped Trailing-Edge Airfoil, 3rd Symposium on Numerical and Physical Aspects of Aerodynamic Flows, Chap. 6, pp. 11-27, 1985.

4. Prandtl, L., Uber Flussigkeitsbewegung sehr Kliener Reibung, Proceedings of the Third International Math. Kongr., Heidelberg, 1904.

5. Neiland, V. Ya., Theory of Laminar Boundary Layer Separation in Supersonic Flow, Azv. Akad. Nauk SSSR, Mekh. Zhid. i Gaza, No. 4, pp. 53-57, 1969.

6. Stewartson, K. and Williams, P.G., Self-Induced Separation, Proc. Roy. Soc. Lond., A312, pp. 181-206, 1969.

7. Messiter, A.F., Boundary Layer Flow Near the Trailing Edge of a Flat Plate, SIAM J. Appl. Math., Vol. 18, No. 1, 1970.

8. Lighthill, M.J., On Boundary Layers and Upstream Influence. II. Supersonic Flows Without Separation, Proc. Roy. Soc. Lond., A217, pp. 478-507, 1953.

9. Sychev, V.V., On Laminar Separation, Mekhanika Zhidkosti i Gaza, No. 3, pp. 47-59, 1972.

10. Kirchhoff, G., Zur Theorie freier Flüssigkeitsstrahlen, J. reine agnew. Math., Vol. 70, 1869.

11. Korolev, G.L., Numerical Solution of Asymptotic Problem on Separating Laminar Boundary Layer at a Smooth Surface, Sci. J. TSAGI, Vol. 11, No. 2, pp. 27-36, 1980.

12. Van Dommelen L.L. and Shen, S. F., Interactive Separation from a Fixed Wall, Proc. 2nd Symp. Numer. Phys. Aspects Aerodyn. Flows, Long Beach, Calif., ed. T. Cebeci, Springer-Verlag, 1984.

13. Smith, F.T., Laminar Flow of an Incompressible Fluid Past a Bluff Body: The Separation, Reattachment, Eddy Properties and Drag, J. Fluid Mech., Vol. 92, Part 1, pp. 171-205, 1979.

14. Smith, F.T., A Structure for Laminar Flow Past a Bluff Body at High Reynolds Number, J. Fluid Mech., Vol. 155, pp. 175-191, 1985.

15. Fornberg, B., Steady Viscous Flow Past a Circular Cylinder up to Reynolds Number 600, J. Computational Physics, Vol. 61, pp. 297-320, 1985.

16. Smith, F.T., On the High Reynolds Number Theory of Laminar Flows, IMA J. of Appl. Mathematics, Vol. 28, pp. 207-281, 1982.

17. Cheng, H.K. and Smith, F.T., The Influence of Airfoil Thickness and Reynolds Number on Separation, Journal of Applied Mathematics and Physics (ZAMP), Vol. 33, March 1982, pp. 151-180.

18. Cheng, H.K., Laminar Separation from Airfoils Beyond Trailing-Edge Stall, AIAA Paper 84-1612, presented at the AIAA 17th Fluid Dynamics, Plasma Dynamics and Lasers Conference, June 25-27, 1984, 
Snowmass, Colorado.

19. Cheng, H.K., Symmetry Breaking and Lift Hysteresis Arising from Massive Laminar Separation: A Study of Multiple Steady-State Solutions, Proc. Conf. Low Reynolds Number Aerodynamics, ed. T.J. Mueller, Univ. Notre Dame, UNDAS-CP-778123, pp. 219-233, June 1985.

20. Rothmayer, A.P. and Smith, F.T., Large-Scale Separation and Hysteresis in Cascades, Proc. R. Soc. Lond. A 402, 83-108, 1985.

21. Woods, L.C., The Theory of Subsonic Plane Flow, University Press, Cambridge, 1961.

22. Rothmayer, A.P., A Study of High Reynolds Number Separation, PhD. Dissertation, University of Cincinnati, Department of Aerospace Engineering and Engineering Mechanics, May 1985.

23. Werle, M.J., and Vatsa, V.N., A New Method for Supersonic Boundary Layer Separations, AIAA J., Vol. 12, No. 11, pp. 1491-1497, Nov. 1974.

24. Carter, J.E., A New Boundary Layer Inviscid Iteration Technique for Separated Flow, AIAA paper 78-1450, 1978.

25. Veldman, A.E.P., A Calculation Method for Incompressible Boundary Layers with Strong Viscous-Inviscid Interaction, NLR MP 79029 U, presented at 3rd GAMM-Conf. on Numer. Meths. in Fluid Mech., Oct. 10-12, 1979, Kolm, W. Germany, NLR MP 79029 U

26. Davis, R.T. and Werle, M.J., Progress on Interacting Boundary-Layer Computations at High Reynolds Number, Numerical and Physical Aspects of Aerodynamic Flows, Springer-Verlag, New York, Chapter 12, pp. 187-210, 1982.

27. Werle, M.J. and Verdon, J.V., Viscid/Inviscid Interaction Analysis for Symmetric Trailing Edges, United Technologies Research Center Report, R79-914493-5, prepared under Contract N00019-78-C-0604 for the Naval Air Systems Command, January 1980.

28. Schlichting, H., Boundary Layer Theory, McGraw-Hill, Inc., New York, 1955.

29. Van Dyke, M.D., Higher Approximations in Boundary-Layer Theory, Part 1, General Analysis, J. Fluid Mech., 14, pp. 161-177, 1962.

30. Ashley, H. and Landahl, M., Aerodynamics of Wings and Bodies, Addison-Wesley, Reading,Massachusetts, 1965.

31. Reyhner, T.A. and Flügge-Lotz, I., The Interaction of a Shock Wave with a Laminar Boundary Layer, Int. J. Non-Linear Mech., Vol. 8, No. 2, pp. 173-193, June, 1968.

32. Abbott, I.H. and von Doenhoff, A.E., Theory of Wing Sections, Dover Publ., Inc., New York, 1959.

33. Bauer, F., Garabedian, P., Korn, D. and Jameson, A., Supercritical Wing Sections II, Lecture Notes in Economics and Mathematical Systems, 108, Springer-Verlag, Berlin, 1975.

34. Carter, J.E. and Wornom, S.F., Solutions for Incompressible Separated Boundary Layers Including Viscous-Inviscid Interaction, NASA SP-347, presented at NASA Conf. on Aerodynamic Analyses Requiring Advanced Computers, March 4-6, 1975.

35. Jobe, C.E. and Burggraf, O.R., The Numerical Solution of the Asymptotic Equations of Trailing Edge 
Flow, Proc. Roy. Soc. Lond. A. 340, pp. 91-111, 1974.

36. Roshko, A., On the Drag and Shedding Frequency of Two-Dimensional Bluff Bodies, NACA TN 3169, July 1954.

37. Cebeci, T. and Smith, A.M.O., Analysis of Turbulent Boundary Layers, Academic Press, New York, 1974.

38. Edwards, D.E., Carter, J.E. and Hafez, M.M., Viscous/Inviscid Interaction Analysis of Transonic Shock Induced Separated Flow Including Normal Pressure Gradients, AIAA paper 85-0371, presented at the AIAA 23rd Aerospace Sciences Meeting, January 14-17, 1985, Reno, Nevada.

39. Chang, K.C., Bui, M.N., Cebeci, T. and Whitelaw, J.H., The Calculation of Turbulent Wakes, AIAA J., Vol. 24, No. 2, pp. 200-201, February, 1986, also, Rept. ME-84-3, Heat Transfer and Fluid Mechanics Group, Department of Mechanical Engineering, California State University, Long Beach, California, September, 1984. 


\section{APPENDIX A: SOLUTION OF THE TRIPLE-DECK PROBLEM}

In order to solve the triple-deck problem, the solution to the equation

$$
\int_{0}^{x_{o}} \frac{f^{\prime}(s)}{\left(x_{o}-s\right)^{3 / 2}} d s=\frac{\alpha \pi}{x_{o}^{9 / 16} \tilde{h}}
$$

is required for $f(x)$ of the form

$$
f(x)=a_{0} x^{1 / 2}+a_{1} x+a_{2} x^{2}+a_{3} x^{3}+a_{4} x^{4}
$$

The trailing edge is located at $x=1$. Because of the singularity in the integrand at $x_{0}$, and the infinite slope at the leading edge, the integral cannot be directly evaluated for the given function, $f(x)$. To avoid the difficulties associated with the endpoints, the integral from zero to $x_{o}$ is broken into two separate integrals. Let $I$ be equal to the right-hand side of (A.1) and defne $I=I_{a}+I_{b}$ where $I_{a}$ is iniegrated fromin 0 to $x_{1}$ $\left(0<x_{1}<x_{o}\right)$ and $I_{b}$ spans the remaining interval from $x_{1}$ to $x_{0}\left(x_{0}<1\right)$. Then

$$
I_{a}=\int_{0}^{x_{1}} \frac{f^{\prime}(s)}{\left(x_{0}-s\right)^{3 / 2}} d s
$$

and the singularity at $x_{o}$ is analytically removed from $I_{b}$ by adding and subtracting $f^{\prime}\left(x_{o}\right)$ from the numerator of the integrand; the result is integrated by parts twice to yield

$$
I_{b}=-2 f^{\prime}\left(x_{1}\right)\left(x_{o}-x_{1}\right)^{-1 / 2}-4\left[f^{\prime \prime}\left(x_{1}\right)\left(x_{o}-x_{1}\right)^{1 / 2}+\int_{x_{1}}^{x_{o}} f^{\prime \prime \prime}(s)\left(x_{o}-s\right) d s\right]
$$

After substitution of Eq. (A.2) and integration, $I_{a}$ is given by

$$
\begin{gathered}
I_{a}=\left(x_{o}-x_{1}\right)^{-1 / 2}\left[a_{0} \frac{x_{1}^{1 / 2}}{x_{o}}+2 a_{1}\left\{1-\left(1-\frac{x_{1}}{x_{o}}\right)^{1 / 2}\right\}+4 a_{2}\left(x_{1}+2\left(x_{o}-x_{1}\right)^{1 / 2}\left\{\left(x_{o}-x_{1}\right)^{1 / 2}-x_{o}^{1 / 2}\right\}\right)\right. \\
+6 a_{3}\left\{x_{1}^{2}+\frac{4}{3}\left(x_{o}-x_{1}\right)^{1 / 2}\left\{\left(2 x_{o}+x_{1}\right)\left(x_{o}-x_{1}\right)^{1 / 2}-2 x_{o}^{3 / 2}\right\}\right\} \\
\left.+8 a_{4}\left\{x_{1}^{3}+\frac{2}{5}\left(x_{o}-x_{1}\right)^{1 / 2}\left(\left[8 x_{o}^{2}+4 x_{o} x_{1}+3 x_{1}^{2}\right]\left(x_{o}-x_{1}\right)^{1 / 2}-8 x_{o}^{5 / 2}\right)\right\}\right]
\end{gathered}
$$

and the integral term in Eq. (A.3b) results in

$$
\int_{x_{1}}^{x_{0}} f^{\prime \prime \prime}(s)\left(x_{o}-s\right)^{1 / 2} d s=\left(x_{o}-x_{1}\right)^{3 / 2}\left[\frac{a_{0}}{4} \frac{2 x_{1}+x_{o}}{x_{o}^{2} x_{1}^{3 / 2}\left(x_{o}-x_{1}\right)}+4 a_{3}+\frac{16}{5} a_{4}\left(2 x_{o}+3 x_{1}\right)\right]
$$

The remaining terms in $I_{b}$ are evaluated using the analytical expressions for the derivatives of $f(x)$.

An inverse procedure is employed to evaluate (A.1); i.e., instead of solving for $x_{o}$ given $\widetilde{h}, x_{o}$ is specified and $\tilde{h}$ is determined. In addition to being much more straightforward to solve as an inverse problem, $\widetilde{h}$ is a unique function of $x_{0}$, whereas the converse is not necessarily true.

The quantity $x_{1}$ can be chosen arbitrarily, but must be less than $x_{o}$, and $x_{1}$ can be placed very close to the nose, generally without difficulty. The only problem associated with the placement of $x_{1}$ is due to the possible introduction of roundoff error as $x_{1} \rightarrow 0$, however in this study values of $x_{1}$ as small as 0.001 were used without any difficulty.

Once $\widetilde{h}$ is determined, the value of $b$, the coefficient of the parabolic term in Eq. (10) describing the asymptotic downstream growth of the free streamline, must be evaluated. The value of $b$ is determined by analytically integrating Eq. (10), which is restated here for convenience, 


$$
b=\frac{2 \tilde{h}}{\pi} \int_{0}^{x_{0}} \frac{f^{\prime}(s)}{\left(x_{0}-s\right)^{1 / 2}} d s
$$

to yield

$$
b=\frac{2 \tilde{h}}{\pi}\left[\frac{a_{0}}{2} \pi+2 a_{1} \sqrt{x_{o}}+\frac{8}{3} a_{2} x_{o}^{3 / 2}+\frac{16}{5} a_{3} x_{o}^{5 / 2}+\frac{128}{35} a_{4} x_{o}^{7 / 2}\right]
$$

Recall that $b=0$ corresponds to wake cut-off; Newton's method applied to (A.6) with b equal to zero yields the cut-off value of $x_{o}$. Also, note that for values of $x_{o}$ which result in $b<0$, this analysis is not applicable; since it does not apply after the eddy has collapsed, i.e., below cut-off. 


\section{APPENDIX B: TURBULENCE MODEL}

The turbulence model used in the present study is an algebraic model which is based upon the eddyviscosity concept. Turbulence must be modeled for a boundary layer on a solid surface and for a symmetric wake. The turbulence model applied within the surface boundary layer is based upon that developed by Cebeci and Smith (Ref. 37). The reader is referred to Ref. 37 for details of the turbulence model; in this appendix the eddy-viscosity coefficient is given in its final form obtained after applying the boundary-layer transformation to Görtler variables.

In terms of the present nondimensionalization, the total viscosity, which is the sum of the molecular plus the eddy viscosity, is given (for an incompressible flow) by

$$
\mu_{t}=1+\epsilon
$$

In the Cebeci-Smith model the boundary layer is viewed as consisting of two regions, an inner and an outer region. In the inner region, which is adjacent to the body surface, the eddy viscosity coefficient $\epsilon_{i}$ is given by

$$
\epsilon_{i}=\sqrt{2 \xi R e}(\kappa \eta)^{2}[1-\exp (-y / A)]^{2}\left|\frac{\partial F}{\partial \eta}\right|
$$

where

$$
\begin{gathered}
y / A=(2 \xi R e)^{1 / 4} \tilde{N} \frac{\eta}{A^{+}}\left(\frac{\partial F}{\partial \eta}\right)_{\max }^{1 / 2} \\
\tilde{N}=\left(1-11.8 p^{+}\right)^{1 / 2}
\end{gathered}
$$

and

$$
p^{+}=(2 \xi R e)^{-1 / 4} \beta_{1}(\xi)\left(\frac{\partial F}{\partial \eta}\right)_{\max }^{-3 / 2}
$$

The subscript "max" denotes that the quantity used is the maximum calculated value occurring across the profile at the given $\xi$-location. The quantity $\kappa$ is the von Karman constant, equal to 0.40 in the present investigation, and $A^{+}$is the van Driest damping coefficient, equal here to the usual value of 26 . The only difference between this definition of the inner eddy-viscosity coefficient and that given by Cebeci and Smith (Ref. 37) is the present use of the maximum value of $\partial F / \partial \eta$ instead of the wall value used in Ref. 37 . This is done in order to avoid possible difficulties in the turbulence model near separation and reattachment points caused by the vanishingly small skin friction there. This modification to the model of Cebeci and Smith was introduced by Carter and Wornom (Ref. 34).

The eddy-viscosity coefficient in the outer region is given by

$$
\epsilon_{o}=\frac{9}{7} \sqrt{R e} U_{e} \alpha \theta
$$

where $\alpha$ is the Clauser constant, taken here to be the usual value of 0.0168 , and $\theta$ is the momentum thickness, defined by Eq. (B.4). This modification to the usual definition of the outer eddy-viscosity coefficient of Cebeci and Smith (Ref. 37), which uses the displacement thickness in place of the momentum thickness, was introduced by Edwards, et al. (Ref. 38) based upon observations of experimentally measured data in a turbulent separated flow. The $9 / 7$ factor is present so that the usual value of the outer-eddy viscosity coefficient is recovered when the flow reduces to a zero pressure gradient (flat-plate) flow.

The inner eddy-viscosity coefficient is applied from the body surface until the the smallest $\eta$-location at which $\epsilon_{i}$ exceeds $\epsilon_{o}$; above this location $\epsilon_{o}$ is applied.

The turbulence model which is adopted for the wake flow is that presented by Chang, et al. (Ref. 39) where the eddy-viscosity coefficient at a given by the expression

$$
\epsilon(\eta)=\epsilon_{w}+\left\{\epsilon_{T E}(\eta)-\epsilon_{w}\right\} \exp \left[\sqrt{\operatorname{Re}}\left(x_{T} E-x\right) /\left(20 \delta_{T E}^{*}\right)\right]
$$


where

$$
\epsilon_{w}=0.064 \sqrt{R e} \frac{9}{7} \theta U_{e}
$$

The subscripts " $T E$ " and " $w$ " denote a quantities evaluated at the trailing-edge station and along the wake, respectively, and $\theta_{W}$ is the momentum thickness across half of the symmetric wake, where the momentum thickness is defined by the expression

$$
\theta=\frac{\sqrt{2 \xi}}{U_{e}} \int_{0}^{\infty} F\left(1-\frac{U_{e_{o}}}{U_{e}} F\right) d \eta
$$

The momentum thickness has been substituted for the displacement thickness used in the original model presented in Ref. 38 for consistency with the turbulence model applied along the airfoil surface. 


\section{APPENDIX C: BOUNDARY-LAYER EQUATION COEFFICIENTS}

The coefficients of the finite-difference form of the continuity and momentum equations are defined below. For the continuity equation, (39), the coefficients are given at $i, j-1 / 2$ (see Fig. 4) by

$$
P_{j}=\Delta \eta^{-}\left(\frac{1}{2}+\frac{\xi_{i}}{\Delta \xi}\right)
$$

and

$$
Q_{j}=\xi_{i} \frac{\Delta \eta^{-}}{\Delta \xi}\left(F_{i-1, j}+F_{i-1, j-1}\right)
$$

For the momentum equation, (45), the coefficients at $i, j$ are given by

$$
\begin{gathered}
\bar{A}_{j}=\frac{2 \bar{\epsilon}^{-}}{\Delta \eta^{-}\left(\Delta \eta^{+}+\Delta \eta^{-}\right)}+(1-\tilde{\theta})\left(\frac{\bar{V}^{*}}{\Delta \eta^{-}}+\frac{\omega}{2} V_{\eta}^{*}\right)_{j-1 / 2}+\left(\omega-\beta_{o_{i}}\right)(1-\tilde{\theta}) F_{i, j-1}^{*} \\
\bar{B}_{j}=\frac{-2}{\Delta \eta^{+}+\Delta \eta^{-}}\left(\frac{\bar{\epsilon}^{+}}{\Delta \eta^{+}}+\frac{\bar{\epsilon}^{-}}{\Delta \eta^{-}}\right)+\tilde{\theta}\left(\frac{\bar{V}^{*}}{\Delta \eta^{+}}+\frac{\omega}{2} V_{\eta}^{*}\right)_{j+1 / 2} \\
+(1-\tilde{\theta})\left(-\frac{\bar{V}^{*}}{\Delta \eta^{-}}+\frac{\omega}{2} V_{\eta}^{*}\right)_{j-1 / 2}+\left(\omega-\beta_{o_{i}}\right) F_{i, j}^{*} \\
\bar{C}_{j}=\frac{2 \bar{\epsilon}^{+}}{\Delta \eta^{+}\left(\Delta \eta^{+}+\Delta \eta^{-}\right)}+\tilde{\theta}\left(-\frac{\bar{V}^{*}}{\Delta \eta^{+}}+\frac{\omega}{2} V_{\eta}^{*}\right)_{j+1 / 2}+\left(\omega-\beta_{o_{i}}\right) \tilde{\theta} F_{i, j+1}^{*} \\
\bar{D}_{j}=-(1-\tilde{\theta})\left(F_{\eta}^{*} / 2+\omega \bar{F}^{*} / \Delta \eta^{-}\right)_{j-1 / 2} \\
\bar{E}_{j}=-\tilde{\theta}\left(F_{\eta}^{*} / 2+\omega \bar{F}^{*} / \Delta \eta^{+}\right)_{j+1 / 2}-(1-\tilde{\theta})\left(F_{\eta}^{*} / 2-\omega \bar{F}^{*} / \Delta \eta^{-}\right)_{j-1 / 2} \\
\bar{F}_{j}=-\tilde{\theta}\left(F_{\eta}^{*} / 2-w \bar{F}^{*} / \Delta \eta^{+}\right)_{j+1 / 2} \\
\bar{G}_{j}=1
\end{gathered}
$$

and

$$
\begin{gathered}
\bar{H}_{j}=\frac{\omega-\beta_{o_{i}}}{2}\left[\widetilde{\theta} F_{i, j+1}^{* 2}+F_{i, j}^{* 2}+(1-\tilde{\theta}) F_{i, j-1}^{* 2}\right] \\
+\widetilde{\theta}\left[-\bar{V}^{*} F_{\eta}^{*}+\omega \bar{F}^{*} V_{\eta}^{*}\right]_{j+1 / 2}+(1-\tilde{\theta})\left[-\bar{V}^{*} F_{\eta}^{*}+\omega \bar{F}^{*} V_{\eta}^{*}\right]_{j-1 / 2}
\end{gathered}
$$

where the asterisk denotes a "guessed" value of the quantity, usually taken from the previous global iteration at the same $i, j$ location, the overbar denotes an average value given by the general notation for a quantity $q$,

$$
\bar{q}_{j \pm 1 / 2}=\frac{1}{2}\left(q_{j}+q_{j \pm 1}\right)
$$

and the $\eta$ derivatives are defined by

$$
q_{\eta_{j \pm 1 / 2}}=\mp\left(q_{j}-q_{j \pm 1}\right) / \Delta \eta^{ \pm}
$$

The quantity $\omega$ is the FLARE coefficient (Ref. 31) which, if FLARE is employed, is set to zero when $F_{i, j}^{*}<0$, otherwise $\omega$ is equal to one. The quantity $\tilde{\theta}$ is the weighting function for nonuniform $\eta$-grids, defined by Eq. (43). 


\section{APPENDIX D: BOUNDARY-LAYER EQUATION SOLUTION}

The boundary-layer equations written in finite-difference form constitute a linear system of coupled algebraic equations which can be written as a block tridiagonal matrix where each block is $2 \times 2$. An efficient inversion algorithm can be developed based upon an extension of the Thomas algorithm for a single equation. The inversion scheme is developed by assuming that the solution for the dependent variables $F$ and $V$ can be expressed as recursion relations in the form

$$
F_{i, j}=Z_{1 j}+P_{1 j} F_{i, j+1}+Q_{1_{j}} V_{i, j+1}+R_{1_{j}} \beta_{1_{i}}
$$

and

$$
V_{i, j}=Z_{2 j}+P_{2 j} F_{i, j+1}+Q_{2 j} V_{i, j+1}+R_{2 j} \beta_{1_{i}}
$$

By substituting from the recursion relations for $F_{i, j-1}$ and $V_{i, j-1}$ into the continuity and momentum equations those equations can be written in the form

$$
S_{1} F_{i, j}+S_{2} V_{i, j}+S_{3} \beta_{1 i}=S_{4}
$$

and

$$
T_{1} F_{i, j}+T_{2} V_{i, j}+T_{3} \beta_{1 i}=T_{4}-\bar{C}_{j} F_{i, j+1}-\bar{F}_{j} V_{i, j+1}
$$

respectively, where

$$
\begin{gathered}
S_{1}=P_{2_{j-1}}-P_{j}\left(1+P_{1_{j-1}}\right) \\
S_{2}=Q_{2_{j-1}}-1-P_{j} Q_{j-1} \\
S_{3}=R_{2_{j-1}}-P_{j} R_{1_{j-1}} \\
S_{4}=P_{j} Z_{1_{j-1}}-Q_{j}-Z_{2_{j-1}} \\
T_{1}=\bar{A}_{j} P_{1_{j-1}}+\bar{B}_{j}+\bar{D}_{j} P_{2_{j-1}} \\
T_{2}=\bar{A}_{j} Q_{1_{j-1}}+\bar{E}_{j}+\bar{D}_{j} Q_{2_{j-1}} \\
T_{3}=\bar{A}_{j} R_{1_{j-1}}+\bar{G}_{j}+\bar{D}_{j} R_{2_{j-1}}
\end{gathered}
$$

and

$$
T_{4}=\bar{H}_{j}-\bar{A}_{j} Z_{1_{j-1}}-\bar{D}_{j} Z_{2_{j-1}}
$$

Equations (D.2a) and (D.2b) can be solved simultaneously for $F_{i, j}$ and $V_{i, j}$ to give the recursion relation coefficients:

$$
\begin{gathered}
Z_{1 j}=U_{1}\left(T_{2} S_{4}-T_{4} S_{2}\right) \\
P_{1 j}=U_{1} S_{2} \bar{C}_{j} \\
Q_{1 j}=U_{1} S_{2} \bar{F}_{j}
\end{gathered}
$$




$$
\begin{gathered}
R_{1 j}=U_{1}\left(T_{3} S_{2}-T_{2} S_{3}\right) \\
Z_{2 j}=U_{1}\left(S_{1} T_{4}-S_{4} T_{1}\right) \\
P_{2 j}=-U_{1} S_{1} \bar{C}_{j} \\
Q_{2 j}=-U_{1} S_{1} \bar{F}_{j}
\end{gathered}
$$

and

$$
R_{2 j}=U_{1}\left(T_{1} S_{3}-T_{3} S_{1}\right)
$$

where

$$
U_{1}=1 /\left(S_{1} T_{2}-S_{2} T_{1}\right)
$$

In order to determine the recursion relation coefficients, they are initialized at the surface/wakecenterline boundary using the appropriate physical boundary conditions. On the body surface the slip and specified injection boundary conditions are applied, i.e., $F_{i, 1}=F_{w}\left(\xi_{i}\right), V_{i, 1}=V_{w}\left(\xi_{i}\right)$, so that at $j=1$ all of the coefficients in Eqs. (D.1) are zero except for $Z_{1}$ and $Z_{2}$, which are equal to $F_{w}$ and $V_{w}$, respectively. If the lower boundary is located at a symmetric wake centerline, then $V_{i, 1}=0$, so that the four coefficients in (D.1b) are zero at $j=1$. The wake centerline condition is written in the form of Eq. (47b), where the coefficients in that equation are defined by

$$
\begin{gathered}
A_{o}=-\frac{2 \bar{\epsilon}_{w}}{\Delta \eta^{2}}+\left(\omega-\beta_{o_{i}}\right)\left(F_{i, 1}^{*}+F_{i, 2}^{*} / 2\right)+\omega V_{i, 2}^{*} / \Delta \eta \\
B_{o}=\frac{2 \bar{\epsilon}_{w}}{\Delta \eta^{2}}+\frac{\left(\omega-\beta_{o_{i}}\right)}{2} F_{i, 1}^{*} \\
C_{o}=\frac{\omega}{\Delta \eta} F_{i, 1}^{*} \\
D_{o}=1
\end{gathered}
$$

and

$$
E_{o}=F_{i, 1}^{*}\left[\frac{\omega}{\Delta \eta} V_{i, 2}^{*}+\frac{\left(\omega-\beta_{o_{i}}\right)}{2}\left(F_{i, 1}^{*}+F_{i, 2}^{*}\right)\right]
$$

This equation is of the form of Eq. (D.1a), so that the recursion relation coefficients at $j=1$ are on a symmetric wake centerline by

$$
\begin{gathered}
Z_{1_{1}}=E_{o} / A_{o} \\
P_{1_{1}}=-B_{o} / A_{o} \\
Q_{1_{1}}=-C_{0} / A_{o}
\end{gathered}
$$

and

$$
R_{1_{1}}=-D_{o} / A_{o}
$$


With the above expressions for the recursion relation coefficients at the lower boundary $(j=1)$, the remaining coefficients can be determined across the entire boundary layer. The solution for the dependent flow variables cannot be completed until the viscous outer edge condition is imposed at $j=N J$ in conjunction with the coupling of the viscous and inviscid flow regions. This condition is given by Eq. (48) which is rewritten, after substitution from the governing inviscid law for the edge velocity, either Eq. (52) or (57) expressed in the form (56c), as

$$
W F_{i, N J}+X V_{i, N J}+Y V_{i, N J-1}=S
$$

where

$$
\begin{gathered}
W=-\frac{\sqrt{2 \xi_{i}}}{\Delta \xi} \frac{U_{e_{o} i}}{\widetilde{D}_{i}} \\
X=1-\eta_{N J} / \Delta \eta \\
Y=\eta_{N J} / \Delta \eta
\end{gathered}
$$

and

$$
S=-\frac{\sqrt{2 \xi_{i}}}{\Delta \xi}\left[\frac{\widetilde{C}_{i}}{\widetilde{D}_{i}}+\left(U_{e} \delta^{*}\right)_{i-1}\right]+V_{w}\left(\xi_{i}\right)
$$

The quantity $U_{e_{i}}$ in Eq. (56c) has been eliminated in favor of $F_{i, N J}$ using the edge relation for $F$, Eq. (49), and the resulting expression has then been used to eliminate $U_{e_{i}}$ in (48). In the above relations $\Delta \eta=\eta_{N J}-\eta_{N J-1}$.

The final relation needed to solve for the unknowns at the edge of the boundary layer is obtained by writing the momentum equation there noting that the edge condition (27) is attained exponentially, so that all of the derivatives of $F$ go to zero at the edge of the boundary layer. The momentum equation can be written in the form of Eq. (50) where the coefficients are defined by

$$
\begin{gathered}
\bar{W}=\left(V_{i, N J}^{*}-V_{i, N J-1}^{*}\right) / \Delta \eta+2\left(1-\beta_{o_{i}}\right) F_{i, N J}^{*} \\
\bar{X}=F_{i, N J}^{*} / \Delta \eta \\
\bar{Y}=-F_{i, N J}^{*} / \Delta \eta
\end{gathered}
$$

and

$$
\bar{S}=F_{i, N J}^{*}\left(V_{i, N J}^{*}-V_{i, N J-1}^{*}\right) / \Delta \eta+\left(1-\beta_{o_{i}}\right) F_{i, N J}^{* 2}
$$

Using the edge boundary condition on $V$ (Eq. D.6a), the momentum equation (50), the two recursion relations, (D.1a) and (D.1b), and the continuity equation (39) written at $N J-1 / 2$, a set of five equations in 5 unknowns results at the edge of the boundary layer. This set is solved yielding $F$ and $V$ at $i, N J$ and $i, N J-1$ and $\beta_{1_{i}}$. Having $F_{i, N J}$ in turn yields $U_{e_{i}}$ and $\left(U_{e} \delta^{*}\right)_{i}$. With the edge values of the dependent variables $F$ and $V$ known, the remaining unknown variables are obtained by sweeping the recursion relations from $j=N J$ to $j=1$. 


\section{APPENDIX E: INVISCID INTEGRAL REPRESENTATION COEFFICIENTS}

The coefficients appearing in the discrete forms of the inviscid integrals are given in this appendix. In addition, the inviscid integral spanning the region downstream of the strong-interaction region is evaluated for specified analytical behavior of the displacement thickness distribution there.

In the present investigation only blunt-nosed bodies are considered, for which the upstream behavior of the displacement thickness distribution cannot be specified in a known analytical form. Therefore the strong-interaction region begins at the leading edge where a stagnation point similarity solution is assumed to give the correct boundary-layer starting profile. All of the geometries considered in this study are finite bodies, and the local strong-interaction calculations are performed with the strong-interaction region ending in the wake. In the massive separation calculations, the downstream region is completely accounted for within the inviscid integral spanning $x=0$ to $x=x_{o}$, so that there is no downstream integral, in contrast with the local strong-interaction procedure.

Because all of the bodies analyzed here have blunt noses, the parabola baseline coordinate is utilized. Therefore all of the results presented in this appendix are developed assuming that the baseline coordinate is a parabola. Note that as a consequence the Görtler variable $\xi$ appears in place of $x$; the latter would be used if the $x$-axis were the baseline coordinate curve. Note, however, that for a parabola baseline $\xi$ is equal to the Cartesian $x$ value corresponding to the given location on the baseline surface.

In order to recover the results for the $x$-axis baseline coordinate, in the relations presented below replace $\xi$ by the Cartesian $x$, set $U_{e_{0}}$ equal to one and, in the local-strong interaction calculations $f$ is the distance from the $x$-axis to the body surface, therefore $f$ is zero in the wake.

It is assumed in the local strong-interaction calculations that the downstream end of the stronginteraction calculation domain is aft of the trailing edge.

\section{Coefficients of Compact Discretized Inviscid Equation}

The general compact discrete form for the inviscid integrals given by Eq. (57c) is rewritten, for the case where a baseline coordinate is employed which does not coincide with the $x$-axis, in the form

$$
F_{i, N J}=U_{e_{i}} / U_{e_{o i}}=\widetilde{C}_{i}+\widetilde{D}_{i}\left(U_{e} \delta_{i}^{*}\right)
$$

The coefficients $\widetilde{C}$ and $\widetilde{D}$ appearing in the above equation are defined below.

For the local strong-interaction calculations $\widetilde{C}$ and $\widetilde{D}$ are determined from (57b) to be

$$
\widetilde{C}_{i}=\bar{g}_{i}+\frac{1}{\pi} \sum_{\substack{j=1 \\ j \neq i}}^{I E} T_{j} D_{i j}+\frac{D_{i i}}{\pi \sqrt{R e}}\left(U_{e_{o}} f\right)_{i}
$$

and

$$
\widetilde{D}_{i}=\frac{D_{i i}}{\pi \sqrt{R e}}
$$

In (E.2) the term $\bar{g}_{i}$ is obtained from Eq. (56) with $\xi_{I}=0$ :

$$
\bar{g}_{i}=1+\frac{1}{\pi}\left[\frac{T\left(\xi_{F}\right)}{\xi_{i}-\xi_{F}}+\int_{\xi_{F}}^{\infty} \frac{T^{\prime}(t)}{\xi_{i}-t} d t\right]
$$

For the massive-separation calculations the coefficients in (E.1) are determined from (61):

$$
\tilde{C}_{i}=\bar{g}_{i}+\frac{\left(\xi_{o}-\xi_{i}\right)^{1 / 2}}{\pi}\left[\sum_{\substack{j=1 \\ j \neq i}}^{M}\left(T_{j}-P_{j}\right) D_{i j}+D_{i i}\left\{\frac{\left(U_{e_{o}} f\right)_{i}}{\sqrt{R e}}-P_{i}\right\}\right]
$$

and 


$$
\widetilde{D}_{i}=\frac{\left(\xi_{o}-\xi_{i}\right)^{1 / 2}}{\pi \sqrt{R e}} D_{i i}
$$

where

$$
P_{j}=T\left(\xi_{o}\right)+\left(\xi_{j}-\xi_{o}\right) T^{\prime}\left(\xi_{o}\right)
$$

The term $\bar{g}_{i}$ in (E.5) is defined by

$$
\bar{g}_{i}=1+\left(\frac{I}{U_{e_{o}}}\right)_{i}+\frac{T^{\prime}\left(\xi_{o}\right)}{\pi} \ln \left\{\frac{\sqrt{\xi_{o}}-\sqrt{\xi_{o}-\xi_{i}}}{\sqrt{\xi_{o}}+\sqrt{\xi_{o}-\xi_{i}}}\right\}
$$

where $\left(I / U_{e_{0}}\right)_{i}$ is defined by Eq. (36c).

\section{Downstream Contribution to Inviscid Integral}

The contribution to the inviscid integral used in the local strong-interaction procedure is evaluated below for a baseline curve which does not coincide with the $x$-axis. The boundary-layer displacement thickness in the wake downstream of a finite body is assumed to behave, when the flow is laminar, as predicted for a Goldstein wake. In the wake downstream of the body the inviscid edge velocity recovers to the freestream velocity so that $U_{e}=1+O(\epsilon)$, therefore the product of the edge velocity and the displacement thickness are assumed to behave in the same way as the displacement thickness alone, so that one can write

$$
\left(U_{e} \delta^{*}\right)(x)=0.664+C_{1} x^{-1 / 2}
$$

The unknown coefficient $C_{1}$ is determined in a manner which insures that the displacement thickness downstream of the strong-interaction region merges continuously with that upstream, which is accomplished by evaluating (E.9a) at the final boundary-layer calculation point at $i=I E$ using the known value of $U_{e} \delta^{*}$ there, which yields

$$
C_{1}=\left[\left(U_{e} \delta^{*}\right)_{I E}-0.664\right] \sqrt{x_{I E}}
$$

The ultimate goal is to evaluate the integral $I_{d}$ defined by

$$
I_{d}=\int_{\xi_{F}}^{\infty} \frac{T^{\prime}(t)}{\xi_{i}-t} d t
$$

Upon substitution of the definition of $T$, Eq. (53), with (E.9a) applied, into (E.10), evaluation of the resulting integral yields

$$
\begin{gathered}
\int_{\xi_{F}}^{\infty} \frac{T^{\prime}(t)}{\xi_{i}-t} d t=-\frac{C_{1}}{R e^{1 / 2} \xi_{i}}\left[\frac{1}{\xi_{F}^{1 / 2}}-\frac{1}{2 \xi_{i}^{1 / 2}} \ln \left\{\frac{\sqrt{\xi_{F}}+\sqrt{\xi_{i}}}{\left.\left.\sqrt{\xi_{F}-\sqrt{\xi_{i}}}\right\}\right]}\right.\right. \\
\frac{+R e^{-1 / 2} \int_{\xi_{F}}^{\infty} f^{\prime}(t)}{x i_{i}-t d t}
\end{gathered}
$$

where in the region downstream of $x_{0}$ it has been assumed that $U_{e_{o}}$ is very close to one, which is satisfied very well when the baseline parabola is thin, as is the case for most of the airfoils analyzed here. The remaining integral in (E.11) is evaluated for the parabola baseline coordinate, for which $f$ is given by

$$
f(\xi)=-\left(2 r_{o} \xi+r_{o}^{2}\right)^{1 / 2}
$$

where $r_{o}$ is the nose radius of both the airfoil and the parabola. The result of the integration is

$$
\int_{\xi_{F}}^{\infty} \frac{f^{\prime}(t)}{\xi_{i}-t} d t=\left(1+\frac{2 \xi_{i}}{r_{o}}\right)^{-1 / 2} \ln \left[\frac{\sqrt{\xi_{F} / r_{o}^{2}}+\sqrt{1+2 \xi_{i} / r_{o}}}{\sqrt{\xi_{F} / r_{o}^{2}}-\sqrt{1+2 \xi_{i} / r_{o}}}\right]
$$


Note that, since the coefficient $C_{1}$ involves $\left(U_{e} \delta^{*}\right)_{I E}$, the contribution to the term $\left(U_{e} \delta^{*}\right)_{I E}$ can be implicitly incorporated into the integral over the strong-interaction region by extracting it from (E.11) and modifying the influence coefficients $D_{i, I E}$ which multiply $\left(U_{e} \delta^{*}\right)_{I E}$ in Eq. (55a). This is done in the present analysis.

If the flow is turbulent, the displacement thickness in the wake decays like a Goldstein wake, with the displacement thickness a function of $x^{-1 / 2}$, however the constant coefficient 0.664 in Eq. (E.9a) must be determined from the upstream solution for each problem, rather than being specified. In the turbulent flow calculations performed in the present study using the local strong-interaction procedure, the above analysis to determine the downstream contribution to the Cauchy integral is repeated with (E.9a) replaced by the relation

$$
\left(U_{e} \delta^{*}\right)(x)=C_{0}+C_{1} \xi^{-1 / 2}
$$

where the coefficients $C_{0}$ and $C_{1}$ are determined by evaluating (E.14a) for $U_{e} \delta^{*}$ at $i=I E$ and $i=I E-1$ and are given by

$$
C_{0}=\left(U_{e} \delta^{*}\right)_{I E}\left[1-\left(1-\sqrt{\xi_{I E} / \xi_{I E-1}}\right)^{-1}\right]+\left(U_{e} \delta^{*}\right)_{I E-1}\left(1-\sqrt{\xi_{I E} / \xi_{I E-1}}\right)^{-1}
$$

and

$$
C_{1}=\left\{\left(U_{e} \delta^{*}\right)_{I E}-\left(U_{e} \delta^{*}\right)_{I E-1}\right\} \xi_{I E}^{1 / 2}\left(1-\sqrt{\xi_{I E} / \xi_{I E-1}}\right)^{-1}
$$

The integrations are carried out as before which yields

$$
\int_{\xi_{F}}^{\infty} \frac{T^{\prime}(t)}{\xi_{i}-t} d t=\bar{g}_{d_{1}}\left[\left(U_{e} \delta^{*}\right)_{I E}-\left(U_{e} \delta^{*}\right)_{I E-1}\right]+\bar{g}_{d_{2}}
$$

where

$$
\bar{g}_{d_{1}}=\left\{2 \xi_{i}\left(\xi_{I E}^{-1 / 2}-\xi_{I E-1}^{-1 / 2}\right) R e^{1 / 2}\right\}^{-1}\left(\frac{2}{\sqrt{\xi_{F}}}-\frac{1}{\sqrt{\xi_{i}}} \ln \left[\frac{\sqrt{\xi_{F}}+\sqrt{\xi_{i}}}{\sqrt{\xi_{F}}-\sqrt{\xi_{i}}}\right]\right)
$$

and

$$
\bar{g}_{d_{2}}=\left(1+\frac{2 \xi_{i}}{r_{o}}\right)^{-1 / 2} \ln \left[\frac{\sqrt{1+2 \xi_{F} / r_{o}}+\sqrt{1+2 \xi_{i} / r_{o}}}{\sqrt{1+2 \xi_{F} / r_{o}}-\sqrt{1+2 \xi_{i} / r_{o}}}\right]
$$

As is done for the laminar wake, the contributions to the term $U_{e} \delta^{*}$ at $i=I E$ and $i=I E-1$ are implicitly included in the solution within the strong-interaction domain by modifying the influence coefficients for those terms, $D_{i, I E}$ and $D_{i, I E-1}$, accordingly.

\section{Downstream Displacement Thickness Distribution for Massive Separation}

Once a converged massive separation solution for a particular geometry has been obtained, the displacement thickness distribution in the region downstream of $\xi_{o}$ can be obtained by integrating Eq. (36b), which is restated below for convenience, using Eq. (53):

$$
T^{\prime}(\xi)=\frac{\sqrt{\xi-\xi_{o}}}{\pi} \int_{0}^{\xi_{0}} \frac{T^{\prime}(t) d t}{\sqrt{\xi_{o}-t}(\xi-t)}+I(\xi)
$$

The term $I(\xi)$ in (E.16) is not the same when $\xi>\xi_{0}$ as that given by Eq. (36c) for $\xi<\xi_{0}$. Its form in the former case has not been determined here, because it was found that this term can be neglected in (E.16) without inducing any anomalies in the displacement thickness distribution in region immediately downstream of $\xi_{0}$. Therefore the integration is carried out below neglecting the term $I(\xi)$.

Integrating both sides of (E.16) yields 


$$
T(\xi)=T\left(\xi_{o}\right)+\frac{1}{\pi} \int_{0}^{\xi_{0}}\left\{\int_{\xi_{0}}^{\xi} \frac{\left(\tilde{\xi}-\xi_{0}\right)^{1 / 2}}{(\tilde{\xi}-\bar{\xi})} d \tilde{\xi}\right\} \frac{T^{\prime}(\bar{\xi})}{\left(\xi_{0}-\bar{\xi}\right)^{1 / 2}} d \bar{\xi}
$$

which, after a good deal of algebra gives the final result

$$
T(\xi)=T\left(\xi_{0}\right)+\frac{2}{\pi} I_{T}
$$

where

$$
\begin{aligned}
I_{T} & =\left\{T\left(\xi_{o}\right)-\xi_{o} T^{\prime}\left(\xi_{o}\right)\right\}\left\{\sqrt{\xi / \xi_{o}-1}-\tan ^{-1} \sqrt{\xi / \xi_{o}-1}\right\} \\
& +T^{\prime}\left(\xi_{o}\right)\left[2 \sqrt{\xi_{o}\left(\xi-\xi_{o}\right)}-\left(\xi-\xi_{o}\right)\left\{\frac{\xi}{\xi-\xi_{o}} \tan ^{-1} \sqrt{\xi / \xi_{o}-1}+\sqrt{\xi_{o} /\left(\xi-\xi_{o}\right)}-\frac{\pi}{2}\right\}\right] \\
& +\sum_{j=2}^{M}\left(T_{j}-P_{j}\right)\left[\tan ^{-1} \sqrt{\frac{\xi-\xi_{o}}{\xi_{o}-\bar{\xi}_{j}}}-\tan ^{-1} \sqrt{\frac{\xi-\xi_{o}}{\xi_{o}-\bar{\xi}_{j-1}}}-\sqrt{\xi-\xi_{o}}\left\{\left(\xi_{o}-\bar{\xi}_{j}\right)^{-1 / 2}-\left(\xi_{o}-\bar{\xi}_{j-1}\right)^{-1 / 2}\right\}\right]
\end{aligned}
$$

with

$$
\bar{\xi}_{j}=\left(\xi_{j}+\xi_{j+1}\right) / 2
$$

The summation appearing on the right-hand side of Eq. (E.18b) results from the integration over the stronginteraction domain, which must be done numerically, and is performed on the same grid used for the original boundary-layer calculations. 


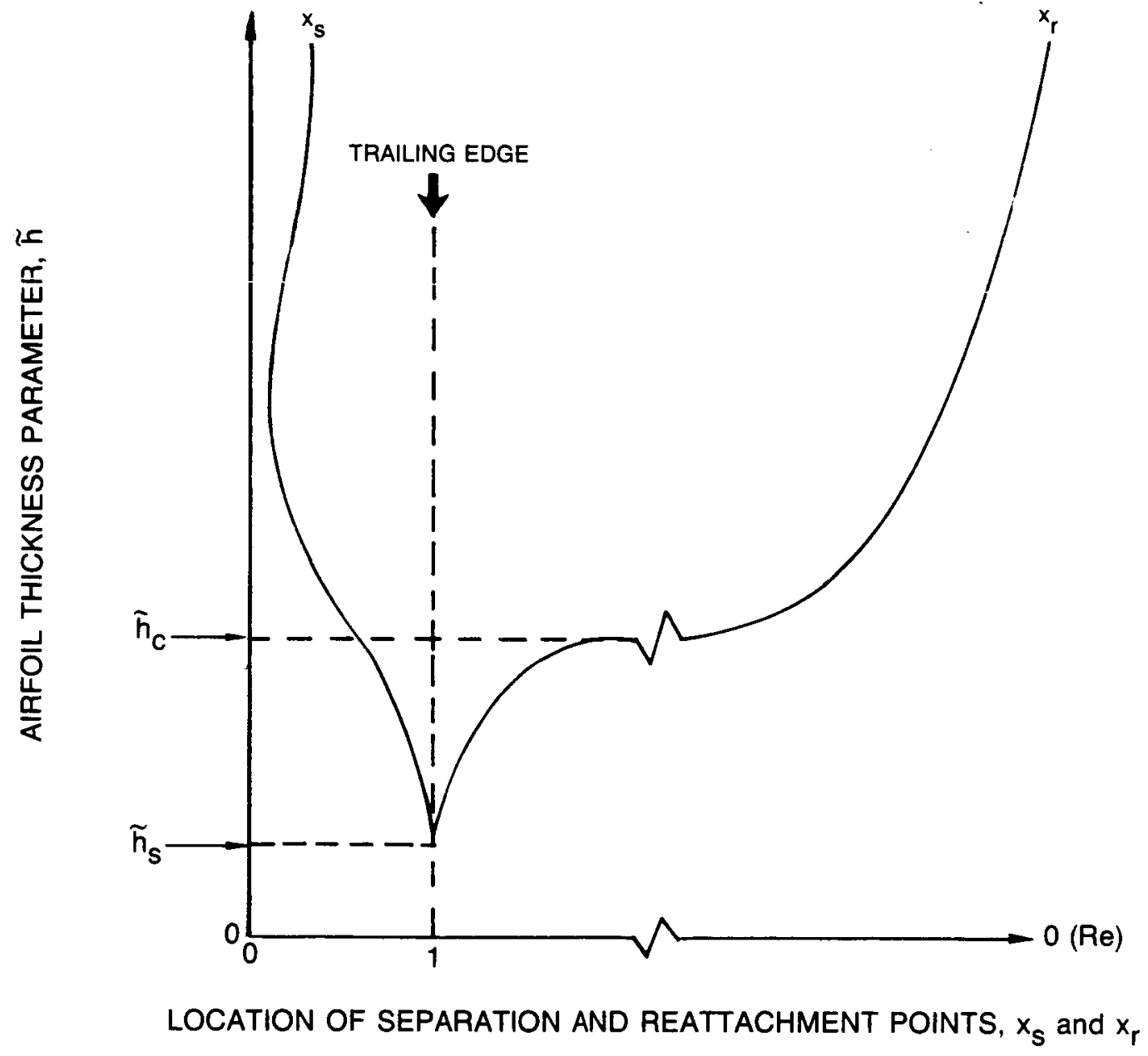

Fig. 1 Sketch of Separation and Reattachment Point Locations for Wedge-Shaped Trailing-Edge Airfoil from Triple-Deck Theory (Adapted From Ref. 17) 


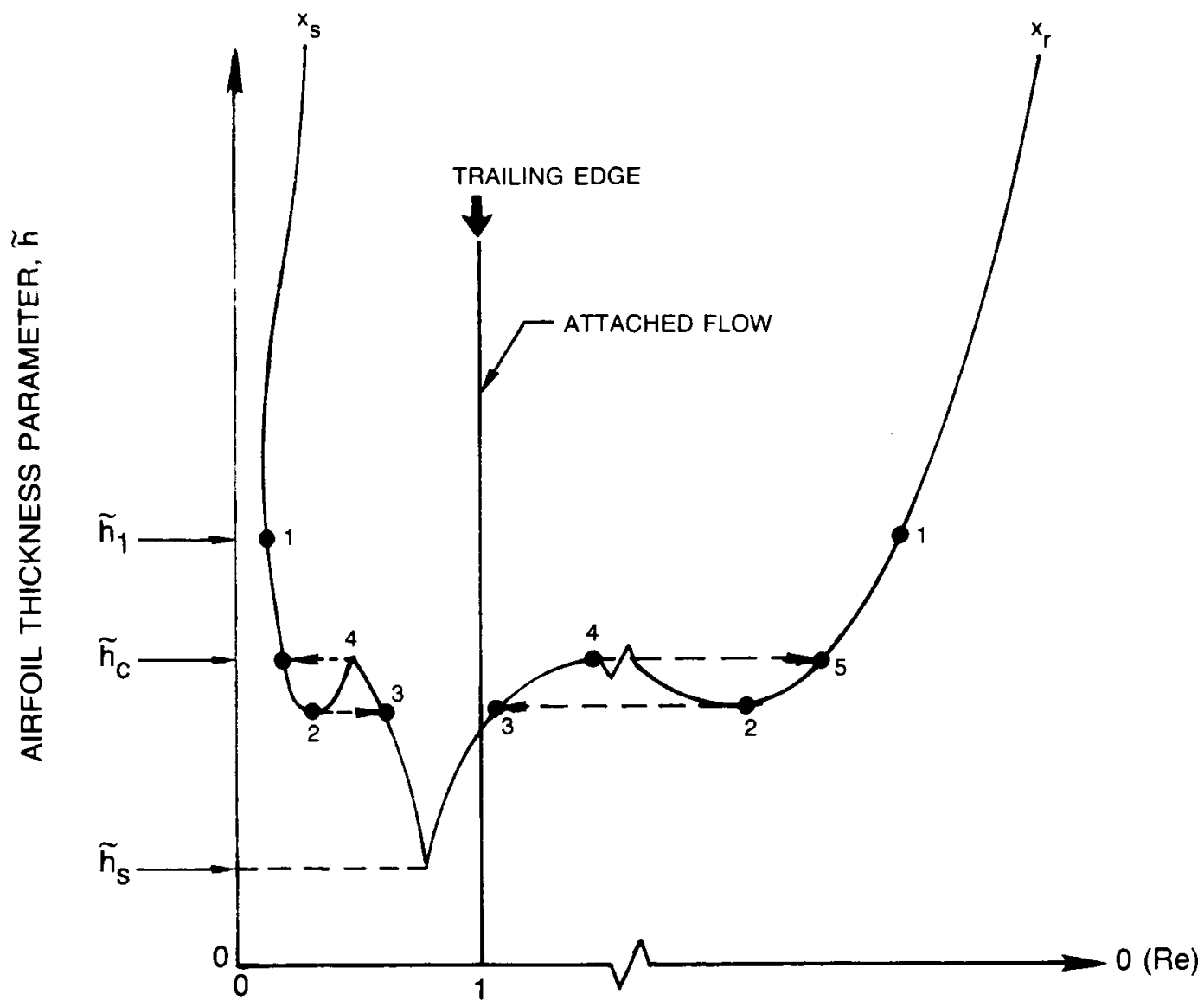

LOCATION OF SEPARATION AND REATTACHMENT POINTS, $x_{s}$ and $x_{r}$

Fig. 2 Sketch of Separation and Reattachment Point Locations for Cusped Trailing-Edge Airfoil From Triple-Deck Theory (Adapted From Ref. 17) 


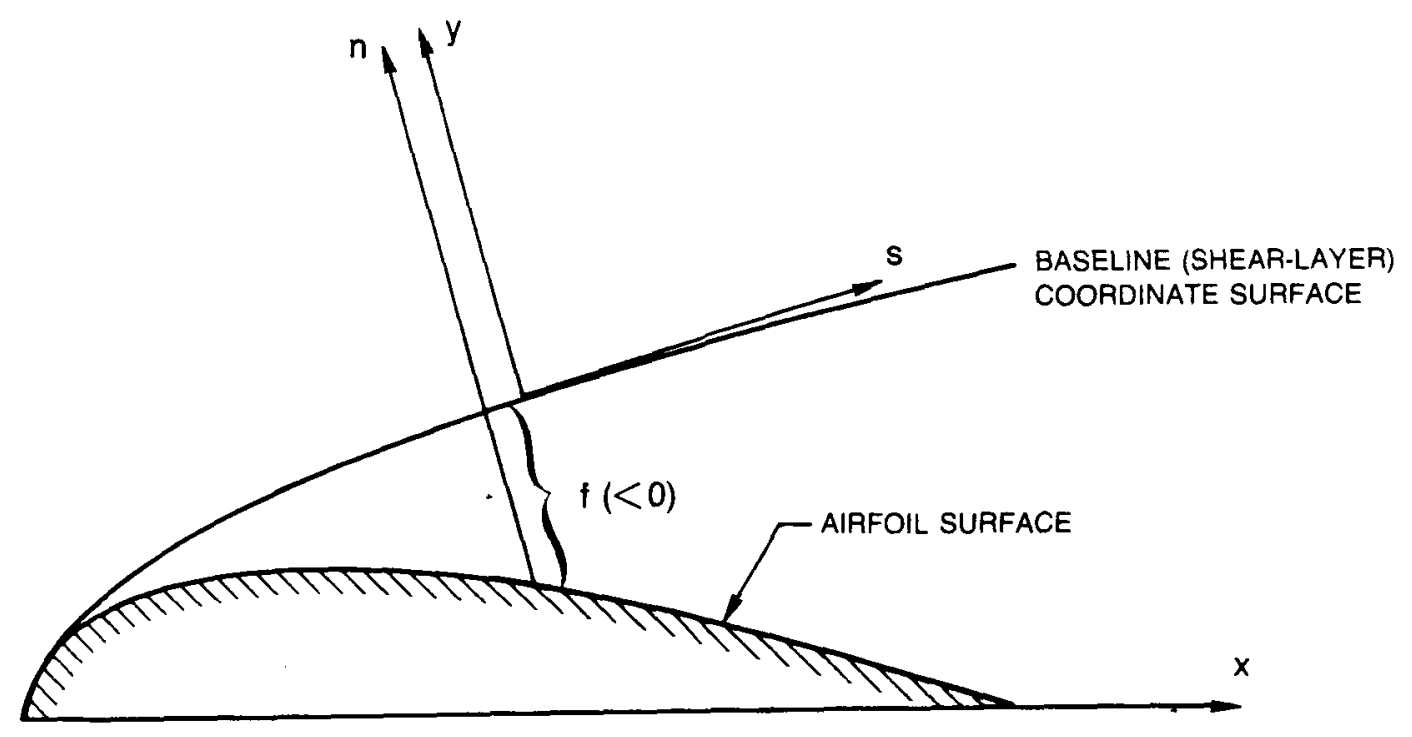

Fig. 3 Definition of Coordinate Systems 


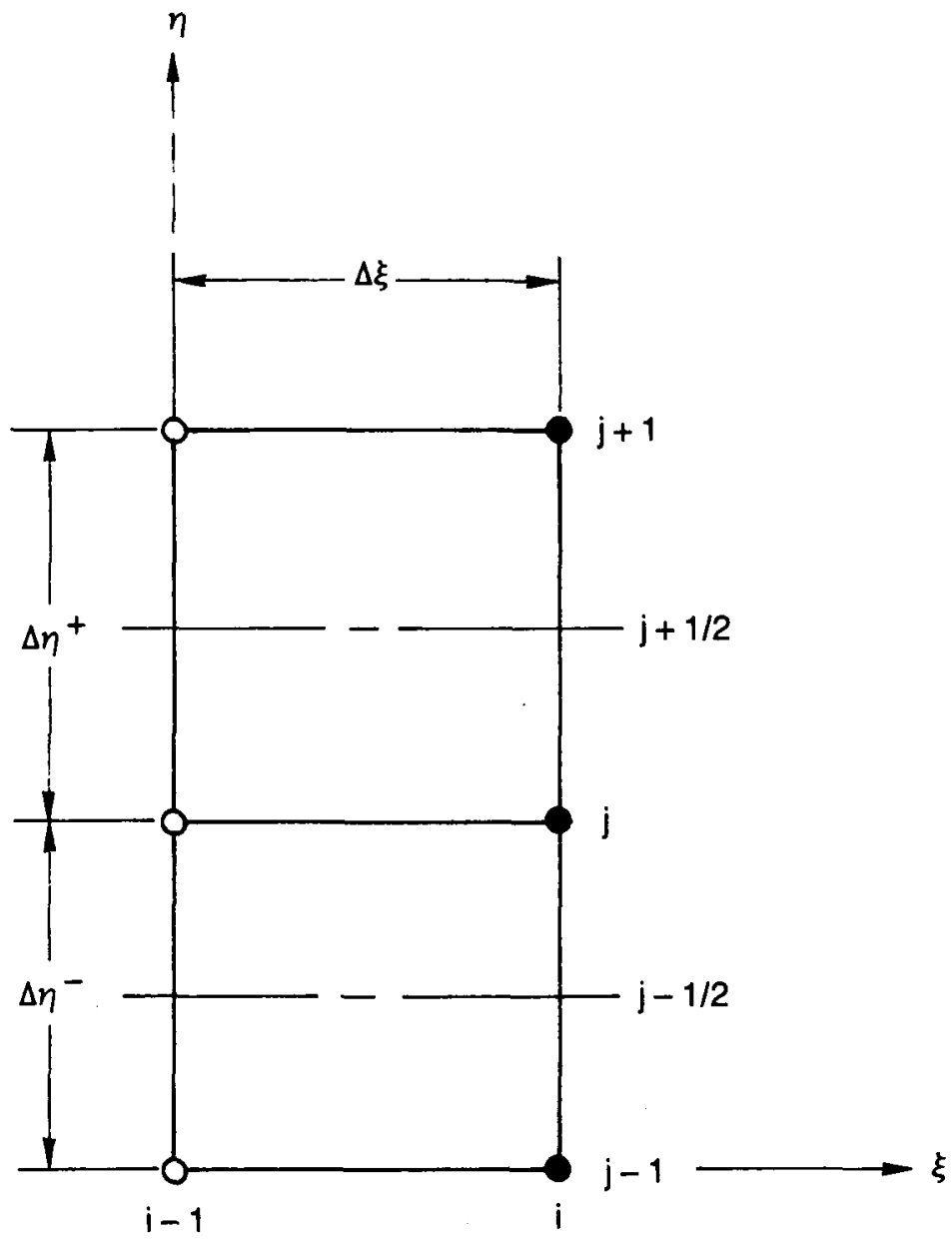

Fig. 4 Nomenclature for Boundary-Layer Finite-Difference Mesh 


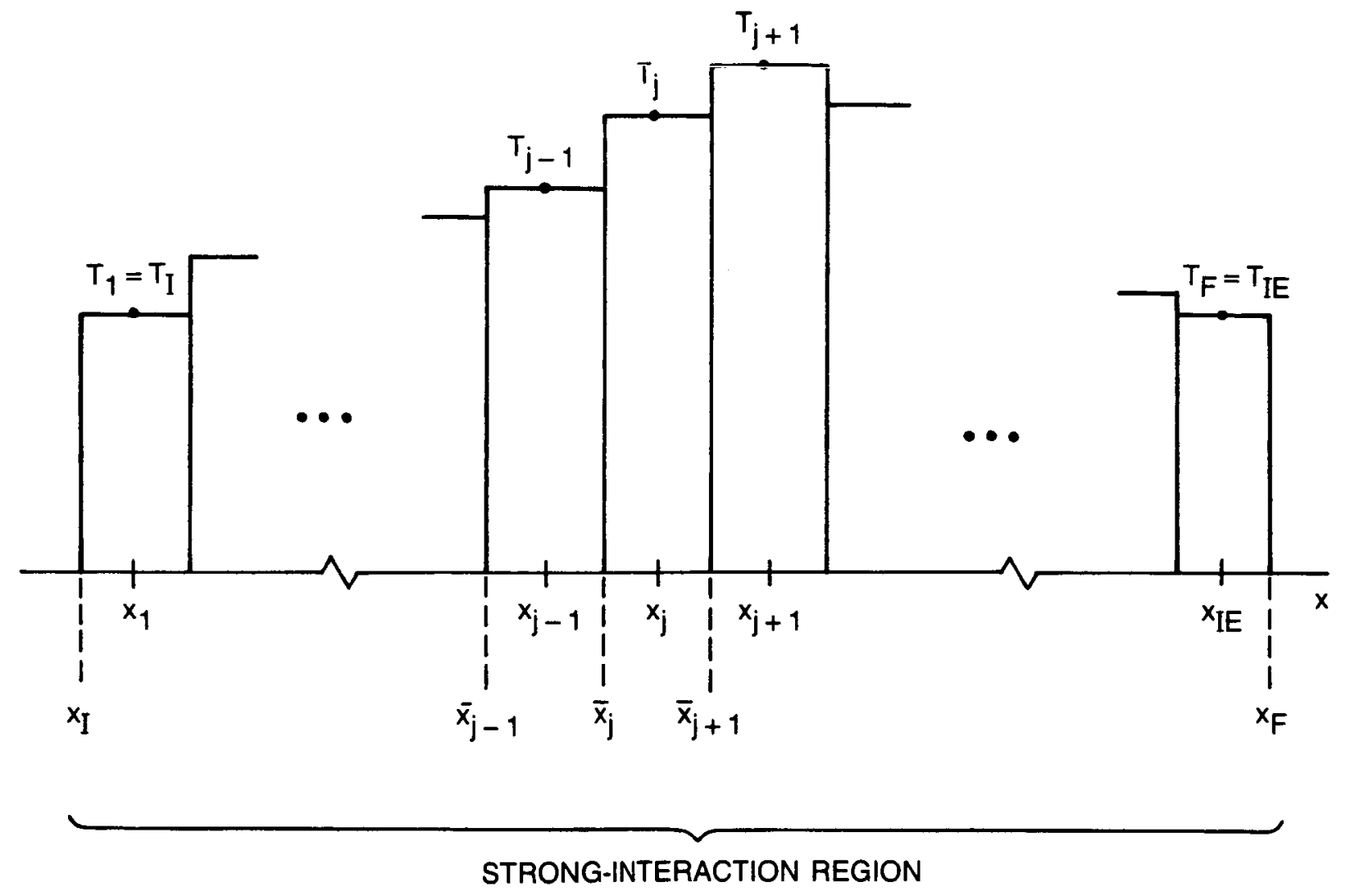

Fig. 5 Nomenclature for Inviscid Finite-Difference Mesh 


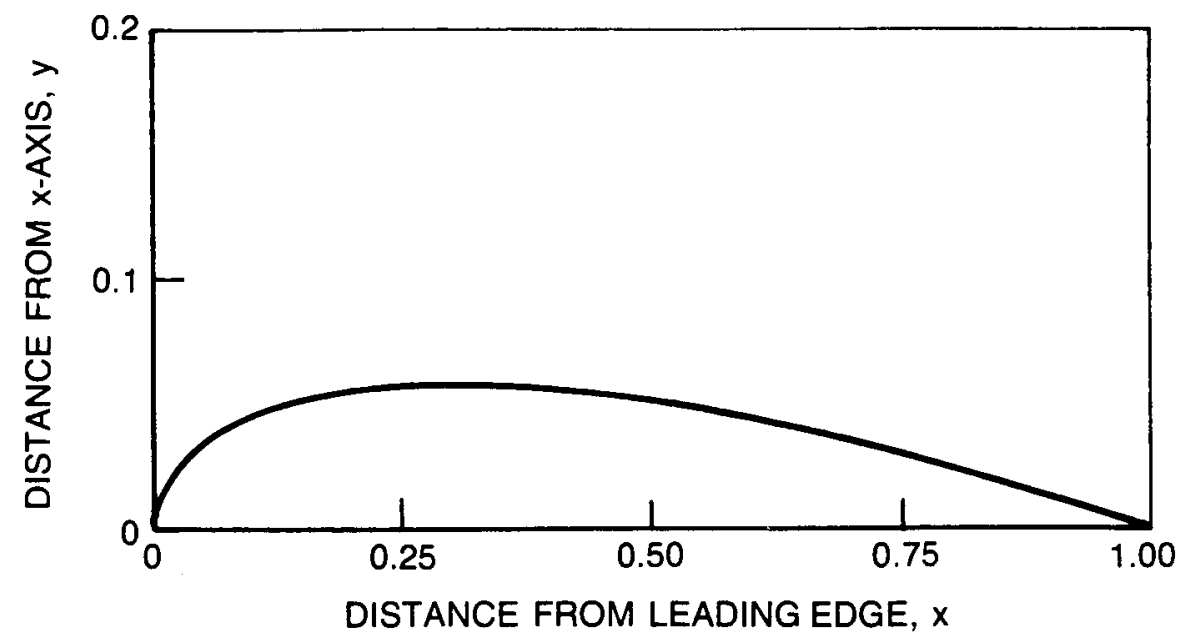

Fig. 6a NACA 0012 Airfoil Profile 


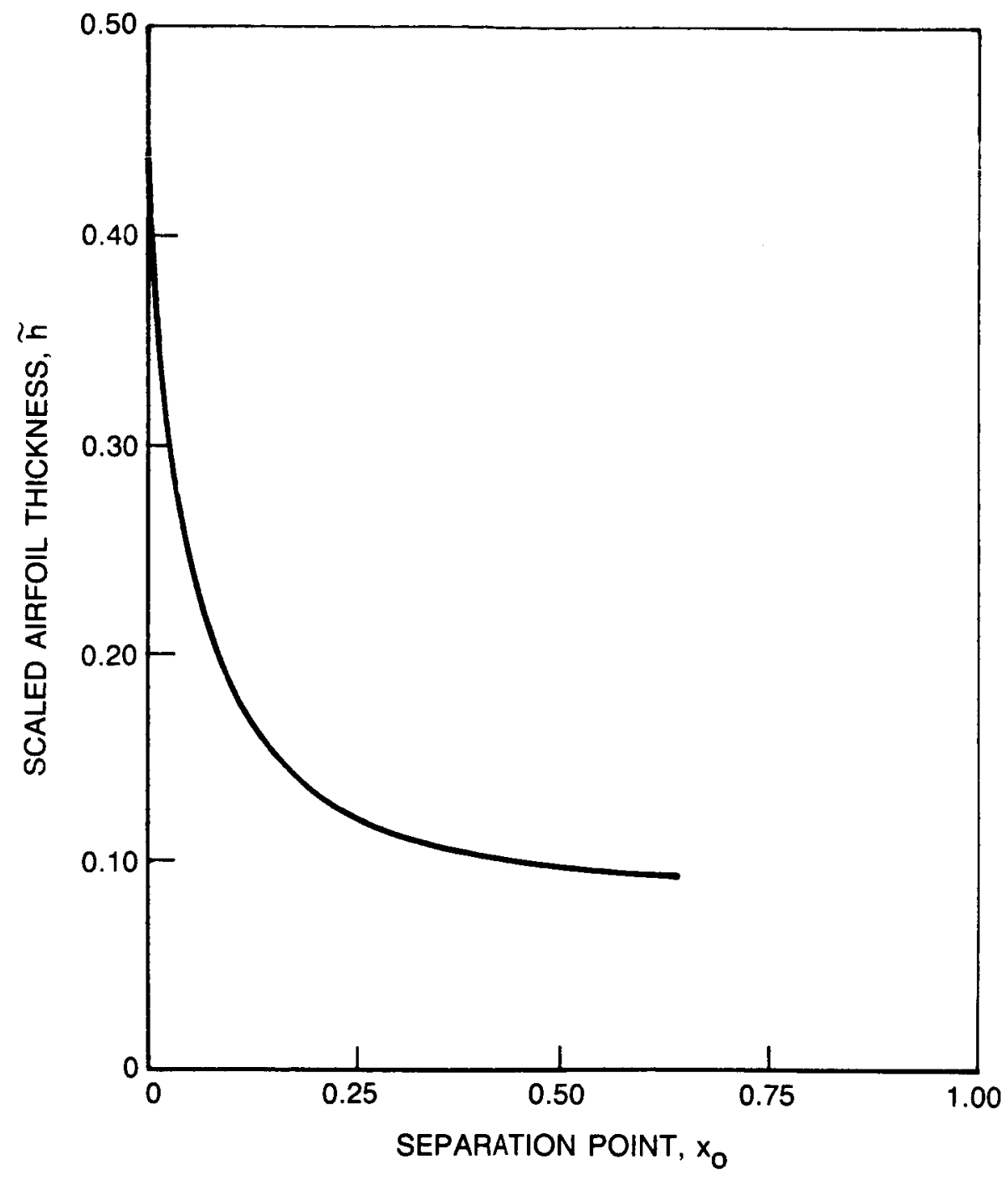

Fig. 6b Asymptotic Behavior of Separation Point Location for NACA 00XX Airfoil 


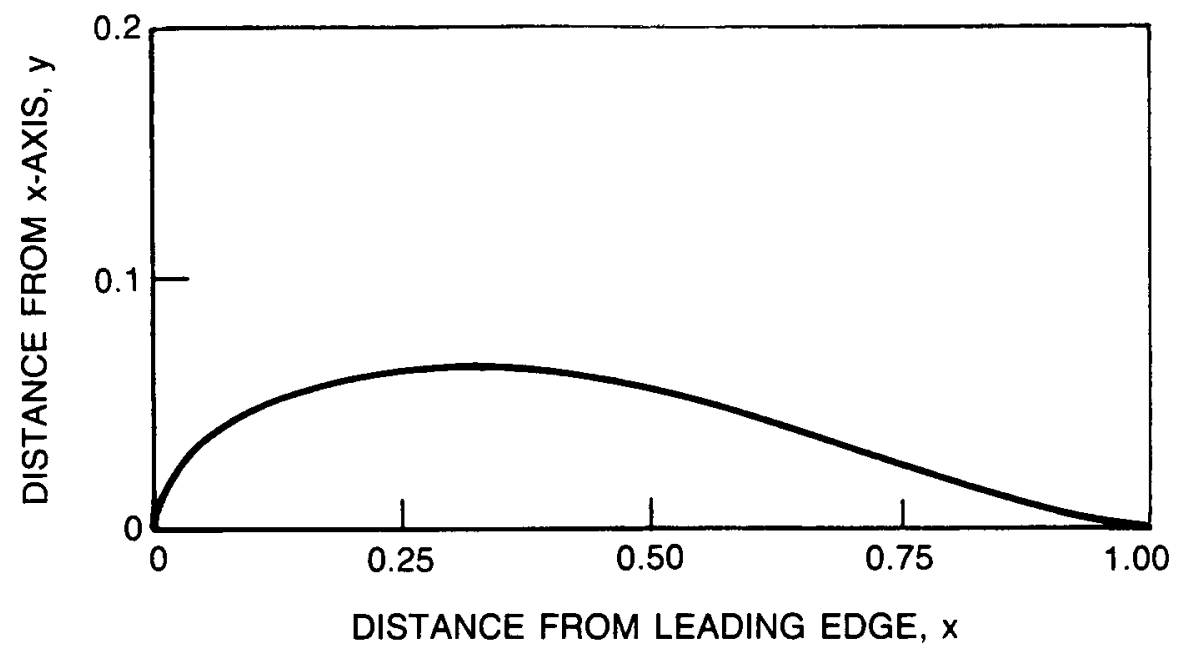

Fig. 7a Garabedian and Korn 70-10-13 Airfoil Profile 


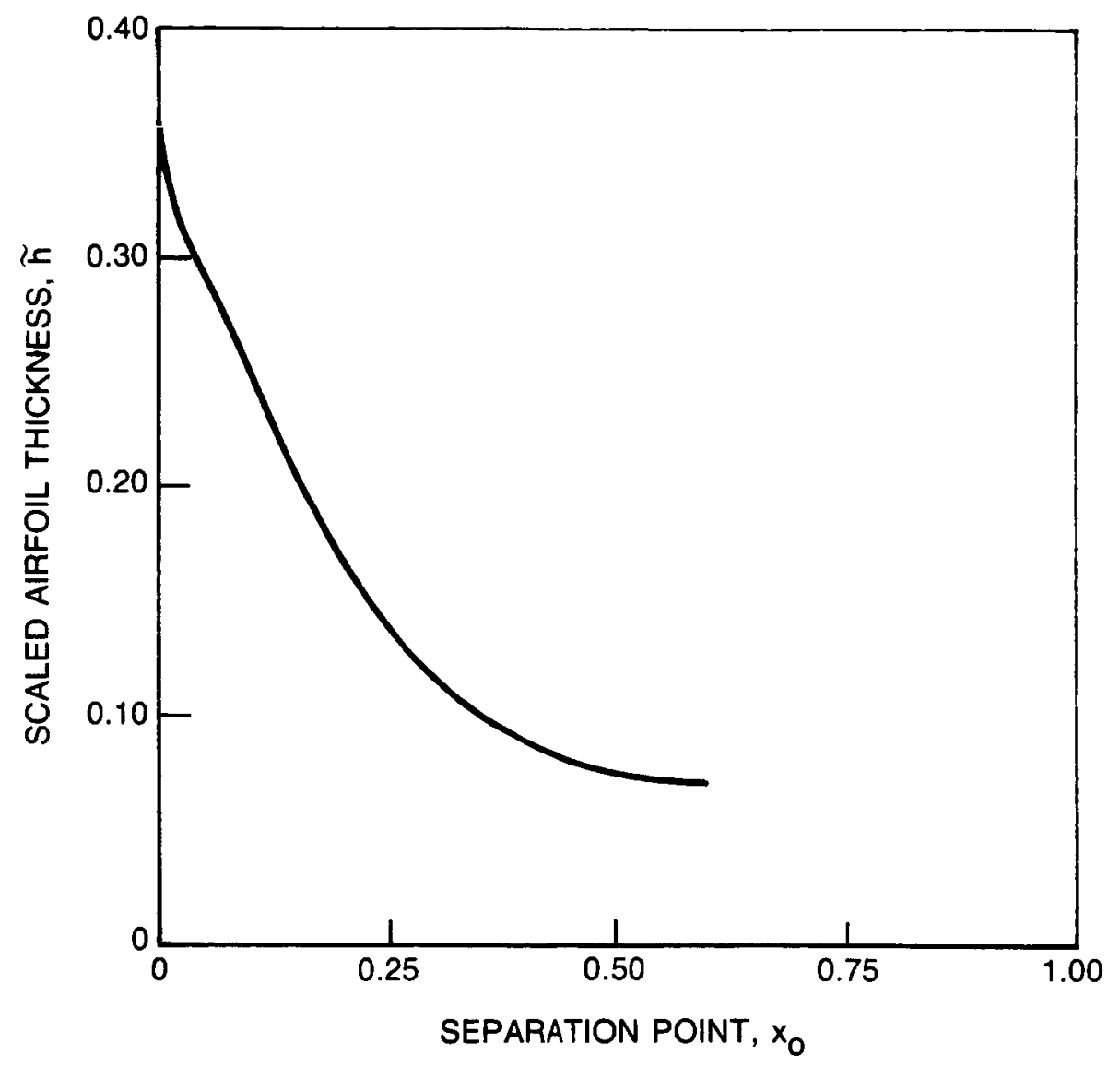

Fig. 7b Asymptotic Behavior of Separation Point Location for GK 70-10-XX Airfoil 


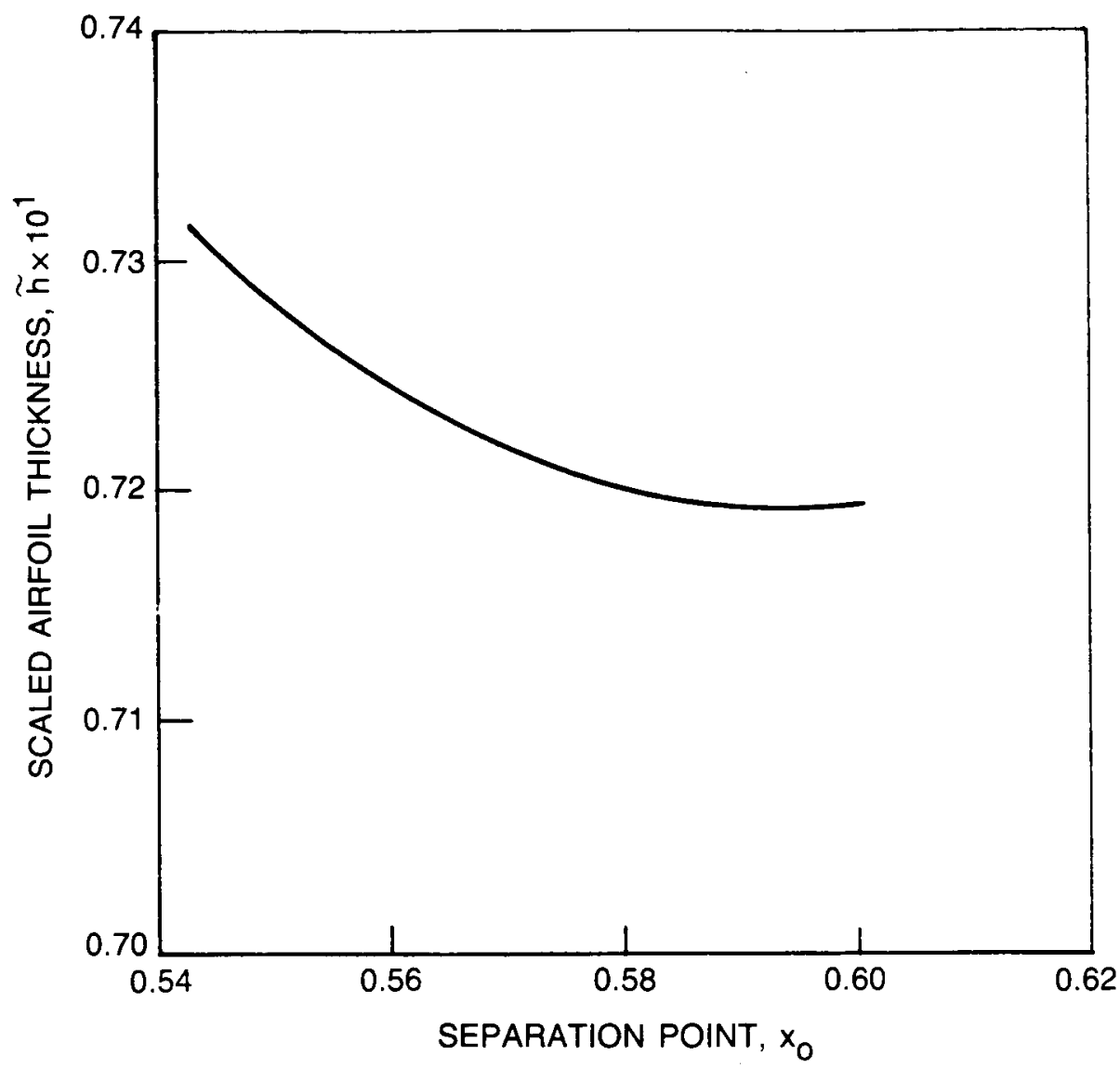

Fig. 7c Detail of Asymptotic Behavior of Separation Point Location for GK 70-10-XX Airfoil 


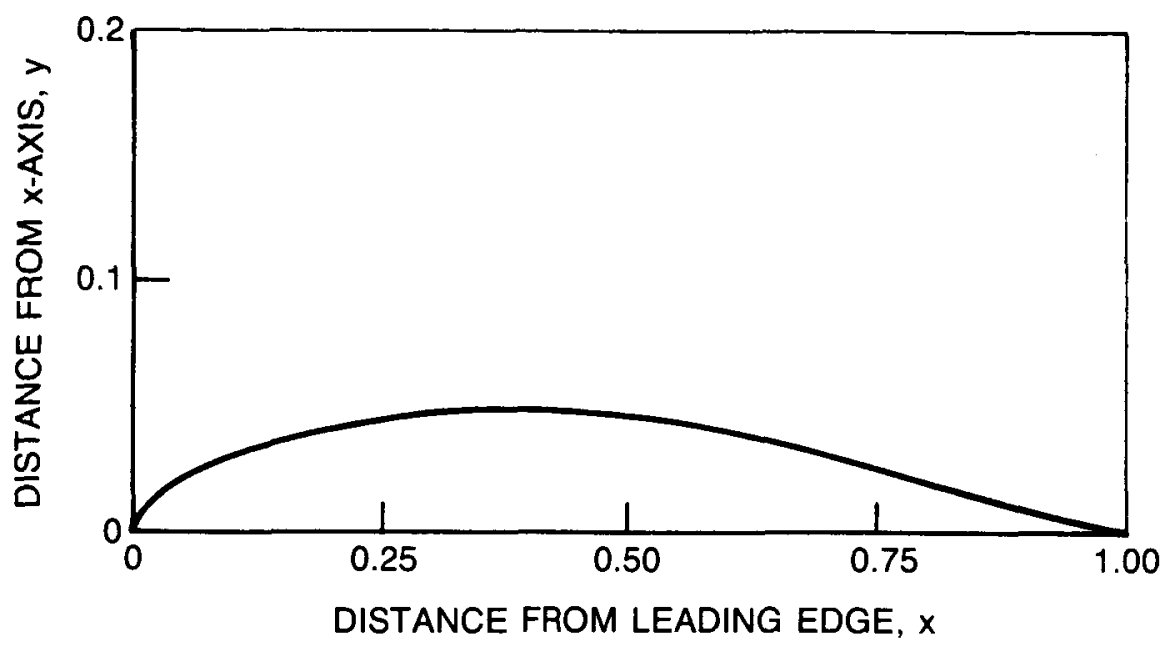

Fig. 8a NACA 65-010 Airfoil Profile 


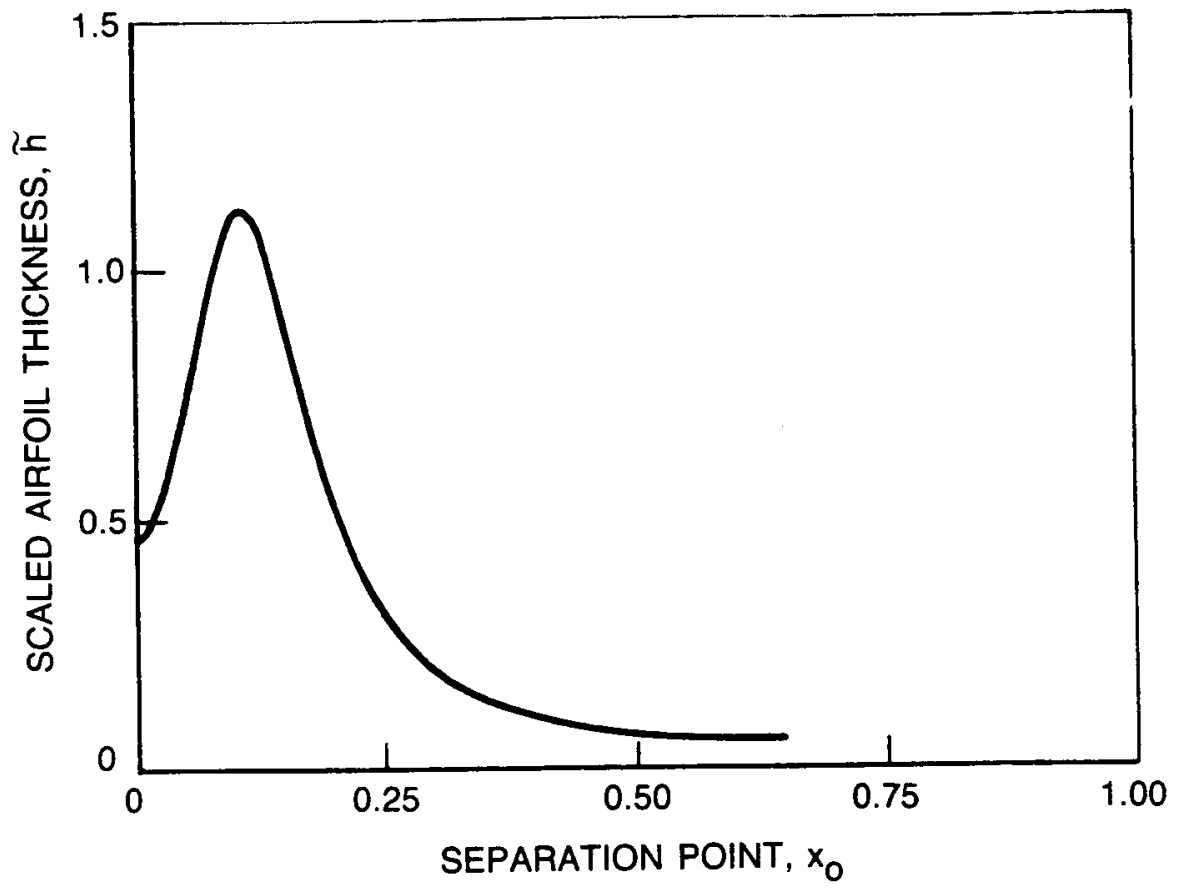

Fig. 8b Asymptotic Behavior of Separation Point Location for NACA 65-0XX Airfoil 


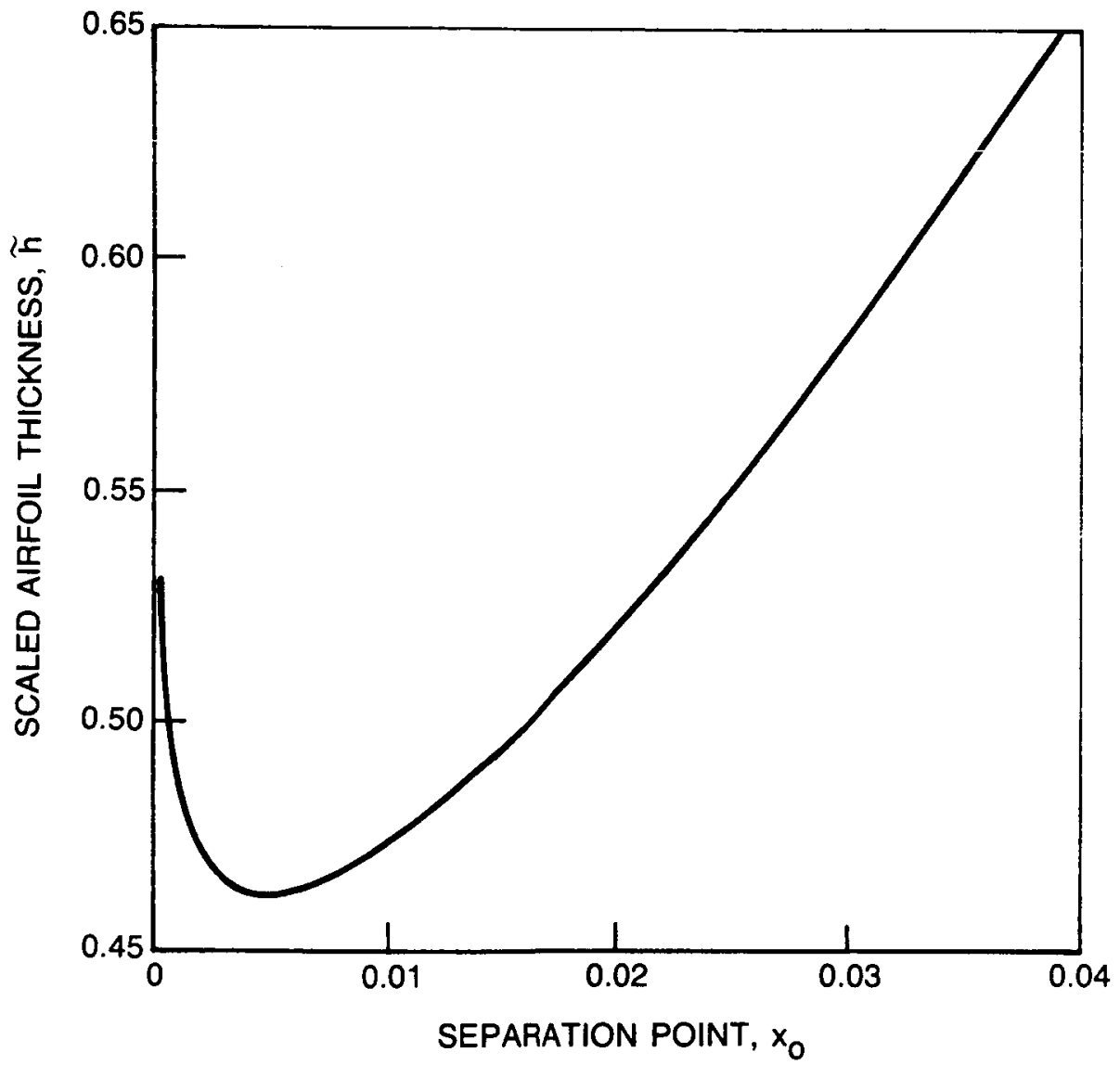

Fig. 8c Detail of Asymptotic Behavior of Separation Point Location for NACA 65-0XX Airfoil 


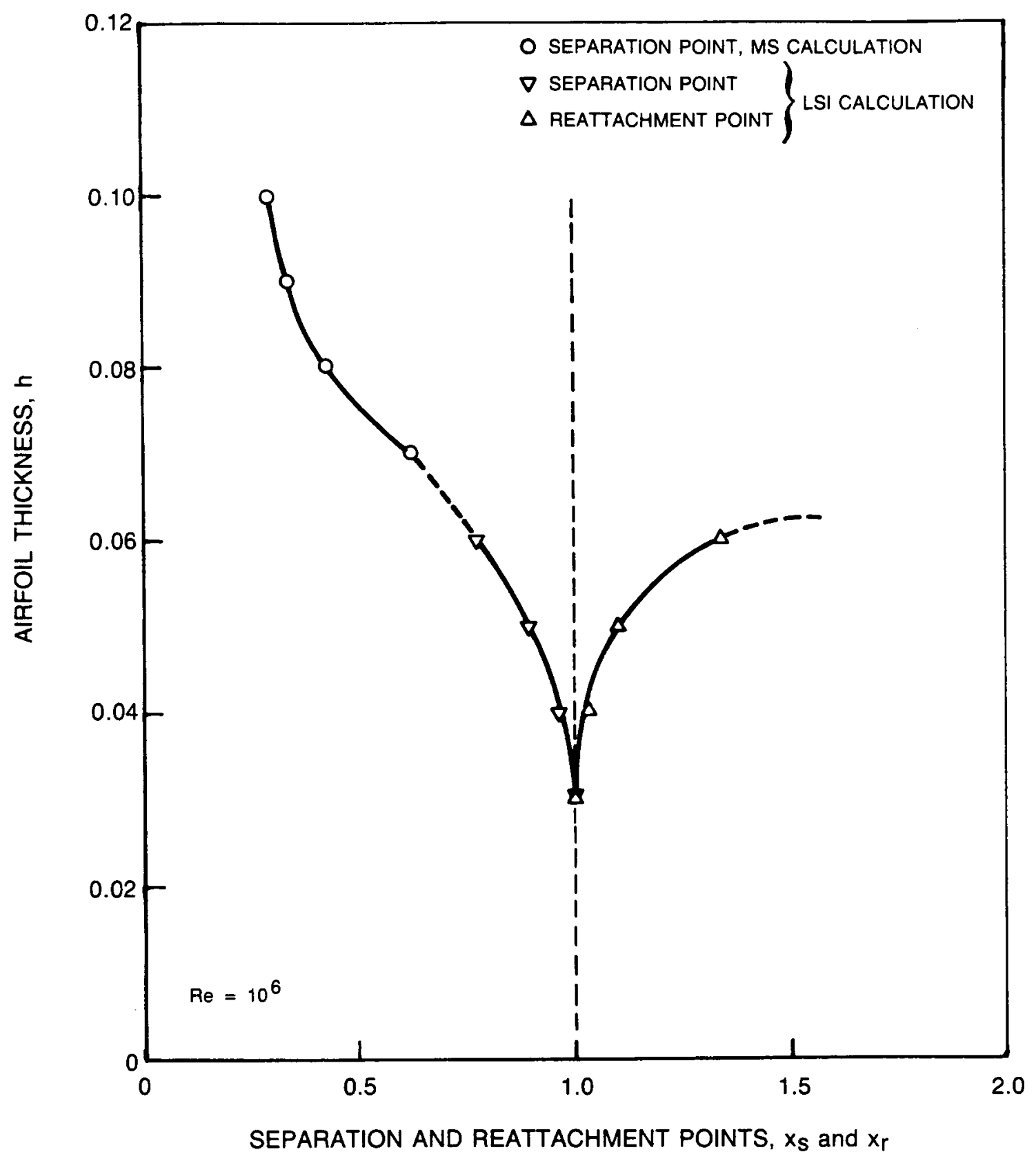

Figure 9 Behavior of Separation and Reattachment Point Locations for NACA 00XX Airfoil 


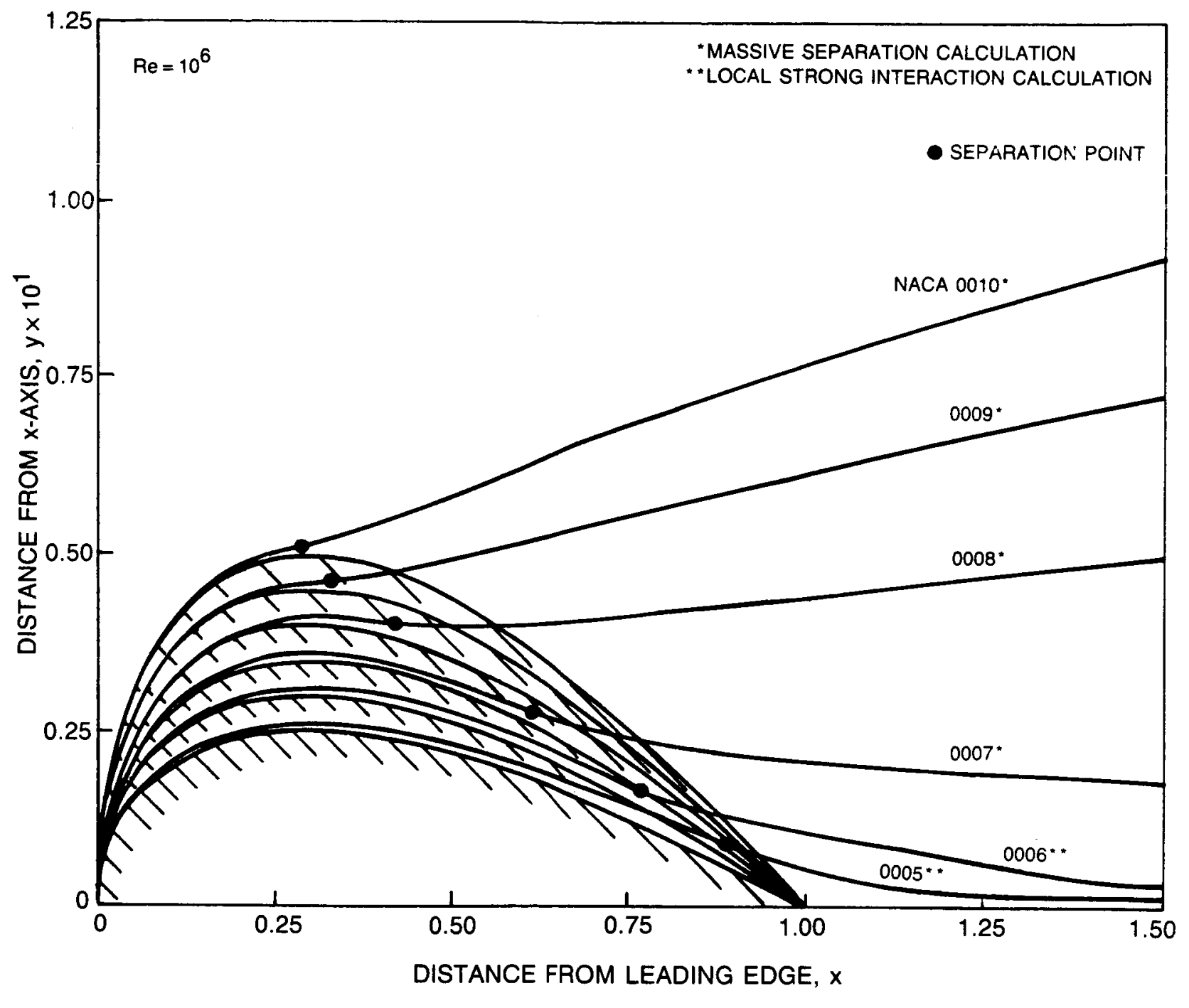

Fig. 10a Airfoil Profile and Displacement Body for NACA 00XX Airfoil 


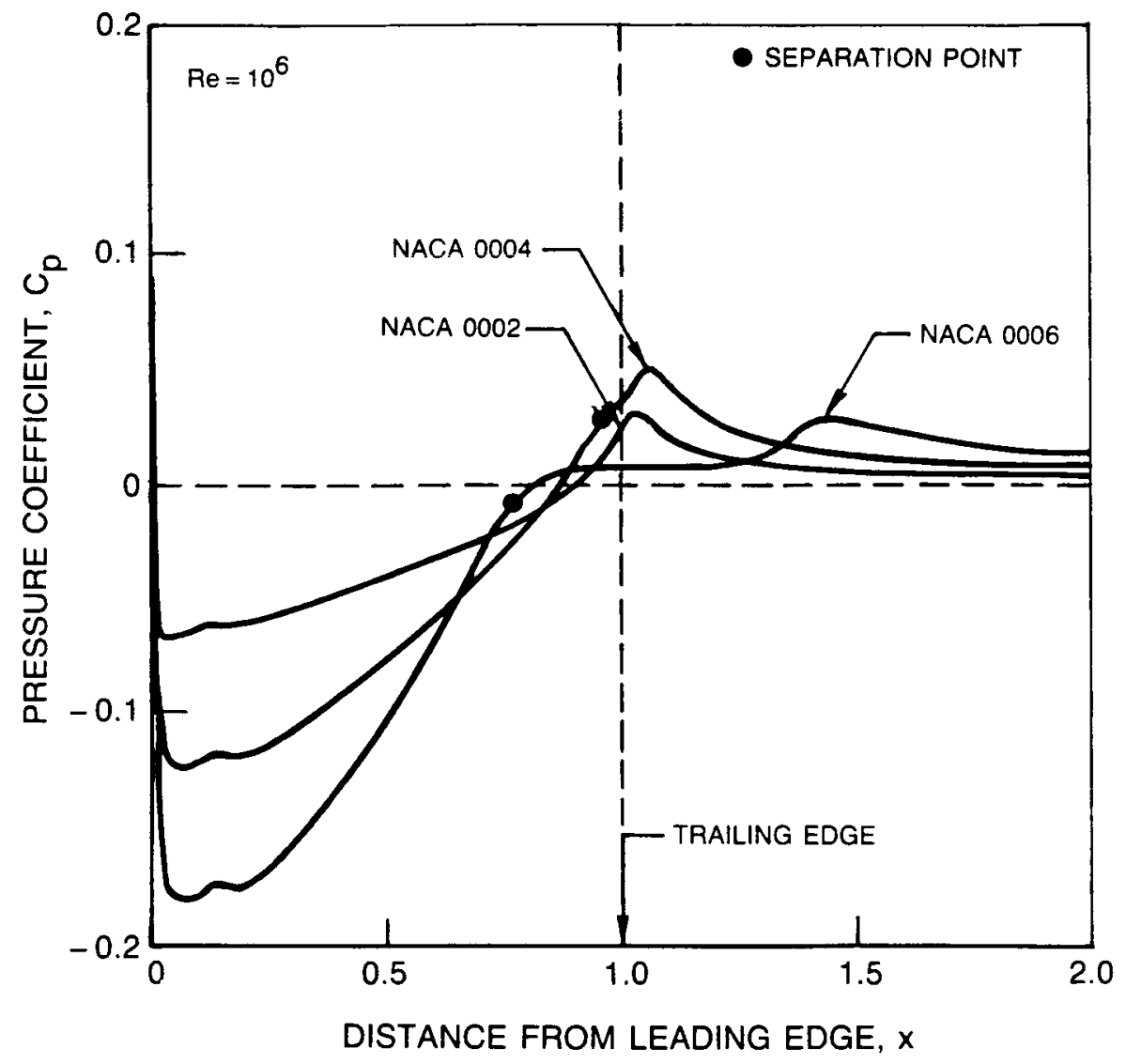

Fig. 10b Pressure Distribution on Airfoil Surface and Wake Centerline for NACA 00XX Airfoil Using LSI Method 


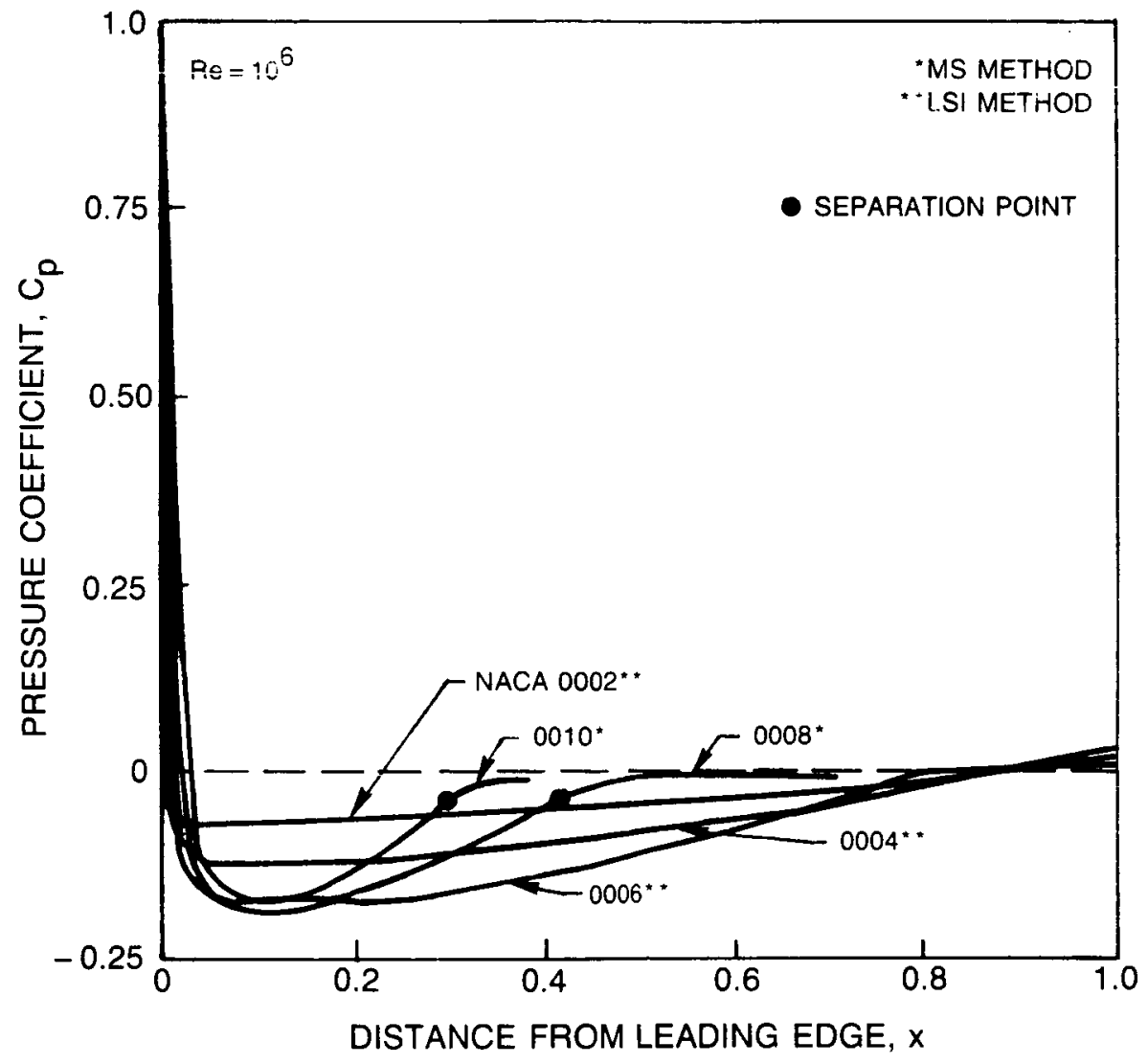

Fig. 10c Pressure Distribution on Airfoil Surface for NACA 00XX Airfoil Using LSI and MS Methods 


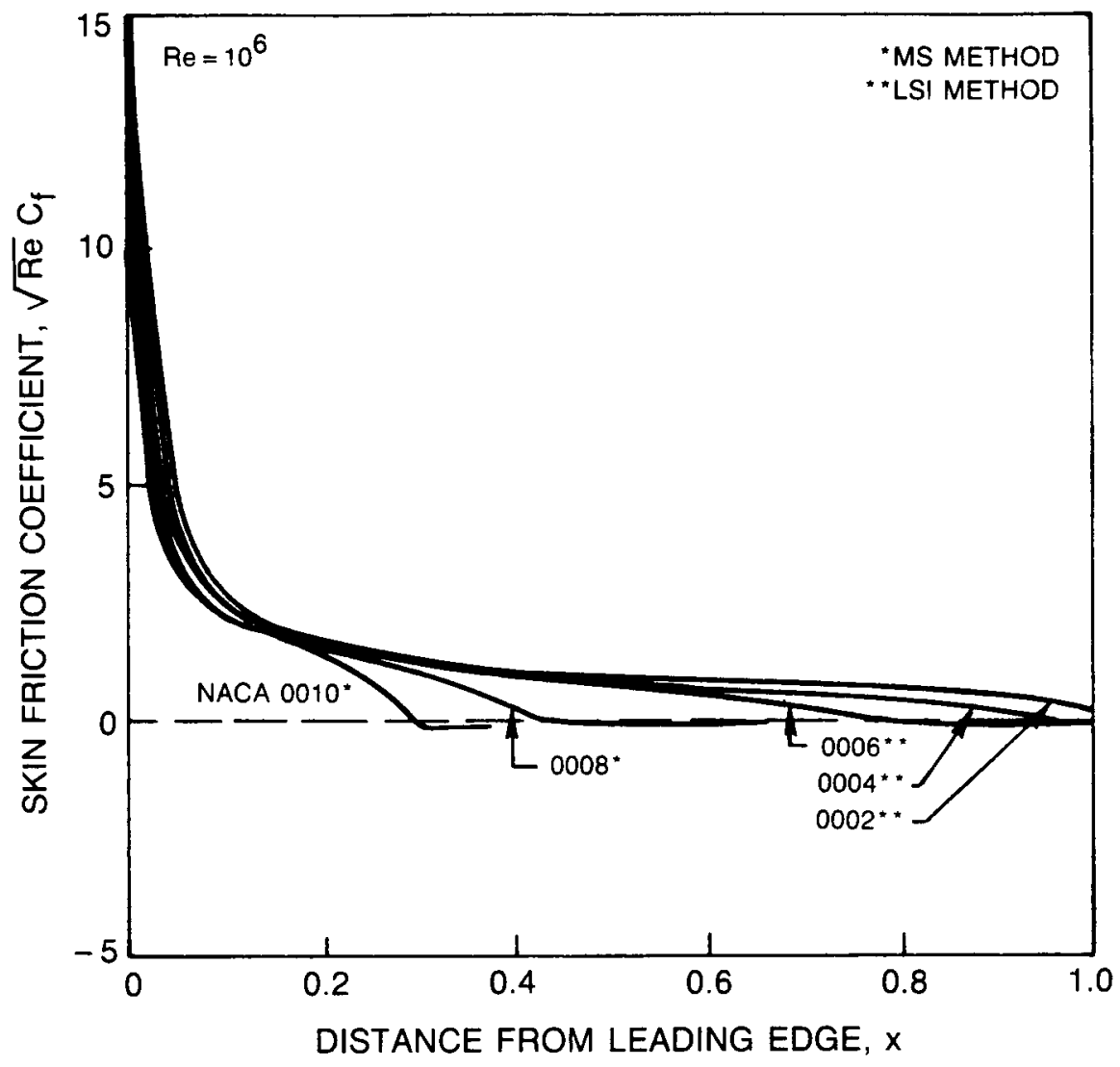

Fig. 10d Skin Friction Distribution for NACA 00XX Airfoil Using LSI and MS Methods 


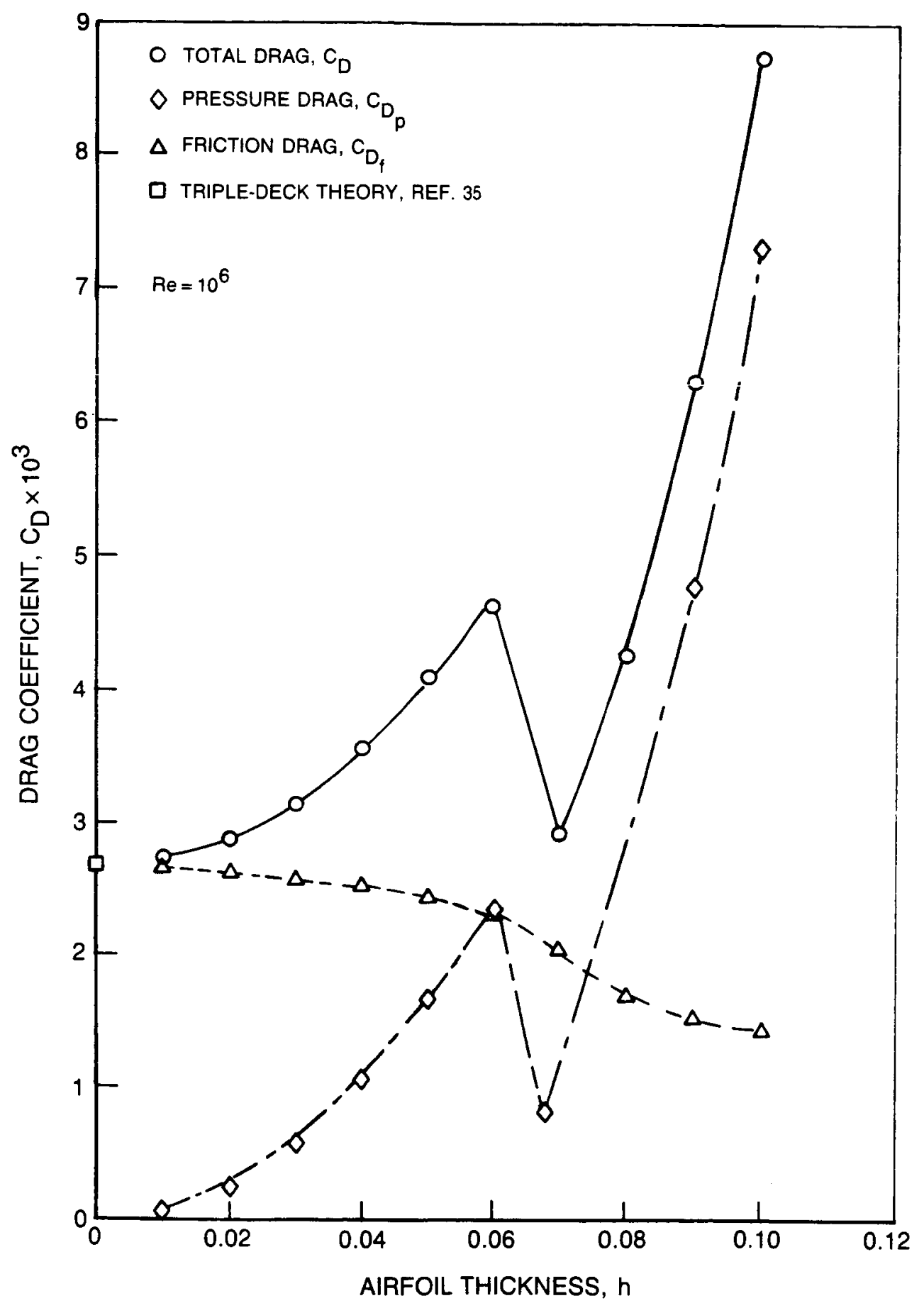

Fig. 11 Drag as Function of Airfoil Thickness for NACA 00XX Airfoil 


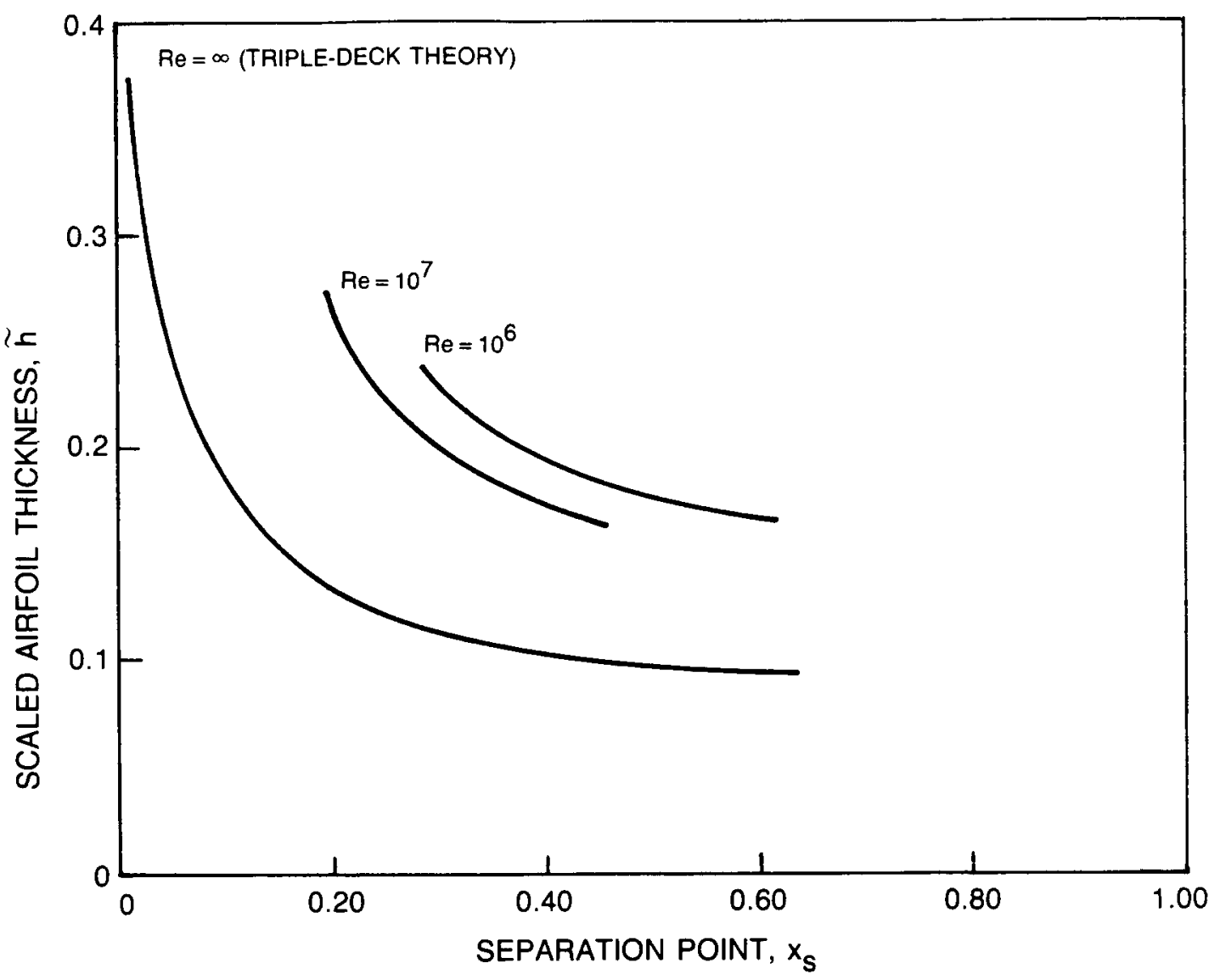

Fig. 12 Effect of Reynolds Number on Separation Point Location for NACA 00XX Airfoil 


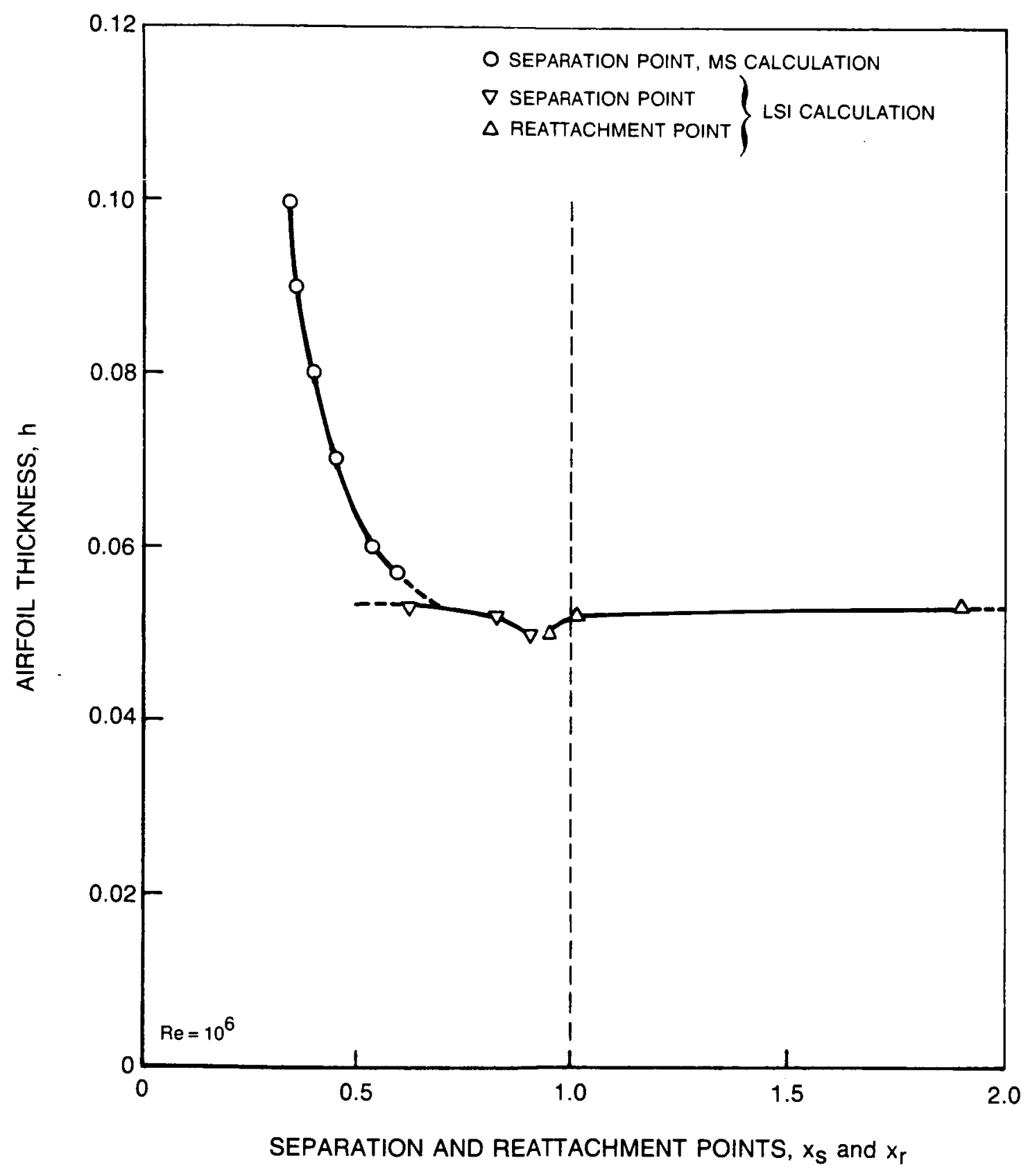

Figure 13 Behavior of Separation and Reattachment Point Locations for GK 70-10-XX Airfoil 


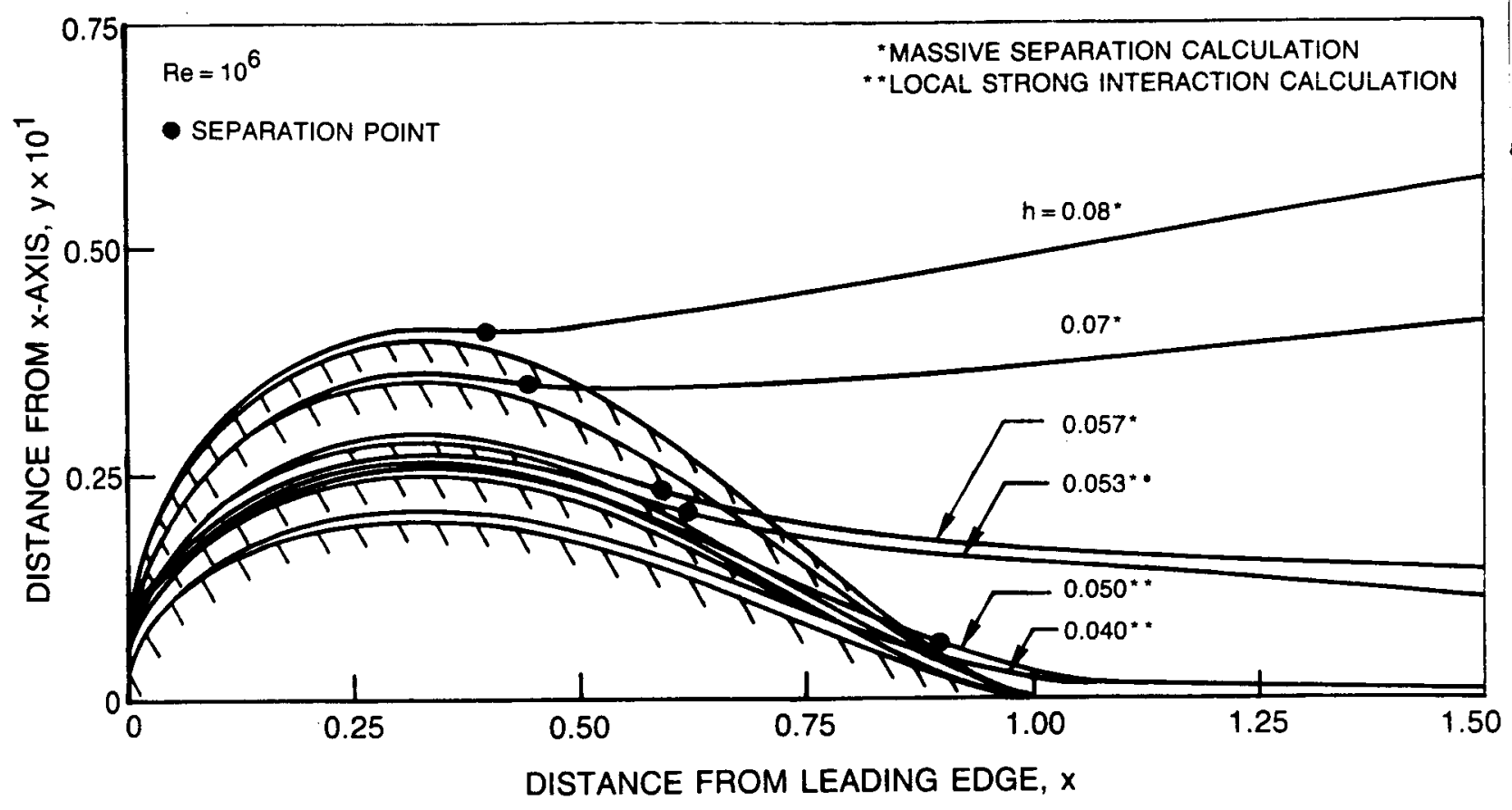

Fig. 14a Airfoil and Displacement Body for GK 70-10-XX Airfoil 


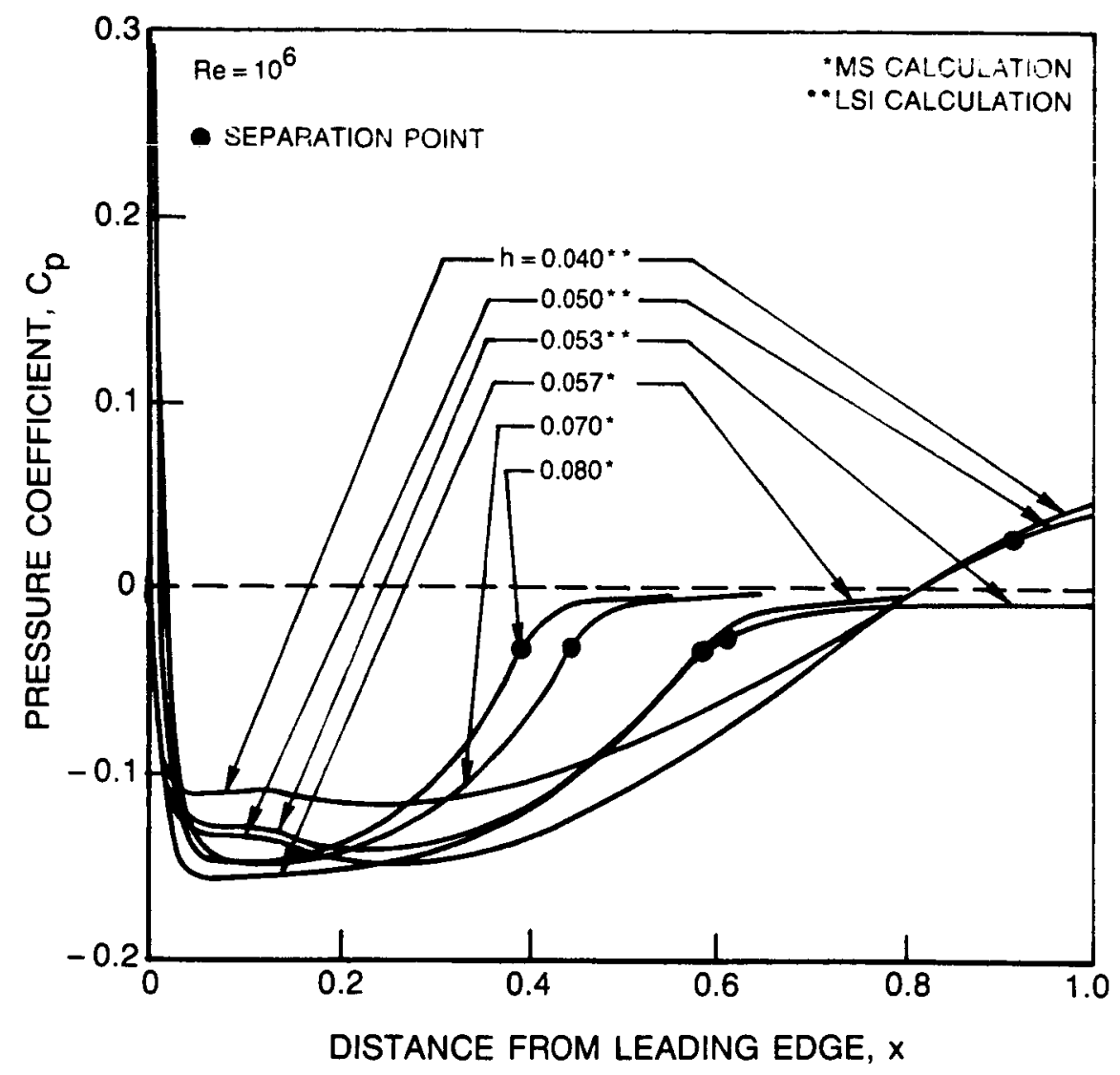

Fig. 14b Pressure Distribution for GK 70-10-XX Airfoil 


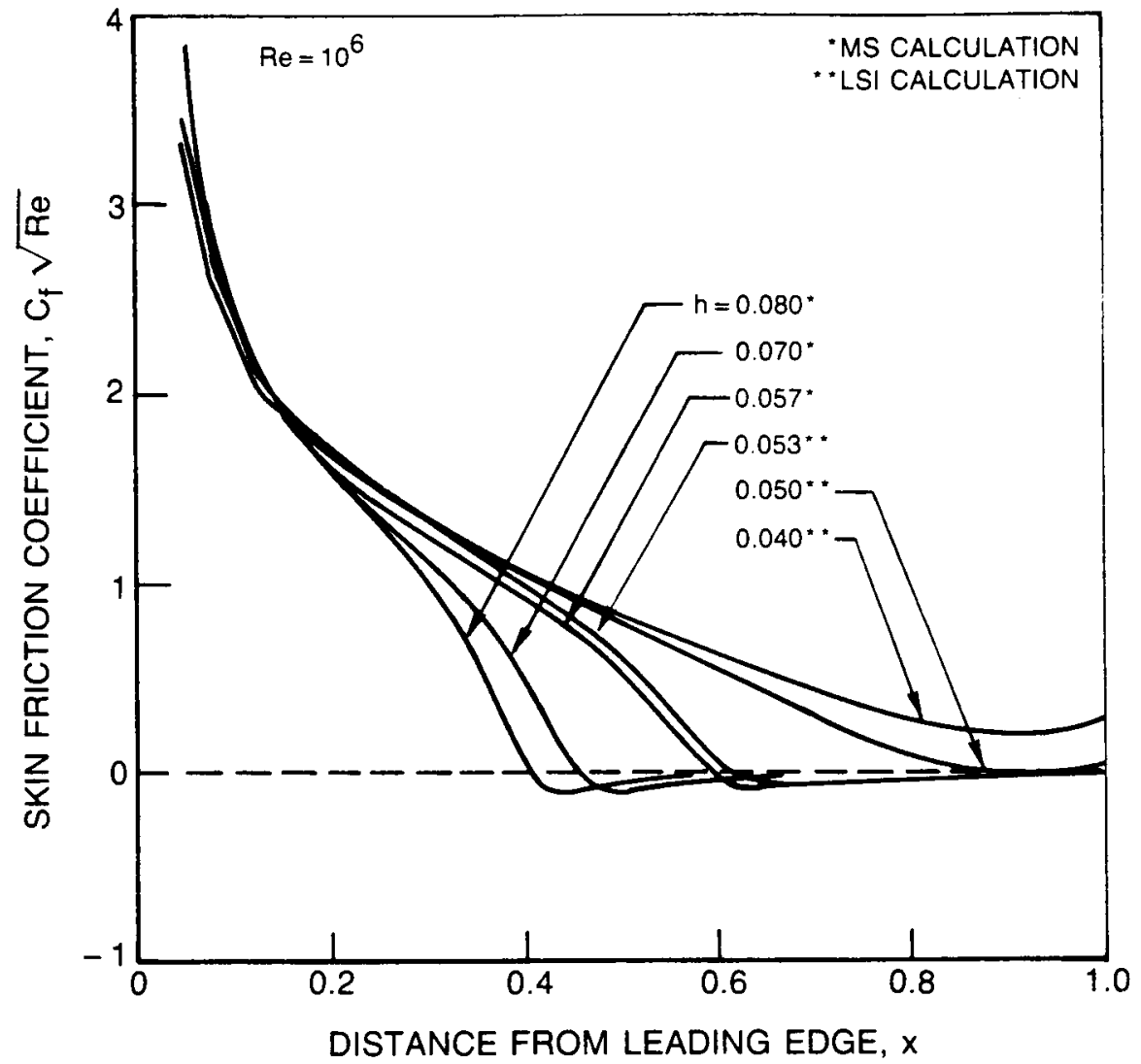

Fig. 14c Skin Friction Distribution for GK 70-10-XX Airfoil 


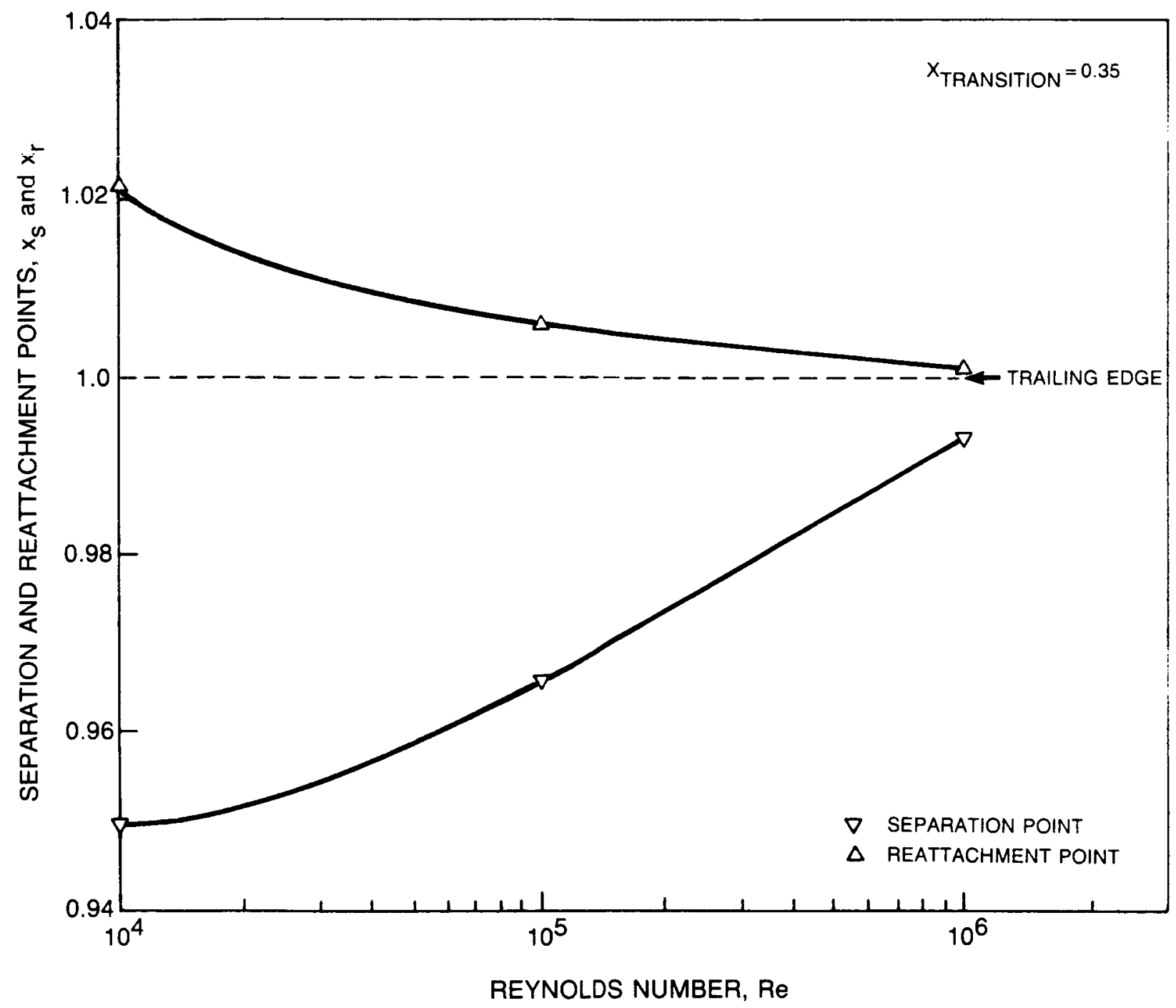

Fig. 15 Behavior of Separation and Reattachment Points in Turbulent Flow as Reynolds Number Varies for NACA 0014 Airfoil 


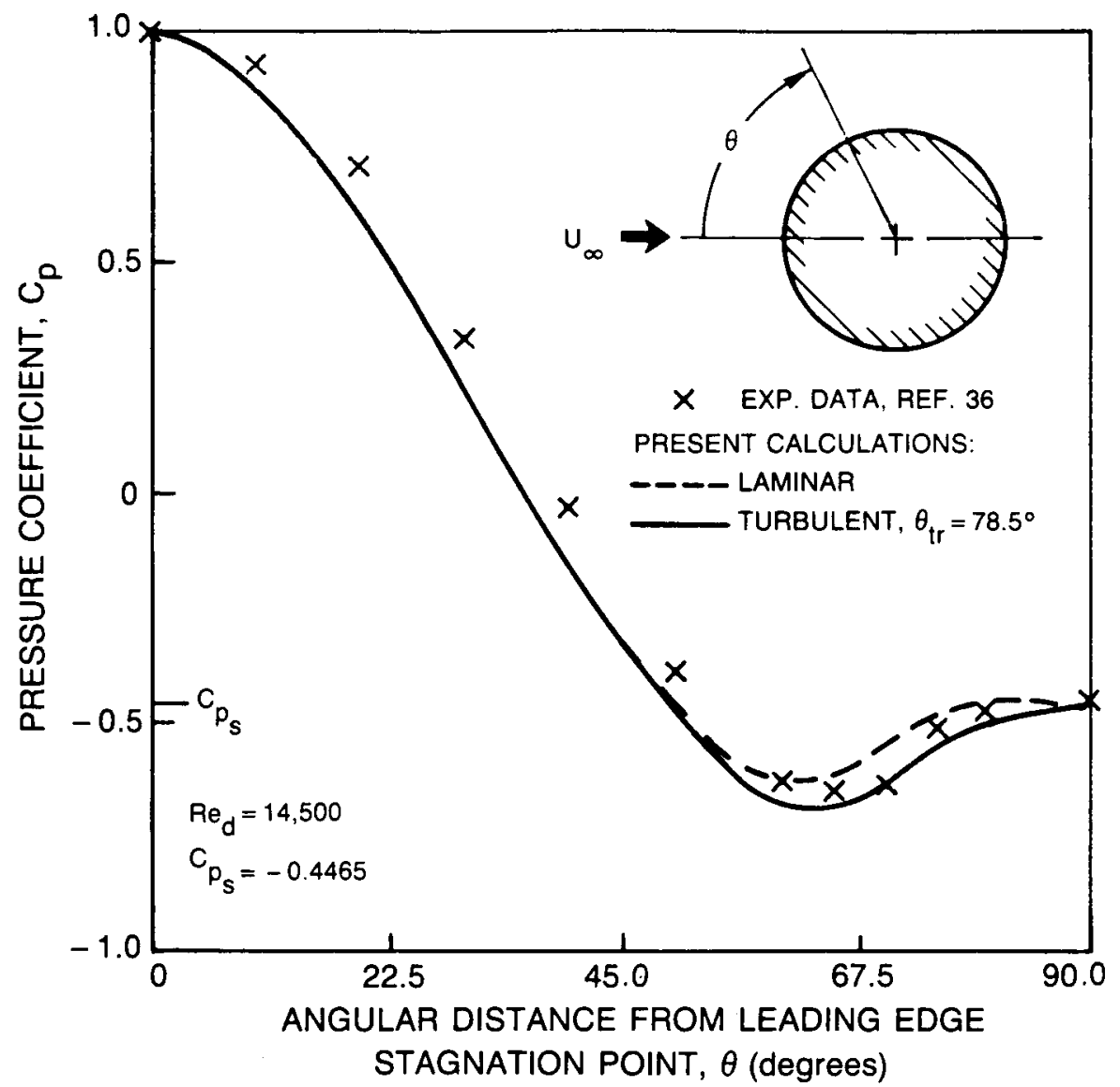

Fig. 16 Pressure Distributions for Laminar and Transitional Flow Past Circular Cylinder with Splitter Plate, Compared with Experimental Data 


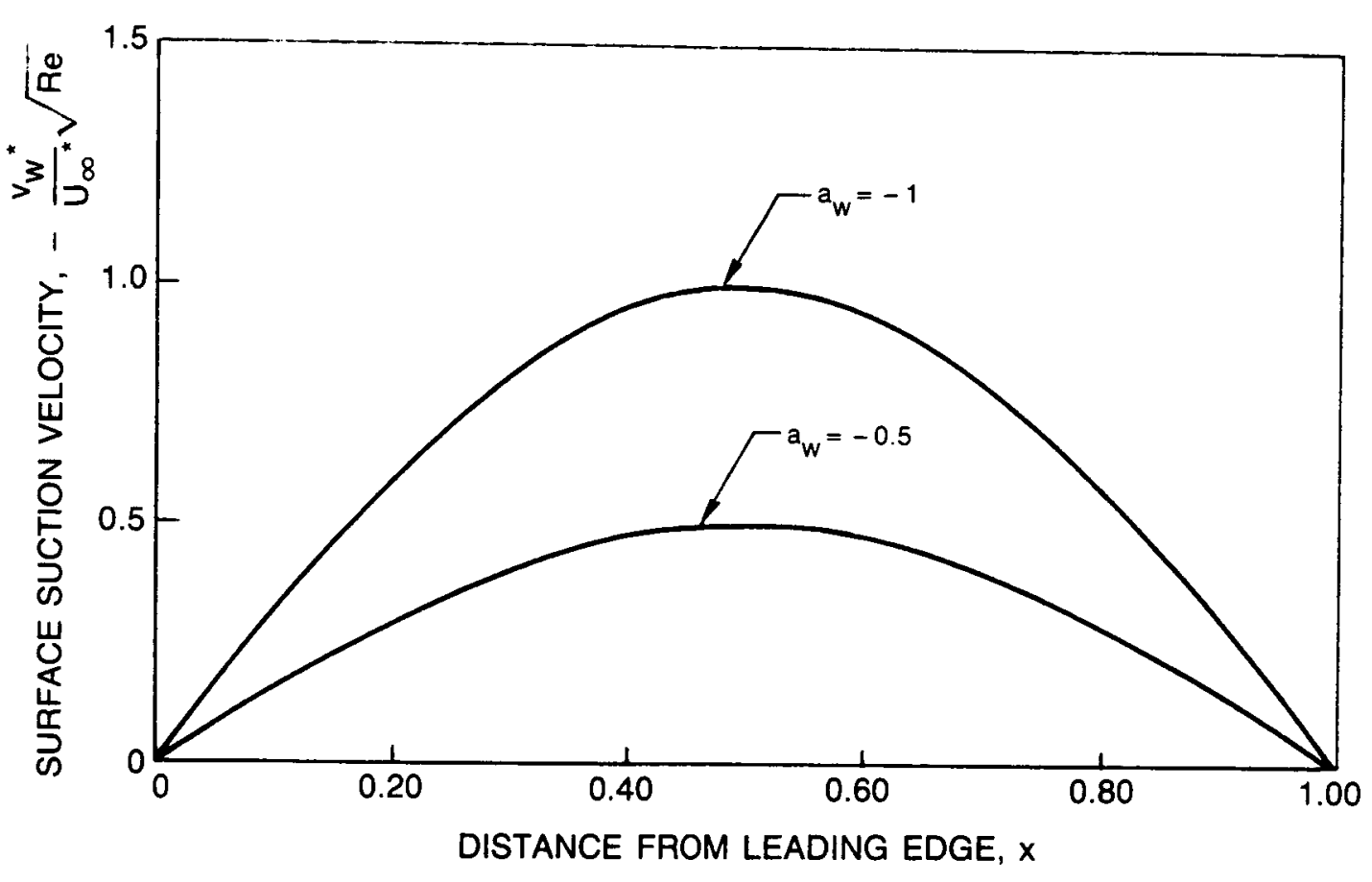

Fig. 17 Distribution of Full-Chord Suction for NACA 0006 Airfoil 


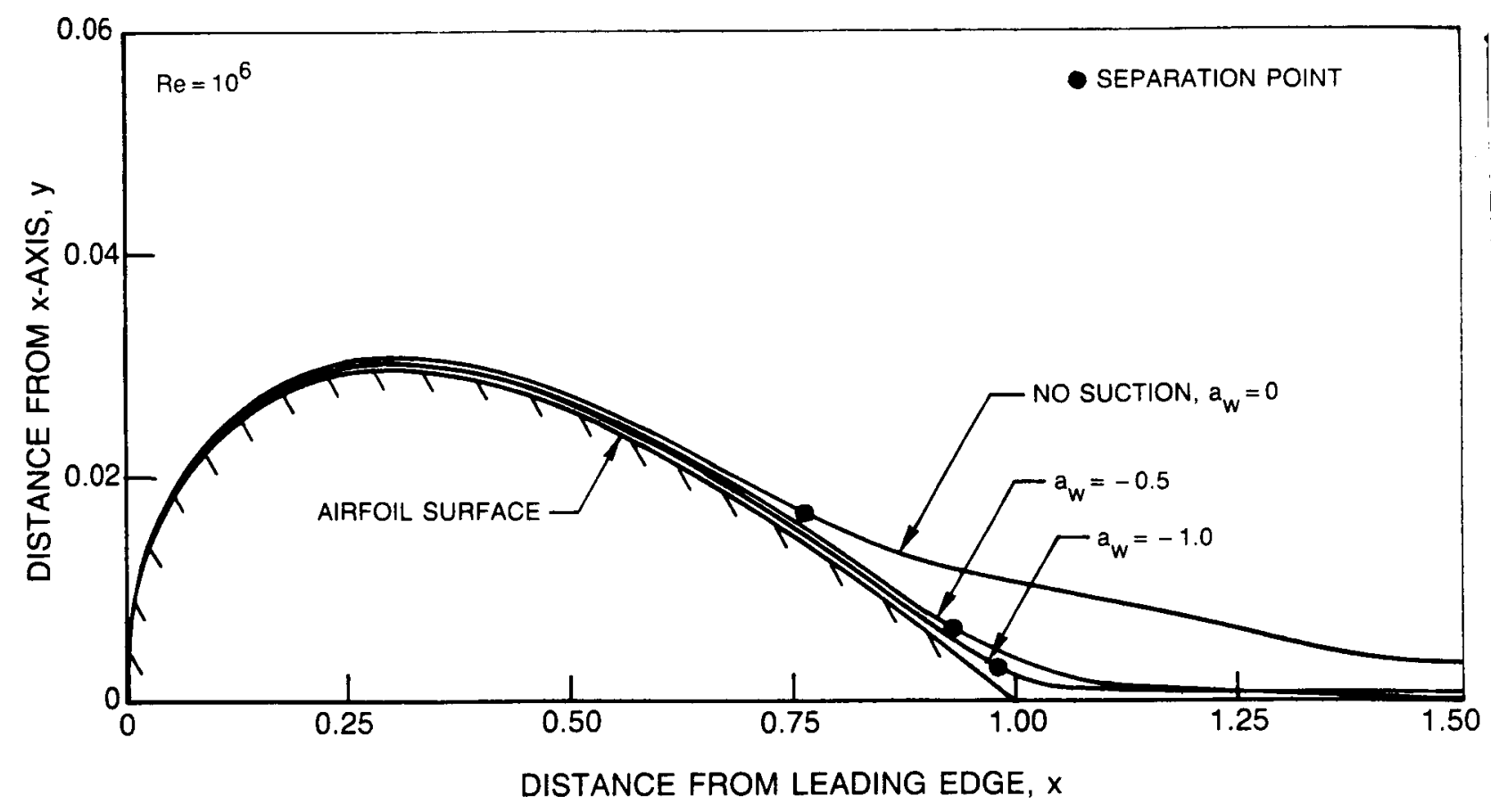

Fig. 18a Airfoil Profile and Displacement Body for NACA 0006 Airfoil with Full-Chord Suction 


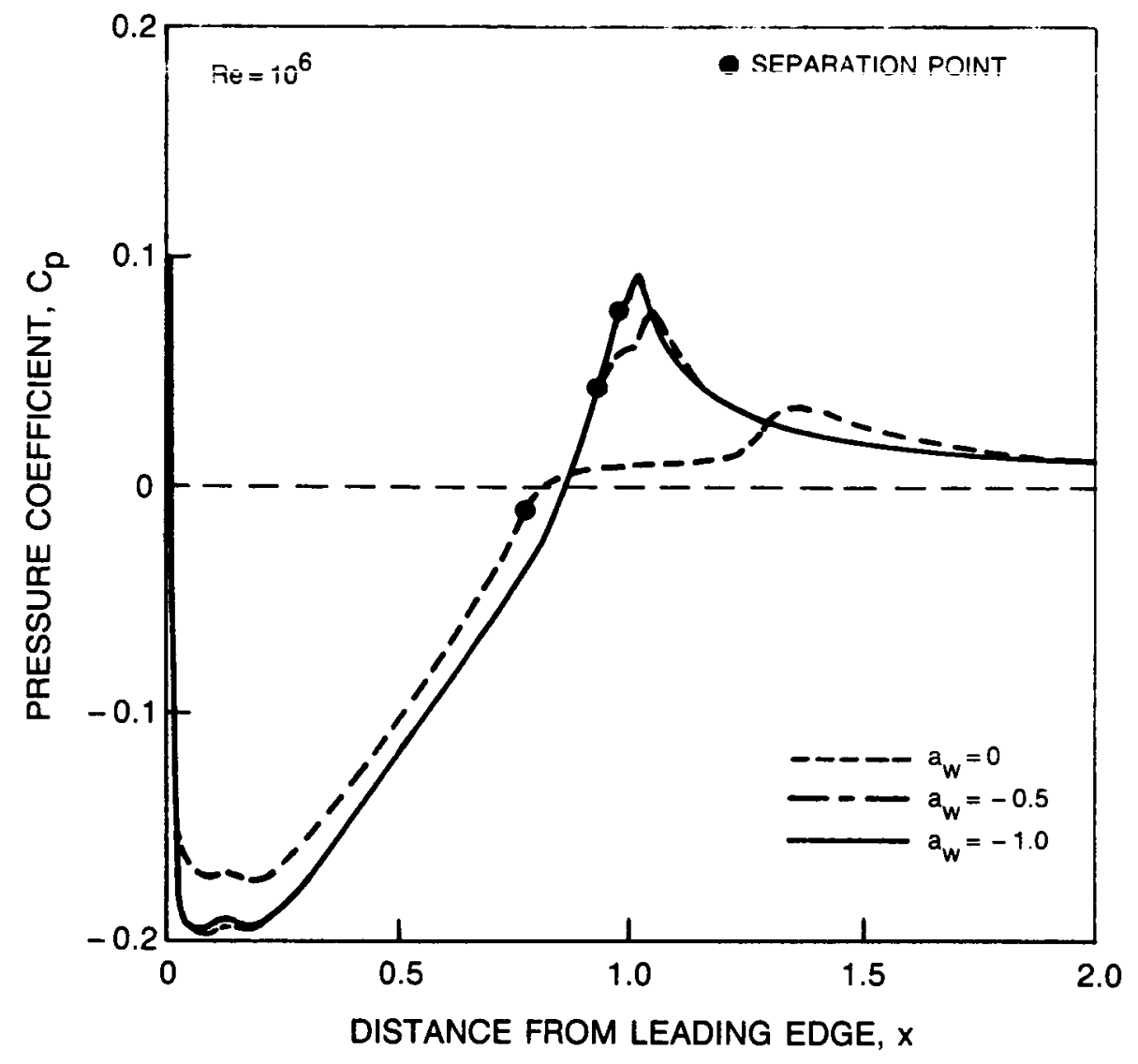

Fig. 18b Pressure Distribution for NACA 0006 Airfoil with Full-Chord Suction 


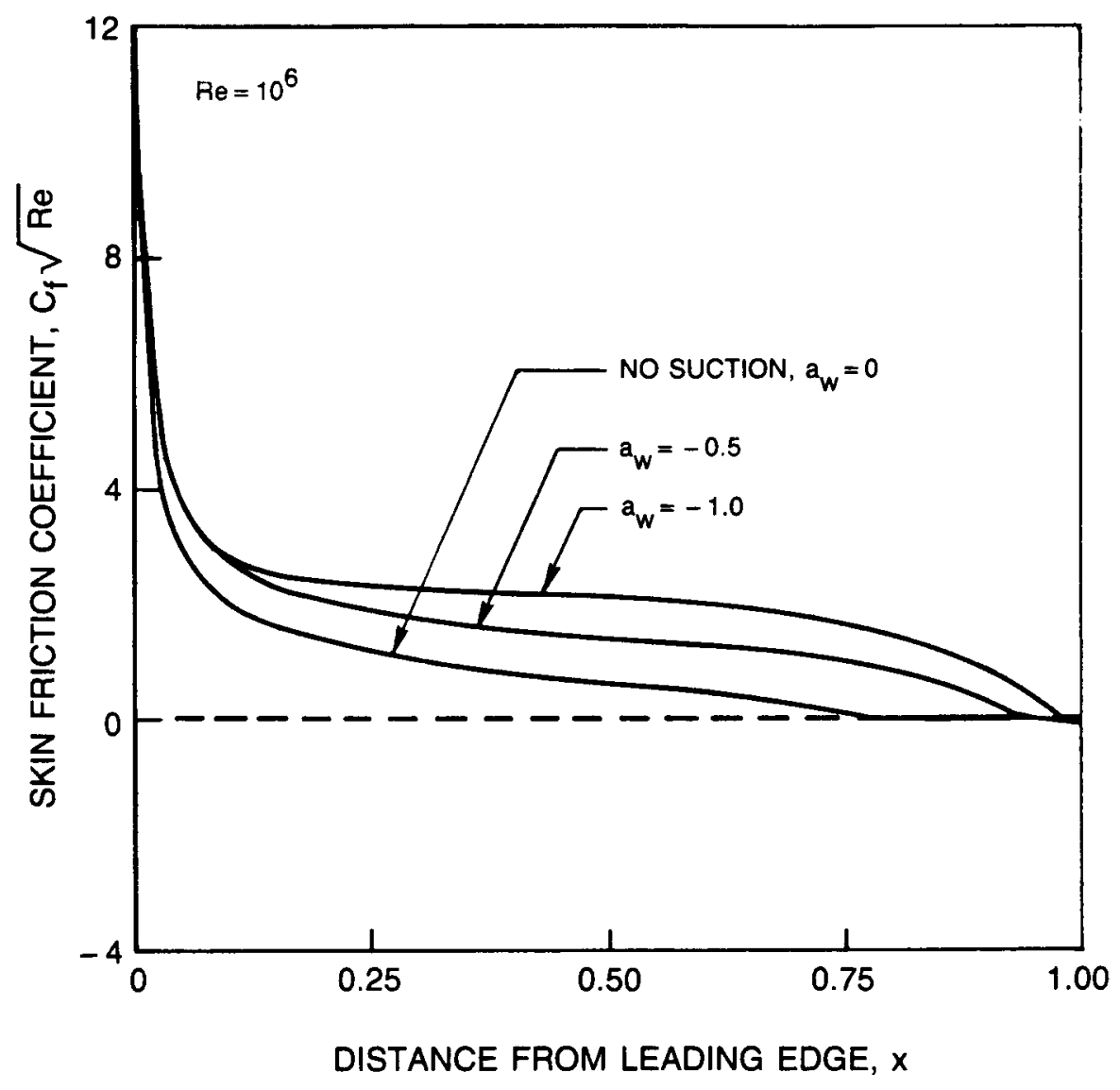

Fig. 18c Skin Friction Distribution for NACA 0006 Airfoll with Full-Chord Suction 


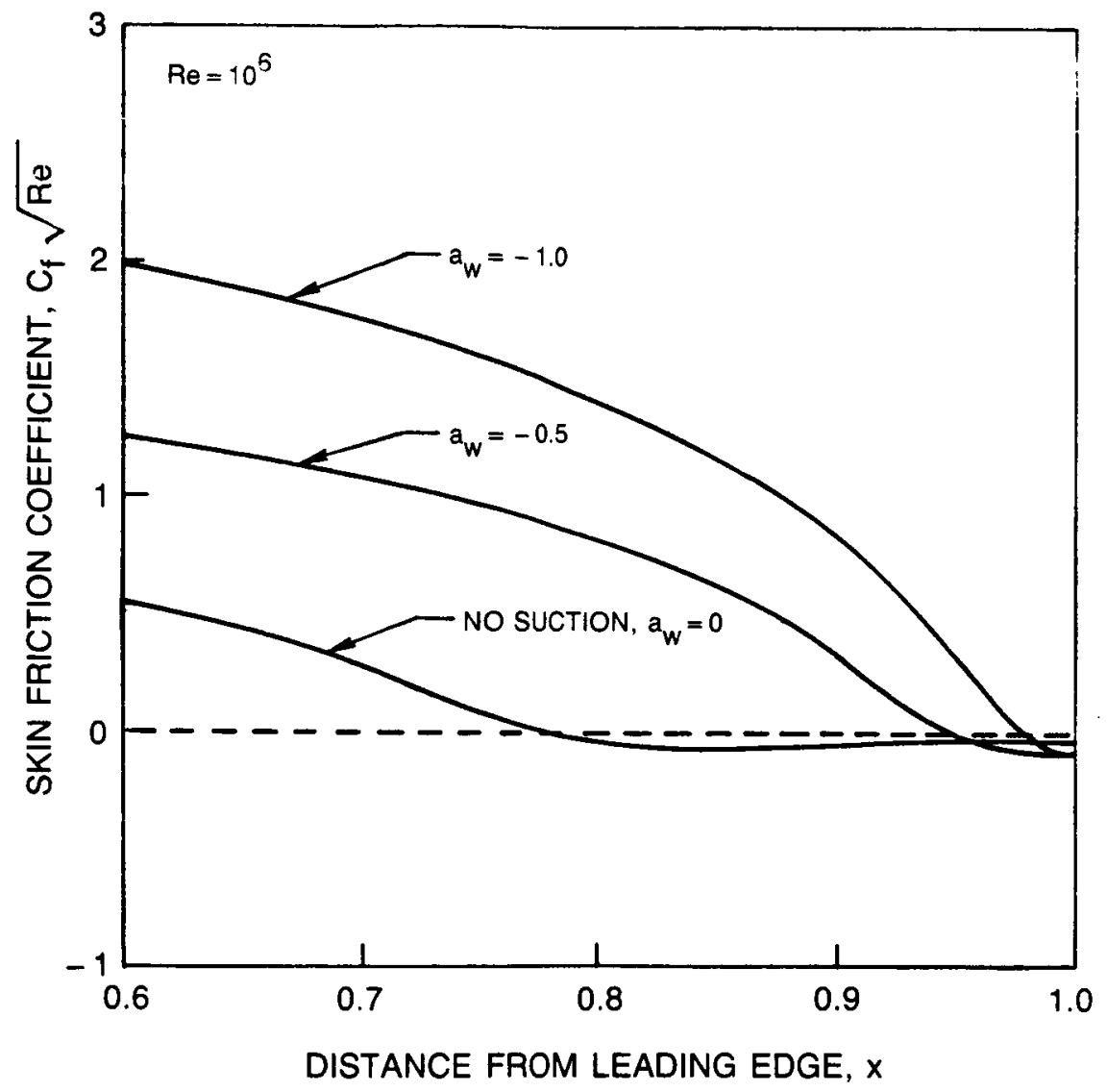

Fig. 18d Skin Friction Distribution in Trailing-Edge Region of NACA 0006 Airfoil with Full-Chord Suction 


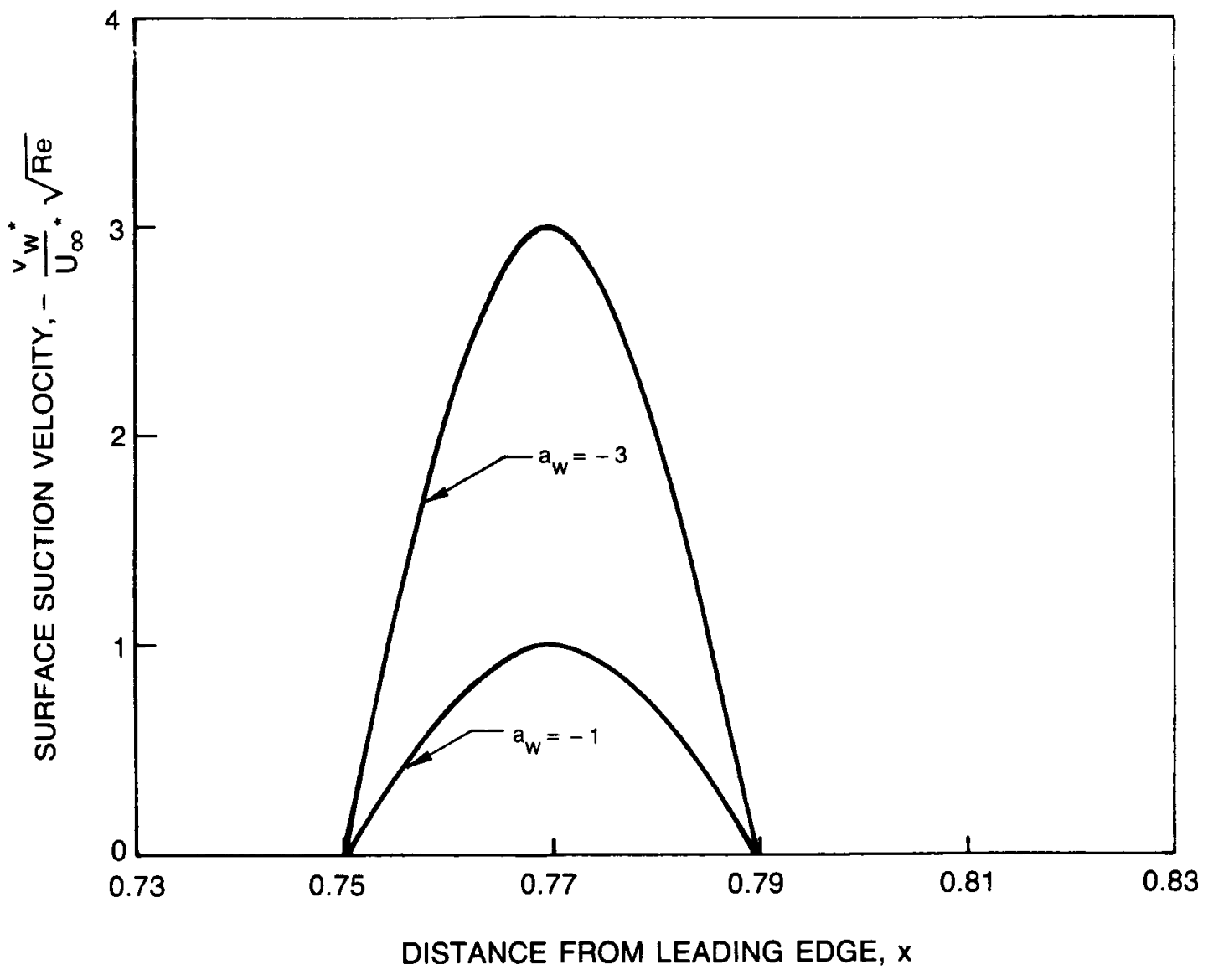

Fig. 19 Distribution of Slot Suction for NACA 0006 Airfoil 


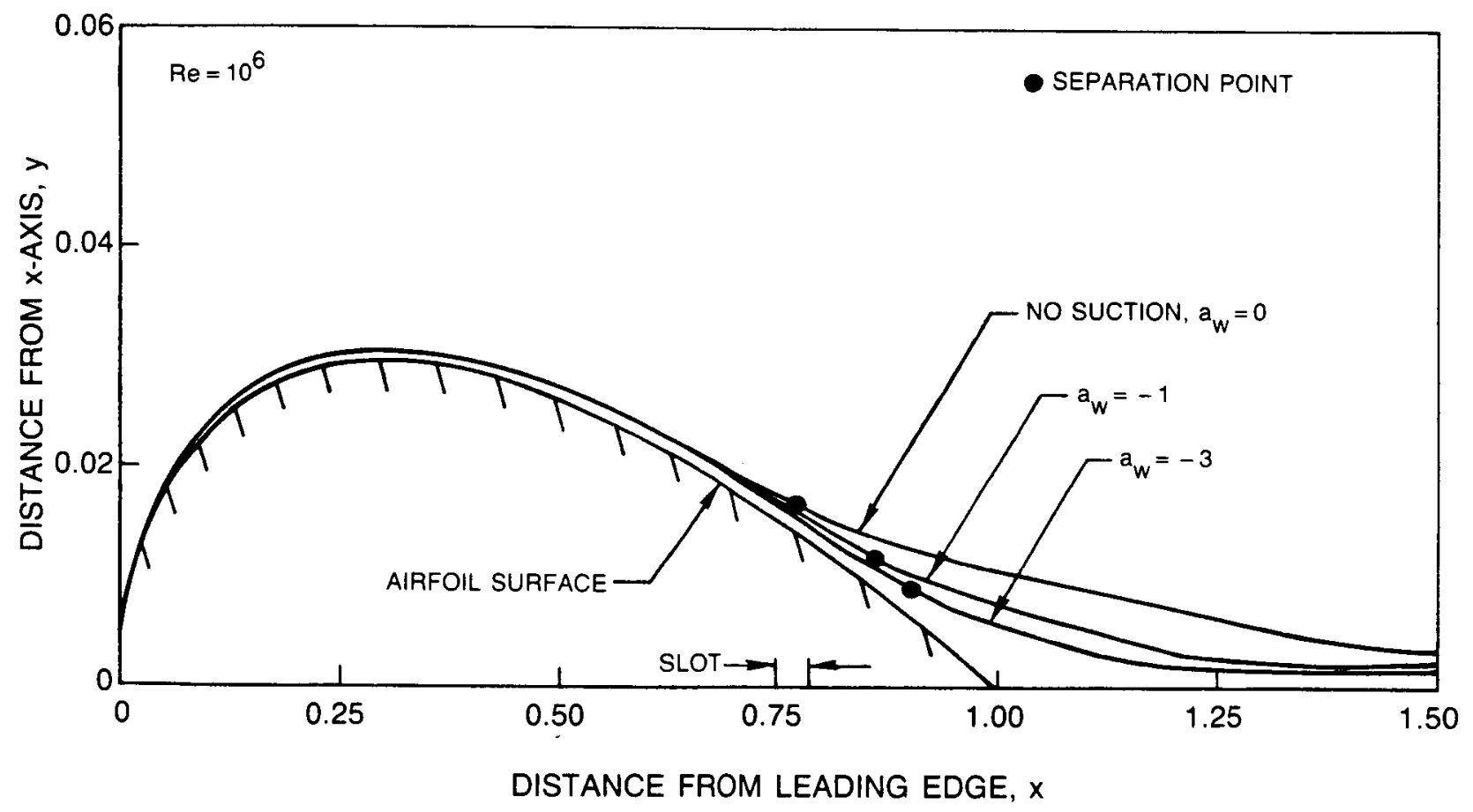

Fig. 20a Airfoil Profile and Displacement Body for NACA 0006 Airfoil with Slot Suction 


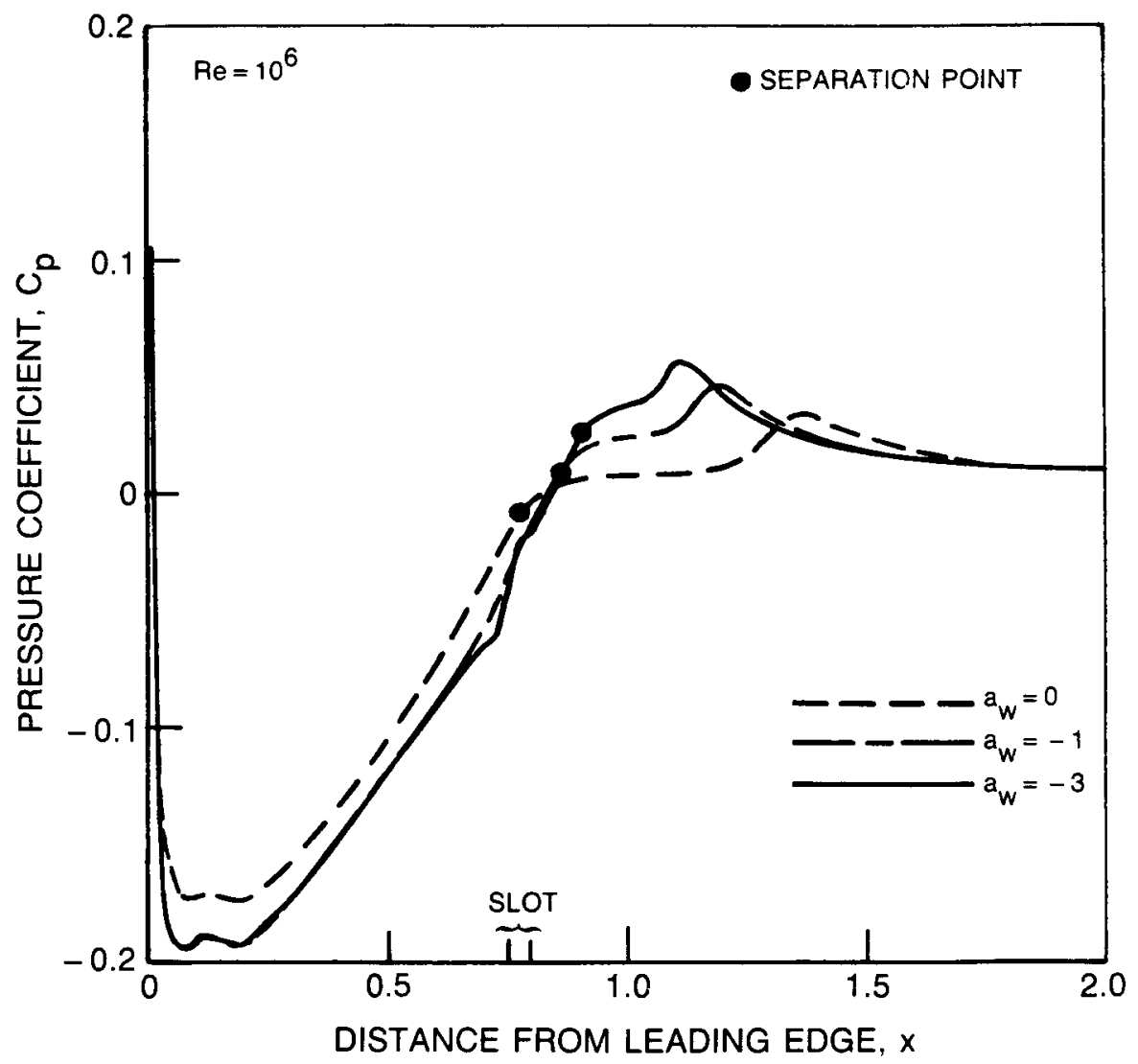

Fig. 20b Pressure Distribution for NACA 0006 Airfoil with Slot Suction 


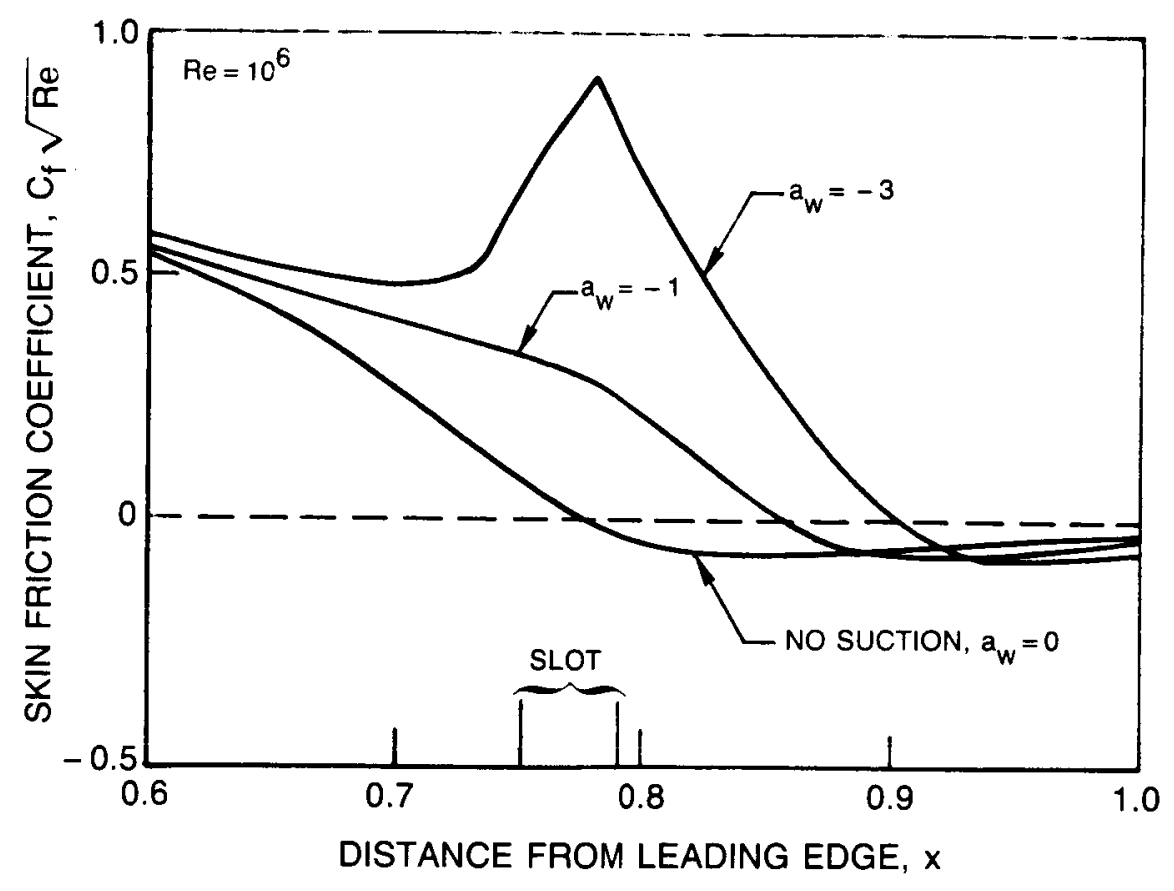

Fig. 20C Skin Friction Distribution in Trailing-Edge Region of NACA 0006 Airfoil with Slot Suction 


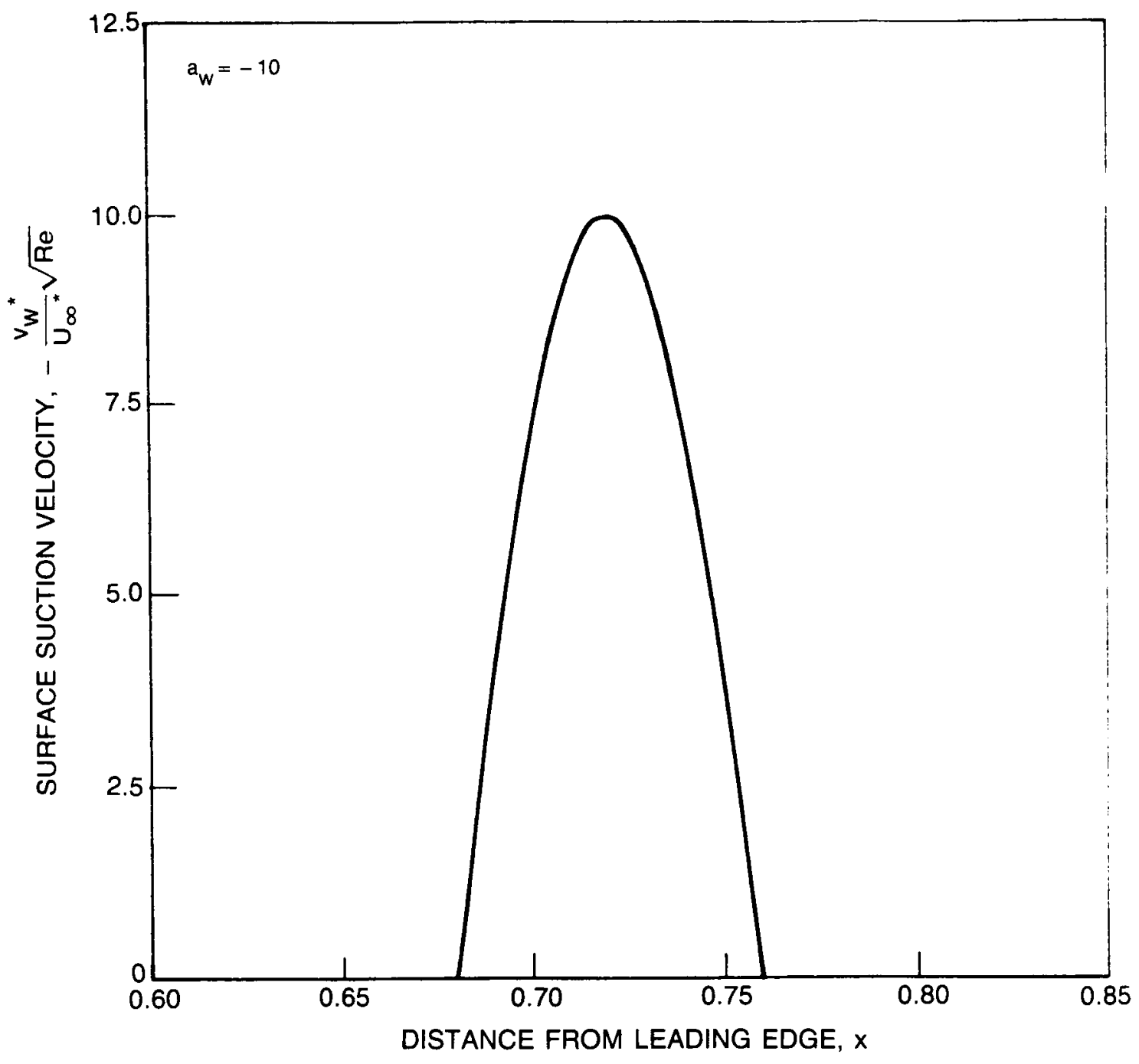

Fig. 21 Distribution of Slot Suction for NACA 0007 Airfoil 


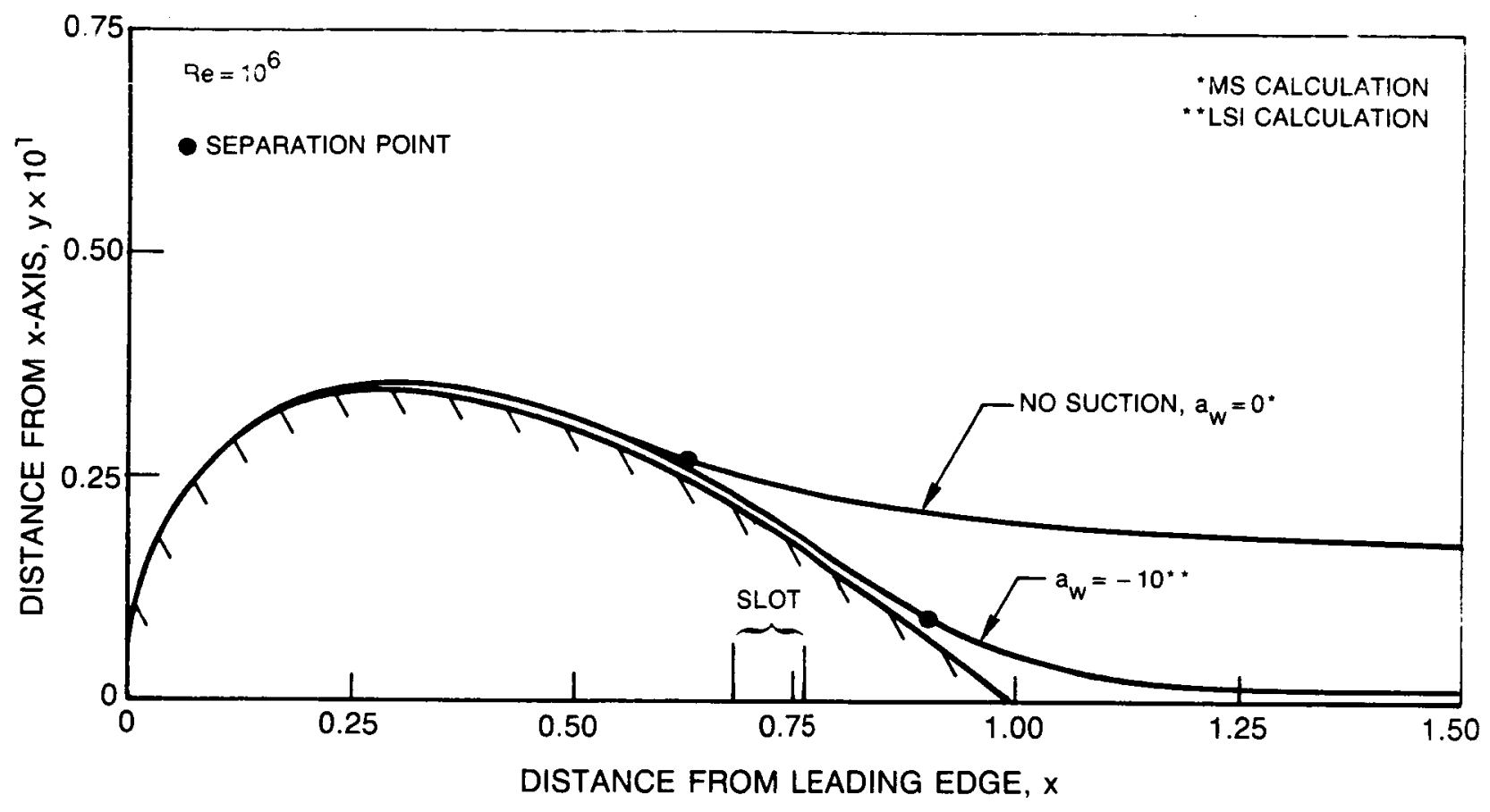

Fig. 22a Airfoil Profile and Displacement Body for NACA 0007 Airfoil with Slot Suction 


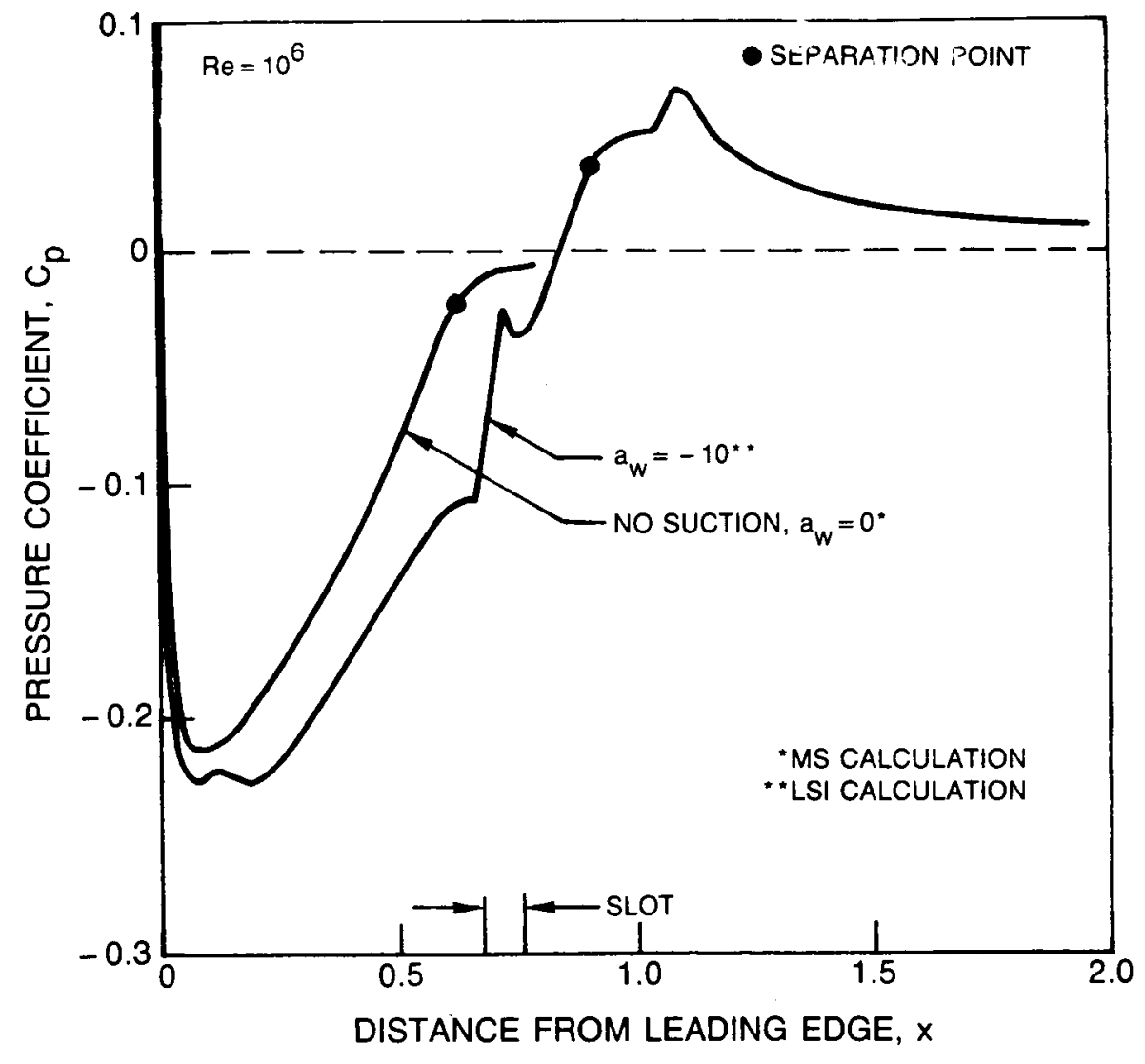

Fig. 22b Pressure Distribution for NACA 0007 Airfoil with Slot Suction 


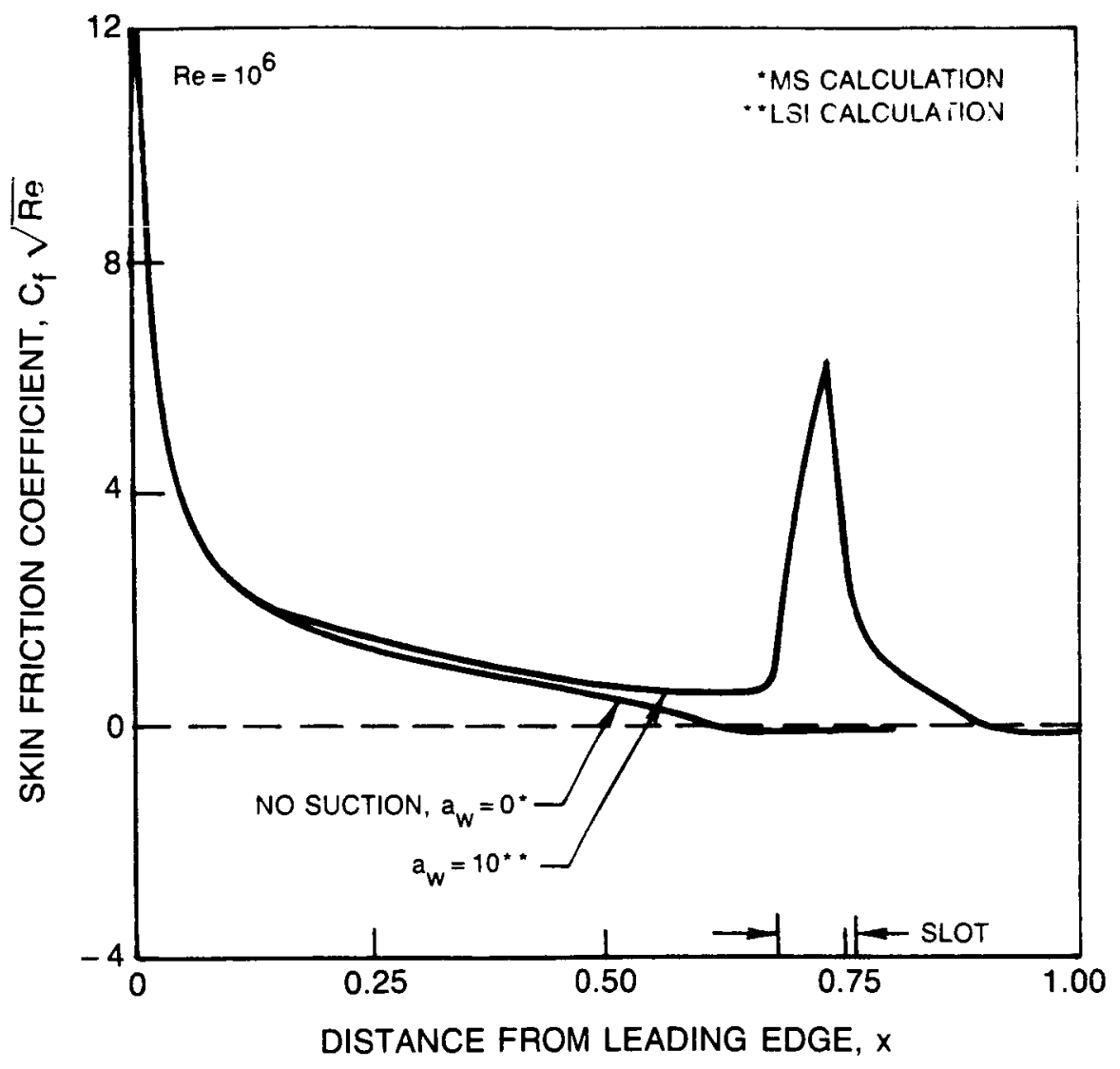

Fig. 22c Skin Friction Distribution for NACA 0007 Airfoil with Slot Suction 


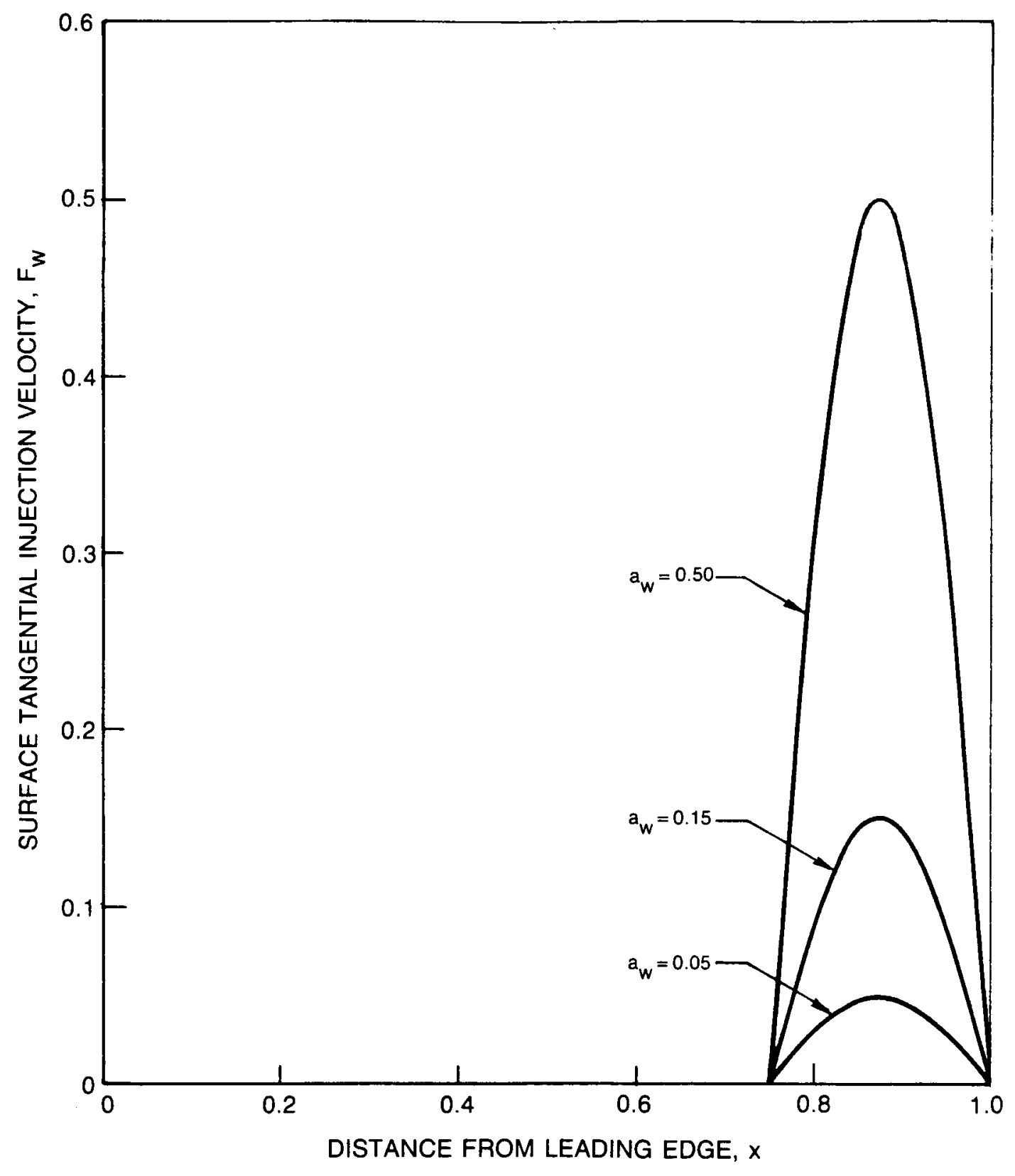

Fig. 23 Distribution of Tangential Injection Velocity for NACA 0006 Airfoil 


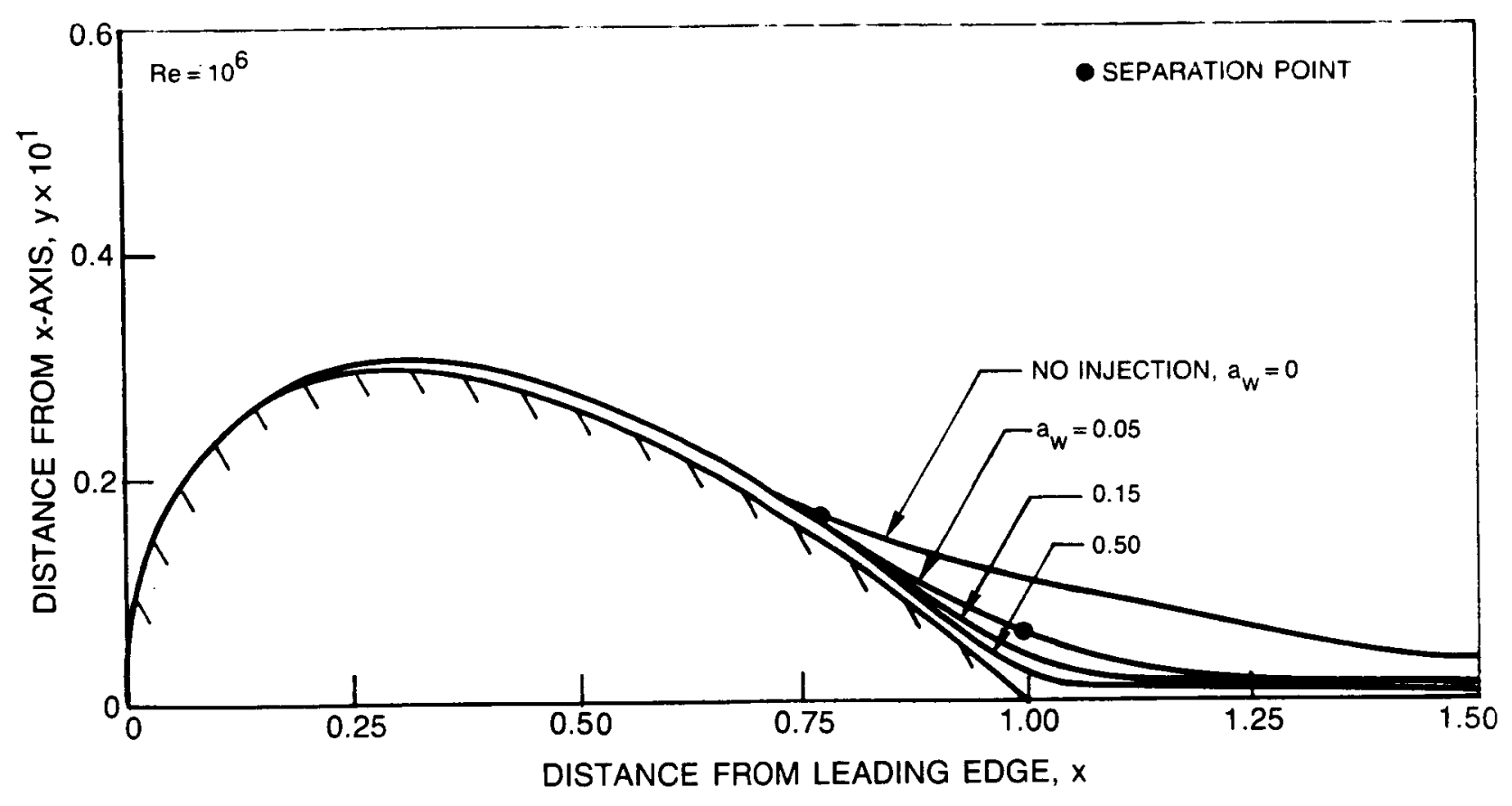

Fig. 24a Airfoil Profile and Displacement Body for NACA 0006 Airfoil with Tangential Injection 


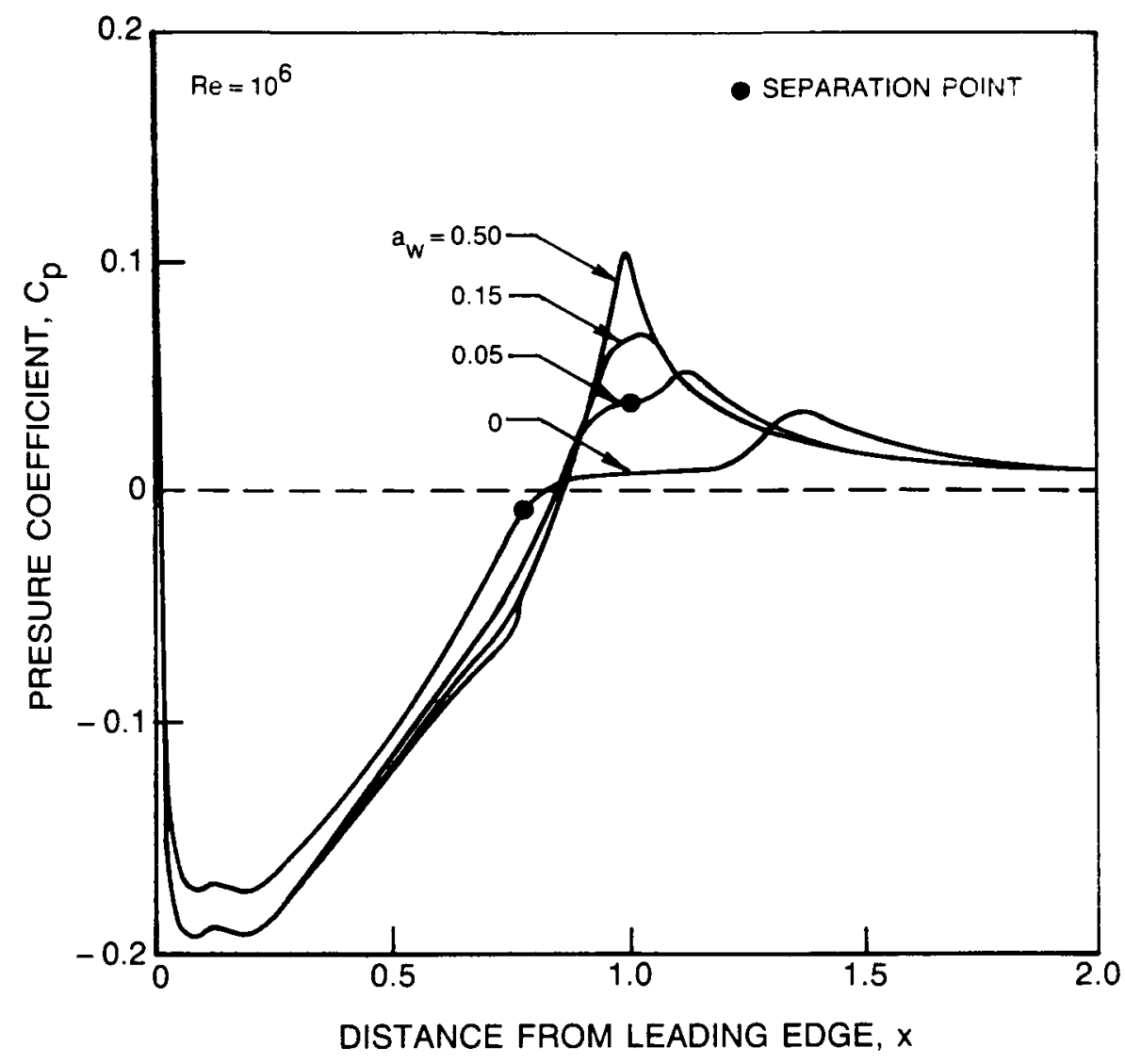

Fig. 24b Pressure Distribution for NACA 0006 Airfoil with Tangential Injection 


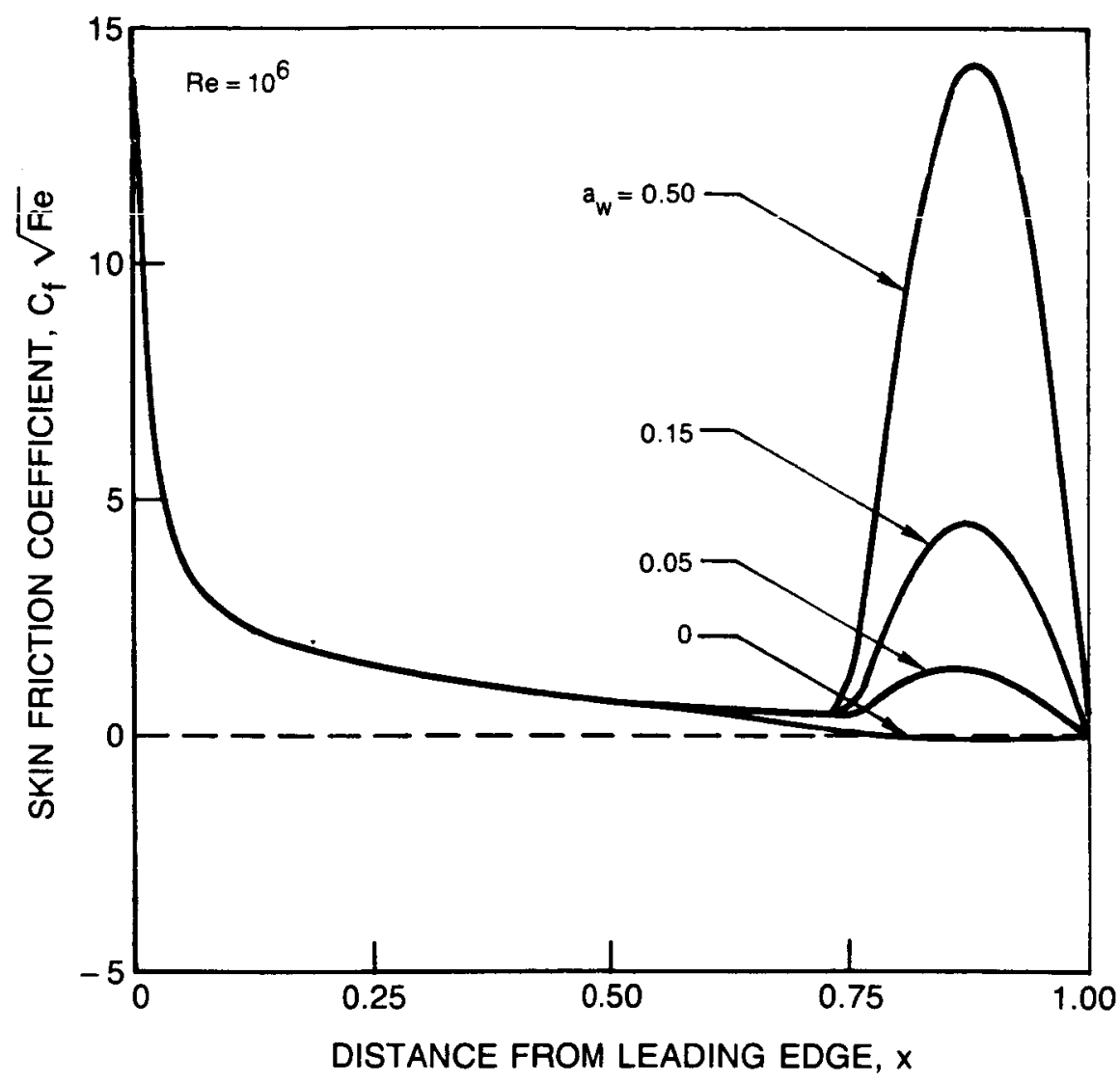

Fig. 24c Skin Friction Distribution for NACA 0006 Airfoil with Tangential Injection 
Standard Bibliographic Page

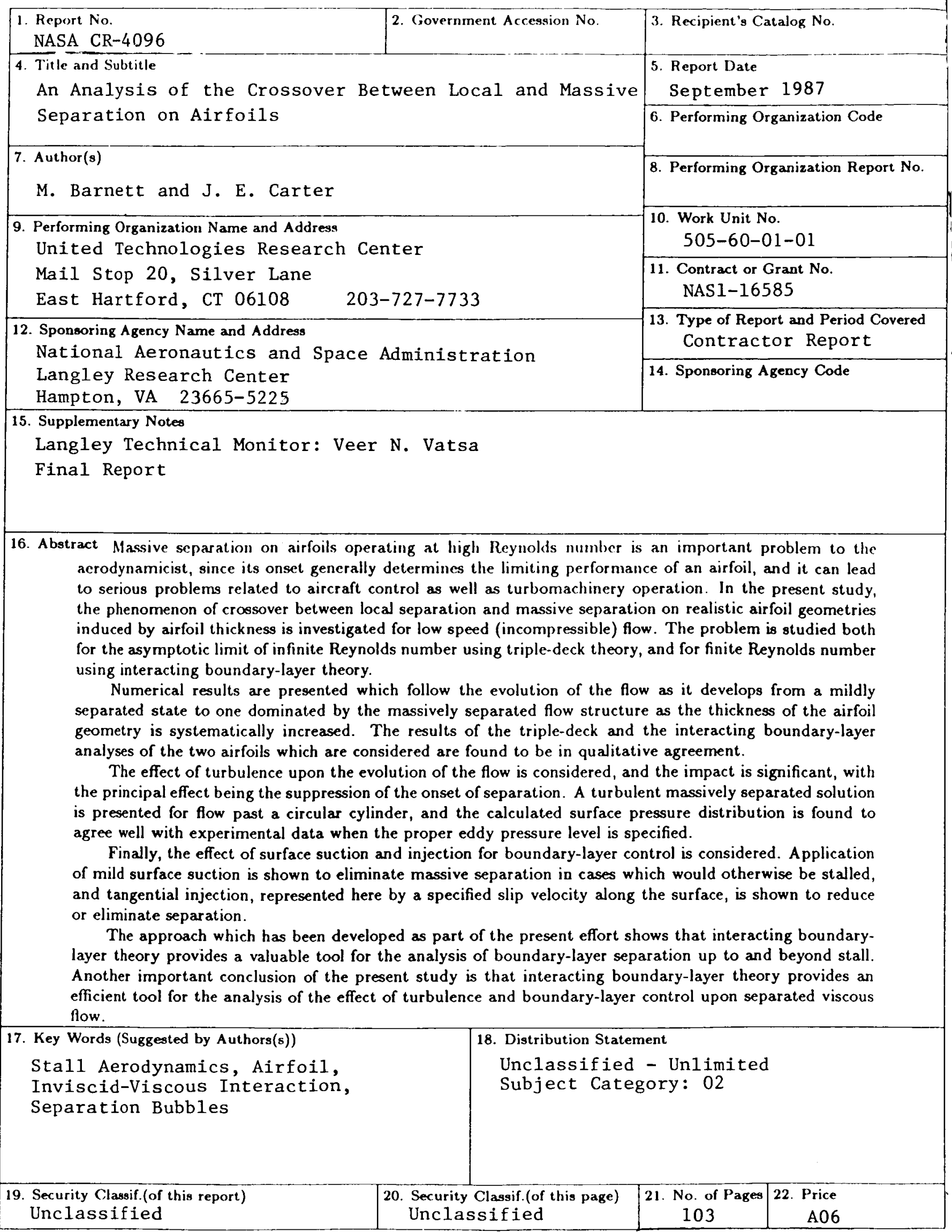

